\title{
Growth Behavior of Pineapple cv. Mauritius under Integrated Nutrient Management in Northern part of West Bengal, India
}

\author{
Nilesh Bhowmick ${ }^{1 *}$, Partha Sarathi Munsi², Swapan Kumar Ghosh ${ }^{1}$, \\ Prahlad Deb ${ }^{2}$ and Arunava Ghosh ${ }^{3}$
}

\author{
${ }^{1}$ Department of Pomology and Post-Harvest Technology, Uttar Banga Krishi Viswavidyalaya, \\ PO-Pundibari, Dist-Cooch Behar, West Bengal-736165, India \\ ${ }^{2}$ Department of Horticulture and Post-Harvest Technology, Institute of Agriculture, \\ Visva-Bharati, Sriniketan, West Bengal-731235, India \\ ${ }^{3}$ Discipline of Agricultural Statistics, Uttar Banga Krishi Viswavidyalaya, PO-Pundibari, \\ Dist-Cooch Behar, West Bengal-736165, India \\ *Corresponding author
}

\section{A B S T R A C T}

Pineapple is an important fruit crop and West Bengal is the leader in pineapple production in India. In West Bengal the commercial cultivars is Kew under Smooth Cayenne group

Keywords

Growth, Behavior, Pineapple, Nutrient, West Bengal.

Article Info

Accepted:

26 August 2017

Available Online:

10 September 2017 which suitable for processing purpose. Table purpose cultivars like Queen is being grown as home stead condition in some pockets of West Bengal. Mauritius cultivar (Queen Group) of pineapple was introduced first time in West Bengal from the south India and its growth performance under the integrated nutrient management practices was assessed in this present experiment. The experiment was conducted at farmers field near Bidhannagar area of Siliguri under the Darjeeling district from 2014-16. The experiment was conducted with Factorial Randomized block design having three factors- Chemical fertilizer (Factor A), organic manure (Factor B) and bio-fertilizer (Factor C) and eighteen treatment combination with three replication. It is found from the present experiment that the treatment combinations having chemical fertilizer, organic manure and bio-fertilizers shows better performance than the other treatment combinations. The plant height, canopy spread, number of leaves, D-leaf length, D-leaf breadth, and leaf area was recorded highest in $\mathrm{T}_{12}$ (Chemical $75 \% \mathrm{RDF}+$ Vermicompost + Bio-fertilizer).

\section{Introduction}

Pineapple (Ananas comosus L. Merr.) is an important tropical fruit of world under the Bromeliaceae family. Pineapple is one of the most internationalized fruit traded globally; it is third only to bananas and citrus in this respect. The major pineapple products are canned slices, chunks, crush, juice and fresh fruit. Processed pineapple products, such as juices, largely dominate this market, accounting for 80 percent of the trade (Jacob and Soman, 2006). India ranks $6^{\text {th }}(7.4 \%)$ in terms of world pineapple production (National Horticulture Database-2014) but productivity is quite low (15.8t/ha) compare to leading countries like Indonesia (124.5t/ha), Costa Rica (59.2t/ha), Brazil (40.9t/ha). West Bengal is leader in pineapple production in India (316 thousand metric 
tonnes). Pineapple is an important commercial fruit crop of West Bengal and it is intensively cultivated in Siliguri subdivision of Darjeeling district, Sadar subdivision of Jalpaiguri district, Islampur subdivision of Uttar Dinajpur district and parts of Cooch Behar district.

In West Bengal the commercial cultivars is Kew since long back. The Kew comes under Smooth Cayenne group suitable for processing purpose.

Table purpose cultivars like Queen is being grown as home stead condition in some pockets of West Bengal. Whereas, pineapple cv. Mauritius under the Queen group is very popular in southern parts of India (Kerala, Karnataka region) due to its taste, sweetness, flavor (Annon, 2017).

Considering the fact the Mauritius cultivar of pineapple was introduced first time in West Bengal from the south India and its performance under the integrated nutrient management practices was assessed in this present experiment.

\section{Materials and Methods}

The experiment was conducted at farmer's field near Bidhannagar area of Siliguri under the Darjeeling from 2014-16 with Spacing: $90 \mathrm{~cm} \times 35 \mathrm{~cm} \times 25 \mathrm{~cm}$ having individual bed size of: $3 \times 0.7 \mathrm{~m}=2.1 \mathrm{~m}^{2}$ with 25 number of plants per plot. The experiment was conducted with Asymmetrical Factorial Randomized Block design having three factors- Chemical fertilizer (Factor A), organic manure (Factor B) and bio-fertilizer (Factor C) and eighteen treatment combination with three replication. Chemical fertilizers were applied in 3 levels ( $\mathrm{A}_{0}$-zero, $\mathrm{A}_{1}-75$ percent and $\mathrm{A}_{2}-100$ percent recommended dose), organic manure were applied also in 3 levels ( $\mathrm{B}_{0}$-zero, $\mathrm{B}_{1}-\mathrm{FYM}$, $\mathrm{B}_{2}$-Vermicompost), bio-fertilizer were applied in 2 levels $\left(\mathrm{C}_{0}\right.$-zero and $\mathrm{C}_{1}$ - Azotobactor + Phosphate Solublising Bacteria). The doses for integrated nutrient management was as follows: Regular Dosages of Fertilizer $(R D F)=12: 4: 12$ g/plant, Farm Yard Manure $(\mathrm{FYM})=500 \mathrm{~g} /$ plant, Vermicompost $=$ $300 \mathrm{~g} /$ Plant, Azotobactor $=10 \mathrm{~g}$, Phosphate Solublising Bacteria (PSB) $=10 \mathrm{~g}$ was applied. It was reported that of $12 \mathrm{~g} /$ plants of nitrogen and potash has been found to be optimum and no effect of phosphorus was observed, however, $4 \mathrm{~g}$ of $\mathrm{P}_{2} \mathrm{O}_{5}$ increased fruit weight and yield in pineapple (Reddy and Prakash, 1982).

\section{Treatment combinations}

\begin{tabular}{|l|l|l|l|}
\hline $\mathrm{T}_{1}-$ & $\mathrm{A}_{0} \mathrm{~B}_{0} \mathrm{C}_{0}$ & $\mathrm{~T}_{10^{-}}$ & $\mathrm{A}_{1} \mathrm{~B}_{1} \mathrm{C}_{1}$ \\
\hline $\mathrm{T}_{2}-$ & $\mathrm{A}_{0} \mathrm{~B}_{0} \mathrm{C}_{1}$ & $\mathrm{~T}_{11^{-}}$ & $\mathrm{A}_{1} \mathrm{~B}_{2} \mathrm{C}_{0}$ \\
\hline $\mathrm{T}_{3}-$ & $\mathrm{A}_{0} \mathrm{~B}_{1} \mathrm{C}_{0}$ & $\mathrm{~T}_{12^{-}}$ & $\mathrm{A}_{1} \mathrm{~B}_{2} \mathrm{C}_{1}$ \\
\hline $\mathrm{T}_{4}{ }^{-}$ & $\mathrm{A}_{0} \mathrm{~B}_{1} \mathrm{C}_{1}$ & $\mathrm{~T}_{13}-$ & $\mathrm{A}_{2} \mathrm{~B}_{0} \mathrm{C}_{0}$ \\
\hline $\mathrm{T}_{5}-$ & $\mathrm{A}_{0} \mathrm{~B}_{2} \mathrm{C}_{0}$ & $\mathrm{~T}_{14^{-}}$ & $\mathrm{A}_{2} \mathrm{~B}_{0} \mathrm{C}_{1}$ \\
\hline $\mathrm{T}_{6}-$ & $\mathrm{A}_{0} \mathrm{~B}_{2} \mathrm{C}_{1}$ & $\mathrm{~T}_{15^{-}}$ & $\mathrm{A}_{2} \mathrm{~B}_{1} \mathrm{C}_{0}$ \\
\hline $\mathrm{T}_{7}-$ & $\mathrm{A}_{1} \mathrm{~B}_{0} \mathrm{C}_{0}$ & $\mathrm{~T}_{16^{-}}$ & $\mathrm{A}_{2} \mathrm{~B}_{1} \mathrm{C}_{1}$ \\
\hline $\mathrm{T}_{8}-$ & $\mathrm{A}_{1} \mathrm{~B}_{0} \mathrm{C}_{1}$ & $\mathrm{~T}_{17^{-}}$ & $\mathrm{A}_{2} \mathrm{~B}_{2} \mathrm{C}_{0}$ \\
\hline $\mathrm{T}_{9}-$ & $\mathrm{A}_{1} \mathrm{~B}_{1} \mathrm{C}_{0}$ & $\mathrm{~T}_{18^{-}}$ & $\mathrm{A}_{2} \mathrm{~B}_{2} \mathrm{C}_{1}$ \\
\hline
\end{tabular}

Regarding the growth behaviour the height of the plant $(\mathrm{cm})$, plant spread in North-South and East-West direction $(\mathrm{cm})$, number of leaves, D-leaf length $(\mathrm{cm})$, leaf length $(\mathrm{cm})$, leaf breadth $(\mathrm{cm})$, and leaf area $\left(\mathrm{cm}^{2}\right)$ were recorded 3 months after planting and continue upto 18 months with 3 months interval for 2015 and 2016. Analysis of variance for each parameter was performed using ProcGlm of Statistical Analysis System (SAS) software (version 9.3). Mean separation for different treatment under different parameter were performed using Least Significant Different (LSD) test $(\mathrm{P} \leq 0.05)$. Normality of residuals under the assumption of ANOVA was tested using Kolmogrov-Smirnov procedure using Proc-Univariate procedure of SAS (version 9.3). 


\section{Results and Discussion}

\section{Plant height (cm)}

The plant height of pineapple cv. Mauritius was varied significant among different treatments and it was found highest in $\mathrm{T}_{10}$ and $\mathrm{T}_{12}$ for 2015 and 2016 at 3 months after planting. From 9 months after planting to 18 months after planting the maximum plant height was recorded in $\mathrm{T}_{12}$ for 2015, 2016 and for pooled values. The height of pineapple plant was $83.71 \mathrm{~cm}$ (pooled) and $90.76 \mathrm{~cm}$ (pooled) for 15 and 18 months after planting in $\mathrm{T}_{12}$ followed by $79.96 \mathrm{~cm}$ (pooled) and $87.53 \mathrm{~cm}$ (pooled) in $\mathrm{T}_{10}$, respectively. The result from table 1 show clearly there is significant role of nutrient management for increasing the height of pineapple plants. Comparing the $T_{11}$ and $T_{12}$ it is clear that there is a great role of bio-fertilizer for the growth of pineapple plants.

\section{Plant canopy spread (cm)}

Observation revealed (Tables 2 and 3) that the plant canopy on North-South and East-West direction was increased in all the treatments from 3 months after planting up to the 18 months after planting. Significant variation between the main factor and treatments combination with respect to canopy spread was observed among several nutrient treatments.

Treatments combination $\mathrm{T}_{10}, \mathrm{~T}_{11}, \mathrm{~T}_{12}, \mathrm{~T}_{18}$ shows the better performance compare with other treatment combinations. For all observation months the lowest canopy spread was recorded with $\mathrm{T}_{1}$ (no nutrient). The performance of canopy spread was better in treatments where chemical, organic and biofertilizers were applied combined compared with sole application of bio-fertilizers, or organic or only chemical fertilizers. The rate of increase of canopy was higher from 3 months to 6 months and 12 months to 15 months after planting for both north-south and east- west direction. Maximum canopy spread in north-south and east-west direction was observed with $\mathrm{T}_{12}$ for 2015, 2016 and polled values. The spread was $117.64 \mathrm{~cm}$ and $124.20 \mathrm{~cm}$ respectively for north-south and east-west direction at 18 months after planting.

\section{Number of leaves}

The number of leaves of pineapple increased from three months to eighteen months and it varied significantly mostly among all the treatments. Flower induction is an important practice in pineapple normally done during 11-12 months after planting and for effectiveness the number of leaves in pineapple has an important factor and 30-40 leaves are required for flowering of pineapple. At 12 months after planting, the number of leaves was 49.47 and 49.27, respectively with $\mathrm{T}_{12}$. Lowest number (pooled mean) of leaves (36.14 and 39.27) was observed with $\mathrm{T}_{1}$ (no nutrient) and maximum number (pooled mean) of leaves (55.75 and 58.87) were recorded with $T_{12}$ which was statistically at par with $\mathrm{T}_{9}, \mathrm{~T}_{10}, \mathrm{~T}_{11}, \mathrm{~T}_{14}, \mathrm{~T}_{16}, \mathrm{~T}_{18}$, respectively at 15 and 18 months after planting, respectively. The rate of increase of number of leaves was higher from 9 to 12 months after planting. Treatments with having all the chemicals, organic and bio-fertilizers combinations showed better performance compare with individual effect of organic, chemical and bio-fertilizers.

\section{D-leaf length, breadth and area}

D-leaf is the most physiological active leaves of pineapple and it is highly co-related to growth behaviour, nutrient content of pineapple leaves. Leaf size is an important parameter for most of the crop regarding the flowering, fruiting and subsequent yield. 
Table.1 Effect of Nutrient management on plant height $(\mathrm{cm})$

\begin{tabular}{|c|c|c|c|c|c|c|c|c|c|}
\hline \multirow[t]{2}{*}{ Treatments } & \multicolumn{3}{|c|}{3 MAP } & \multicolumn{3}{|c|}{6 MAP } & \multicolumn{3}{|c|}{9 MAP } \\
\hline & 2015 & 2016 & Mean & 2015 & 2016 & Mean & 2015 & 2016 & Mean \\
\hline $\mathrm{A}_{0}$ & $23.95 \mathrm{c}$ & $23.97 \mathrm{c}$ & $23.96 \mathrm{c}$ & $30.1 \mathrm{c}$ & $32.36 \mathrm{c}$ & $31.23 \mathrm{a}$ & $37.96 \mathrm{~b}$ & $38.16 \mathrm{~b}$ & $38.06 \mathrm{~b}$ \\
\hline $\mathrm{A}_{1}$ & $32.64 \mathrm{a}$ & $33.13 \mathrm{a}$ & $32.89 \mathrm{a}$ & $40.08 \mathrm{a}$ & $42.40 \mathrm{a}$ & $41.24 \mathrm{a}$ & $53.85 \mathrm{a}$ & $54.8 \mathrm{a}$ & $54.33 \mathrm{a}$ \\
\hline $\mathrm{A}_{2}$ & $31.29 \mathrm{~b}$ & $31.88 \mathrm{~b}$ & $31.59 \mathrm{~b}$ & $39.16 \mathrm{~b}$ & $41.28 \mathrm{~b}$ & $40.22 b$ & $50.61 \mathrm{a}$ & $50.73 a$ & $50.67 \mathrm{~b}$ \\
\hline S.Em. ( \pm$)$ & 0.28 & 0.29 & 0.26 & 0.24 & 0.27 & 0.23 & 1.67 & 1.69 & 1.68 \\
\hline L.S.D(P $\leq 0.05)$ & 0.81 & 0.85 & 0.76 & 0.70 & 0.76 & 0.67 & 4.80 & 4.85 & 4.82 \\
\hline $\mathrm{B}_{0}$ & $28.49 \mathrm{~b}$ & $28.72 \mathrm{~b}$ & $28.61 \mathrm{~b}$ & $34.68 \mathrm{~b}$ & $36.81 \mathrm{~b}$ & $35.75 \mathrm{~b}$ & $43.52 \mathrm{~b}$ & $44.30 \mathrm{~b}$ & $43.91 \mathrm{~b}$ \\
\hline $\mathrm{B}_{1}$ & $29.64 \mathrm{a}$ & $29.92 \mathrm{a}$ & $29.78 \mathrm{a}$ & $37.07 \mathrm{a}$ & $39.30 \mathrm{a}$ & $38.19 \mathrm{a}$ & $48.84 \mathrm{a}$ & $49.02 \mathrm{ab}$ & $48.93 a$ \\
\hline $\mathrm{B}_{2}$ & $25.75 a$ & $30.34 a$ & $30.05 \mathrm{a}$ & $37.59 \mathrm{a}$ & $39.92 a$ & $38.76 \mathrm{a}$ & $50.06 a$ & $50.37 \mathrm{a}$ & $50.22 \mathrm{a}$ \\
\hline S.Em. ( \pm$)$ & 0.28 & 0.29 & 0.26 & 0.24 & 0.27 & 0.23 & 1.67 & 1.69 & 1.68 \\
\hline L.S.D $(\mathrm{P} \leq 0.05)$ & 0.81 & 0.85 & 0.76 & 0.70 & 0.76 & 0.67 & 4.80 & 4.85 & 4.82 \\
\hline $\mathrm{C}_{0}$ & $28.08 \mathrm{~b}$ & $28.26 \mathrm{~b}$ & $28.18 b$ & $34.66 \mathrm{~b}$ & $36.89 \mathrm{~b}$ & $35.78 \mathrm{~b}$ & $44.59 \mathrm{~b}$ & $45.00 \mathrm{~b}$ & $44.79 \mathrm{~b}$ \\
\hline $\mathrm{C}_{1}$ & $30.50 \mathrm{a}$ & $31.06 \mathrm{a}$ & $30.78 \mathrm{a}$ & $38.24 \mathrm{a}$ & $40.46 \mathrm{a}$ & $38.36 \mathrm{a}$ & $50.36 \mathrm{a}$ & $50.80 \mathrm{a}$ & $50.58 \mathrm{a}$ \\
\hline S.Em. $( \pm)$ & 0.23 & 0.24 & 0.21 & 0.2 & 0.22 & 0.19 & 1.36 & 1.38 & 1.37 \\
\hline L.S.D(P $\leq 0.05)$ & 0.66 & 0.69 & 0.62 & 0.57 & 0.62 & 0.54 & 3.92 & 3.96 & 3.93 \\
\hline
\end{tabular}

\begin{tabular}{|c|c|c|c|c|c|c|c|c|c|c|}
\hline \multirow{2}{*}{\multicolumn{2}{|c|}{$\begin{array}{l}\text { Treatments/ } \\
\text { Combination }\end{array}$}} & \multicolumn{3}{|c|}{3 MAP } & \multicolumn{3}{|c|}{6 MAP } & \multicolumn{3}{|c|}{9 MAP } \\
\hline & & 2015 & 2016 & Mean & 2015 & 2016 & Mean & 2015 & 2016 & Mean \\
\hline $\mathrm{T}_{1}$ & $\mathrm{~A}_{0} \mathrm{~B}_{0} \mathrm{C}_{0}$ & $21.02 \mathrm{f}$ & $21.01 \mathrm{~g}$ & $21.02 \mathrm{~g}$ & $26.19 \mathrm{~h}$ & $28.84 \mathrm{~g}$ & $27.52 \mathrm{~h}$ & $31.91 \mathrm{~g}$ & $32.5 \mathrm{~g}$ & $32.21 \mathrm{~g}$ \\
\hline$T_{2}$ & $\mathrm{~A}_{0} \mathrm{~B}_{0} \mathrm{C}_{1}$ & $25.63 \mathrm{~d}$ & $25.39 \mathrm{f}$ & $25.51 \mathrm{e}$ & $31.86 \mathrm{f}$ & $33.69 \mathrm{e}$ & $32.78 \mathrm{f}$ & $38.61 \mathrm{efg}$ & $40.03 \mathrm{efg}$ & $39.32 \mathrm{efg}$ \\
\hline $\mathrm{T}_{3}$ & $\mathrm{~A}_{0} \mathrm{~B}_{1} \mathrm{C}_{0}$ & $21.51 \mathrm{ef}$ & $22.03 \mathrm{~g}$ & $21.77 \mathrm{fg}$ & $28.18 \mathrm{~g}$ & $30.38 \mathrm{fg}$ & $29.28 \mathrm{~g}$ & $35.75 \mathrm{fg}$ & $36.33 \mathrm{fg}$ & $36.04 \mathrm{fg}$ \\
\hline $\mathrm{T}_{4}$ & $\mathrm{~A}_{0} \mathrm{~B}_{1} \mathrm{C}_{1}$ & $26.37 \mathrm{~d}$ & $26.09 \mathrm{f}$ & $26.23 \mathrm{e}$ & $32.83 \mathrm{f}$ & $34.66 \mathrm{e}$ & $33.75 \mathrm{f}$ & 40.95defg & $40.13 \mathrm{efg}$ & 40.54efg \\
\hline $\mathrm{T}_{5}$ & $\mathrm{~A}_{0} \mathrm{~B}_{2} \mathrm{C}_{0}$ & $23.02 \mathrm{e}$ & $22.97 \mathrm{~g}$ & $23.00 \mathrm{f}$ & $29.44 \mathrm{~g}$ & $31.09 \mathrm{f}$ & $30.27 \mathrm{~g}$ & $38.85 \mathrm{efg}$ & $38.47 \mathrm{efg}$ & $38.66 \mathrm{efg}$ \\
\hline $\mathrm{T}_{6}$ & $\mathrm{~A}_{0} \mathrm{~B}_{2} \mathrm{C}_{1}$ & $26.17 \mathrm{~d}$ & $26.3 \mathrm{f}$ & $26.24 \mathrm{e}$ & $32.10 \mathrm{f}$ & $35.51 \mathrm{e}$ & $33.81 \mathrm{f}$ & 41.72defg & 41.5defg & 41.61defg \\
\hline $\mathrm{T}_{7}$ & $\mathrm{~A}_{1} \mathrm{~B}_{0} \mathrm{C}_{0}$ & $30.34 \mathrm{c}$ & $31.06 \mathrm{cde}$ & $30.70 \mathrm{~cd}$ & $36.53 \mathrm{de}$ & $38.97 \mathrm{~cd}$ & $37.75 \mathrm{de}$ & $45.97 \mathrm{cdef}$ & $46.7 \mathrm{cdef}$ & 46.34cdef \\
\hline $\mathrm{T}_{8}$ & $\mathrm{~A}_{1} \mathrm{~B}_{0} \mathrm{C}_{1}$ & $31.59 \mathrm{bc}$ & $32.34 \mathrm{bcd}$ & $31.97 \mathrm{bc}$ & $38.69 b c$ & $40.53 \mathrm{bc}$ & 39.61be & 49.28bcde & 49.97bcde & 49.63bcde \\
\hline $\mathrm{T}_{9}$ & $\mathrm{~A}_{1} \mathrm{~B}_{1} \mathrm{C}_{0}$ & $33.34 \mathrm{ab}$ & $33.00 \mathrm{abc}$ & $33.17 \mathrm{ab}$ & $38.65 \mathrm{bc}$ & $41.32 \mathrm{~b}$ & $39.99 b c$ & 52.06abcd & 52.67abcd & 52.37abcd \\
\hline $\mathrm{T}_{10}$ & $\mathrm{~A}_{1} \mathrm{~B}_{1} \mathrm{C}_{1}$ & $33.86 a$ & $34.32 \mathrm{ab}$ & $34.09 \mathrm{a}$ & $42.41 \mathrm{a}$ & $44.68 \mathrm{a}$ & $43.55 \mathrm{a}$ & $58.57 \mathrm{ab}$ & $59.73 \mathrm{ab}$ & $59.15 \mathrm{ab}$ \\
\hline $\mathrm{T}_{11}$ & $\mathrm{~A}_{1} \mathrm{~B}_{2} \mathrm{C}_{0}$ & $32.61 \mathrm{a}$ & $33.51 \mathrm{ab}$ & $33.06 \mathrm{ab}$ & $41.74 \mathrm{a}$ & $43.88 \mathrm{a}$ & $42.81 \mathrm{a}$ & $53.99 \mathrm{abc}$ & $55.47 \mathrm{abc}$ & $54.73 \mathrm{abc}$ \\
\hline $\mathrm{T}_{12}$ & $\mathrm{~A}_{1} \mathrm{~B}_{2} \mathrm{C}_{1}$ & $33.07 \mathrm{ab}$ & $34.56 \mathrm{a}$ & $33.82 \mathrm{ab}$ & $42.48 \mathrm{a}$ & $44.99 \mathrm{a}$ & $43.74 a$ & $63.20 \mathrm{a}$ & $64.27 \mathrm{a}$ & $63.74 a$ \\
\hline $\mathrm{T}_{13}$ & $\mathrm{~A}_{2} \mathrm{~B}_{0} \mathrm{C}_{0}$ & $29.94 c$ & $29.76 \mathrm{e}$ & $29.85 d$ & $35.61 \mathrm{e}$ & $37.7 \mathrm{~d}$ & $36.66 \mathrm{e}$ & $45.57 \mathrm{cdef}$ & $46.53 \mathrm{cdef}$ & $46.05 \mathrm{cdef}$ \\
\hline $\mathrm{T}_{14}$ & $\mathrm{~A}_{2} \mathrm{~B}_{0} \mathrm{C}_{1}$ & $32.39 \mathrm{ab}$ & $32.77 \mathrm{abc}$ & $32.58 \mathrm{ab}$ & $39.19 \mathrm{~b}$ & $41.14 \mathrm{~b}$ & $40.17 b$ & 49.81bcde & $50.07 \mathrm{bcde}$ & 49.94bcde \\
\hline $\mathrm{T}_{15}$ & $\mathrm{~A}_{2} \mathrm{~B}_{1} \mathrm{C}_{0}$ & $30.10 \mathrm{c}$ & $30.38 \mathrm{de}$ & $30.24 \mathrm{~cd}$ & $38.13 \mathrm{bcd}$ & $40.34 \mathrm{bc}$ & $39.24 \mathrm{bcd}$ & 49.71bdde & 49.53bdce & $49.62 \mathrm{bcde}$ \\
\hline $\mathrm{T}_{16}$ & $\mathrm{~A}_{2} \mathrm{~B}_{1} \mathrm{C}_{1}$ & $32.67 \mathrm{ab}$ & $33.68 \mathrm{ab}$ & $33.18 \mathrm{ab}$ & $42.25 \mathrm{a}$ & $44.45 \mathrm{a}$ & $43.35 \mathrm{a}$ & $55.98 \mathrm{abc}$ & $55.7 \mathrm{abc}$ & $55.84 \mathrm{abc}$ \\
\hline $\mathrm{T}_{17}$ & $\mathrm{~A}_{2} \mathrm{~B}_{2} \mathrm{C}_{0}$ & $29.86 c$ & 30.66de & $30.26 \mathrm{~cd}$ & $37.45 \mathrm{~cd}$ & $39.52 \mathrm{bcd}$ & $38.49 \mathrm{~cd}$ & 47.49bcdef & 46.77cdef & $47.13 \mathrm{cdef}$ \\
\hline $\mathrm{T}_{18}$ & $\mathrm{~A}_{2} \mathrm{~B}_{2} \mathrm{C}_{1}$ & $32.75 \mathrm{ab}$ & $34.05 \mathrm{ab}$ & $33.40 \mathrm{ab}$ & $42.33 \mathrm{a}$ & $44.53 \mathrm{a}$ & $43.43 \mathrm{a}$ & $55.08 \mathrm{abc}$ & 55.77abc & $55.43 \mathrm{abc}$ \\
\hline \multicolumn{2}{|c|}{ S.Em. $( \pm)$} & 0.69 & 0.72 & 0.64 & 0.6 & 0.65 & 0.57 & 4.09 & 4.14 & 4.11 \\
\hline \multicolumn{2}{|c|}{ L.S.D. $(\mathrm{P} \leq 0.05)$} & 1.98 & 2.07 & 1.85 & 1.72 & 1.87 & 1.63 & 11.76 & 11.89 & 11.80 \\
\hline
\end{tabular}

MAP-Month after planting. **Means with the same letter are not significantly different 
Table.1 Effect of Nutrient management on plant height (cm) (contd....)

\begin{tabular}{|c|c|c|c|c|c|c|c|c|c|}
\hline \multirow[t]{2}{*}{ Treatments } & \multicolumn{3}{|c|}{12 MAP } & \multicolumn{3}{|c|}{15 MAP } & \multicolumn{3}{|c|}{18 MAP } \\
\hline & 2015 & 2016 & Mean & 2015 & 2016 & Mean & 2015 & 2016 & Mean \\
\hline$\overline{A_{0}}$ & $52.45 b$ & $54.39 \mathrm{~b}$ & $53.39 \mathrm{~b}$ & $60.64 b$ & $63.95 \mathrm{~b}$ & $62.30 \mathrm{~b}$ & $68.79 \mathrm{~b}$ & $69.71 b$ & $69.25 b$ \\
\hline $\mathrm{A}_{1}$ & $66.37 \mathrm{a}$ & $66.59 \mathrm{a}$ & $66.49 a$ & $76.10 \mathrm{a}$ & $74.69 \mathrm{a}$ & $75.40 \mathrm{a}$ & $81.11 \mathrm{a}$ & $81.89 \mathrm{a}$ & $81.50 \mathrm{a}$ \\
\hline $\mathrm{A}_{2}$ & $65.81 \mathrm{a}$ & $63.78 \mathrm{a}$ & $64.80 \mathrm{a}$ & $72.89 \mathrm{a}$ & $72.04 a$ & $72.47 \mathrm{a}$ & $77.85 \mathrm{a}$ & $78.39 \mathrm{a}$ & $78.12 a$ \\
\hline S.Em. ( \pm$)$ & 1.78 & 2.01 & 1.77 & 1.68 & 2.09 & 1.85 & 1.58 & 1.79 & 1.57 \\
\hline L.S.D(P $\leq 0.05)$ & 5.12 & 5.78 & 5.09 & 4.84 & 6.00 & 5.32 & 4.54 & 5.13 & 4.50 \\
\hline $\mathrm{B}_{0}$ & $58.93 \mathrm{a}$ & $57.32 \mathrm{~b}$ & $58.12 \mathrm{~b}$ & $66.19 \mathrm{~b}$ & $66.71 \mathrm{~b}$ & $66.45 \mathrm{~b}$ & $71.83 \mathrm{~b}$ & $72.62 \mathrm{~b}$ & $77.23 \mathrm{~b}$ \\
\hline $\mathrm{B}_{1}$ & $61.85 \mathrm{a}$ & $62.74 \mathrm{~b}$ & $62.30 \mathrm{ab}$ & $70.93 b$ & $70.83 b$ & $70.88 \mathrm{ab}$ & $77.25 \mathrm{a}$ & $77.69 \mathrm{ab}$ & $77.49 \mathrm{a}$ \\
\hline $\mathrm{B}_{2}$ & $63.85 \mathrm{a}$ & $64.65 a$ & $64.25 \mathrm{a}$ & $72.51 \mathrm{a}$ & $73.16 \mathrm{a}$ & $72.84 a$ & $78.67 \mathrm{a}$ & $79.68 \mathrm{a}$ & $79.18 \mathrm{a}$ \\
\hline S.Em. ( \pm$)$ & 1.78 & 2.01 & 1.77 & 1.68 & 2.09 & 1.85 & 1.58 & 1.79 & 1.57 \\
\hline L.S.D $(P \leq 0.05)$ & 5.12 & 5.78 & 5.09 & 4.84 & 6.00 & 5.32 & 4.54 & 5.13 & 4.50 \\
\hline $\mathrm{C}_{0}$ & $59.17 \mathrm{~b}$ & $58.89 \mathrm{~b}$ & $59.03 \mathrm{~b}$ & $67.15 b$ & $67.31 \mathrm{~b}$ & $67.23 \mathrm{~b}$ & $73.30 \mathrm{~b}$ & $73.40 \mathrm{~b}$ & $79.35 b$ \\
\hline $\mathrm{C}_{1}$ & $63.91 \mathrm{a}$ & $64.25 \mathrm{a}$ & $64.08 \mathrm{a}$ & $72.60 \mathrm{a}$ & $73.15 \mathrm{a}$ & $72.88 \mathrm{a}$ & $78.53 a$ & $79.93 a$ & $79.23 \mathrm{a}$ \\
\hline S.Em. ( \pm$)$ & 1.45 & 1.64 & 1.45 & 1.38 & 1.71 & 1.51 & 1.29 & 1.46 & 1.28 \\
\hline L.S.D(P $\leq 0.05)$ & 4.18 & 4.72 & 4.15 & 3.95 & 4.90 & 4.34 & 3.71 & 4.19 & 3.67 \\
\hline
\end{tabular}

\begin{tabular}{|c|c|c|c|c|c|c|c|c|c|c|}
\hline \multirow{2}{*}{\multicolumn{2}{|c|}{$\begin{array}{l}\text { Treatments/ } \\
\text { Combination }\end{array}$}} & \multicolumn{3}{|c|}{12 MAP } & \multicolumn{3}{|c|}{15 MAP } & \multicolumn{3}{|c|}{18 MAP } \\
\hline & & \multirow{2}{*}{$\begin{array}{c}\mathbf{2 0 1 5} \\
43.25 \mathrm{f} \\
\end{array}$} & \multirow{2}{*}{$\begin{array}{l}\mathbf{2 0 1 6} \\
45.5 \mathrm{e}^{2}\end{array}$} & \multirow{2}{*}{$\begin{array}{l}\text { Mean } \\
44.38 \mathrm{f} \\
\end{array}$} & \multirow{2}{*}{$\begin{array}{r}\mathbf{2 0 1 5} \\
55.43 \mathrm{f} \\
\end{array}$} & \multirow{2}{*}{$\begin{array}{l}\mathbf{2 0 1 6} \\
57 \mathrm{~d}\end{array}$} & \multirow{2}{*}{$\begin{array}{c}\text { Mean } \\
56.22 \mathrm{f} \\
\end{array}$} & \multirow{2}{*}{$\begin{array}{r}\mathbf{2 0 1 5} \\
63.08 \mathrm{f} \\
\end{array}$} & \multirow{2}{*}{\begin{tabular}{|l|}
$\mathbf{2 0 1 6}$ \\
$62.60 \mathrm{f}$
\end{tabular}} & \multirow{2}{*}{$\begin{array}{l}\text { Mean } \\
62.84 \mathrm{f} \\
\end{array}$} \\
\hline $\mathrm{T}_{1}$ & $\mathrm{~A}_{0} \mathrm{~B}_{0} \mathrm{C}_{0}$ & & & & & & & & & \\
\hline $\mathrm{T}_{2}$ & $\mathrm{~A}_{0} \mathrm{~B}_{0} \mathrm{C}_{1}$ & $55.85 \mathrm{cde}$ & $56.37 \mathrm{cde}$ & $56.11 \mathrm{def}$ & $61.68 \mathrm{ef}$ & $66.33 \mathrm{bcd}$ & \begin{tabular}{|l|}
$64.01 \mathrm{ef}$ \\
\end{tabular} & 68.93def & 71.30def & $70.12 \mathrm{ef}$ \\
\hline $\mathrm{T}_{3}$ & $\mathrm{~A}_{0} \mathrm{~B}_{1} \mathrm{C}_{0}$ & $49.36 \mathrm{ef}$ & $52.67 \mathrm{de}$ & $51.02 \mathrm{ef}$ & 59.64ef & $61.1 \mathrm{~d}$ & $60.37 \mathrm{ef}$ & $67.15 \mathrm{ef}$ & 67.90ef & $67.53 \mathrm{ef}$ \\
\hline $\mathrm{T}_{4}$ & $\mathrm{~A}_{0} \mathrm{~B}_{1} \mathrm{C}_{1}$ & $56.23 \mathrm{cde}$ & 57.8bcde & $57.02 \mathrm{cde}$ & 62.91def & $66.87 \mathrm{bcd}$ & \begin{tabular}{|l} 
64.89def \\
\end{tabular} & 72.70cdef & 72.80cdef & $72.75 \mathrm{def}$ \\
\hline $\mathrm{T}_{5}$ & $\mathrm{~A}_{0} \mathrm{~B}_{2} \mathrm{C}_{0}$ & $52.65 \mathrm{def}$ & $54.47 \mathrm{de}$ & 53.56def & 61.01ef & $64.27 \mathrm{~cd}$ & 62.64ef & 70.66def & $70.30 \mathrm{ef}$ & $70.48 \mathrm{ef}$ \\
\hline $\mathrm{T}_{6}$ & $\mathrm{~A}_{0} \mathrm{~B}_{2} \mathrm{C}_{1}$ & $57.35 \mathrm{bcde}$ & 59.17bcde & 58.26bce & 63.13def & $68.13 \mathrm{abc}$ & 65.63cdef & $70.23 \mathrm{def}$ & $73.33 \mathrm{cdef}$ & 71.78ef \\
\hline $\mathrm{T}_{7}$ & $\mathrm{~A}_{1} \mathrm{~B}_{0} \mathrm{C}_{0}$ & 64.28abcd & $59.7 \mathrm{bcd}$ & 61.99abcde & $68.47 \mathrm{cde}$ & 68.5abcd & 68.49bcdef & $73.65 \mathrm{cdef}$ & 74.33cdef & $73.99 \mathrm{cde}$ \\
\hline $\mathrm{T}_{8}$ & $\mathrm{~A}_{1} \mathrm{~B}_{0} \mathrm{C}_{1}$ & 62.32abcd & 61.07abcd & 61.70abcde & $69.20 \mathrm{bcde}$ & 69.67abcd & 69.44bcde & $74.87 \mathrm{cde}$ & $76.60 \mathrm{bcde}$ & $75.74 \mathrm{cde}$ \\
\hline $\mathrm{T}_{9}$ & $\mathrm{~A}_{1} \mathrm{~B}_{1} \mathrm{C}_{0}$ & 62.23abcd & 62.33abcd & 62.28abcde & 75.27abc & 70.67abcd & 72.97abcde & $78.60 \mathrm{bcd}$ & $77.73 \mathrm{bcde}$ & $78.17 \mathrm{bcde}$ \\
\hline $\mathrm{T}_{10}$ & $\mathrm{~A}_{1} \mathrm{~B}_{1} \mathrm{C}_{1}$ & $67.93 \mathrm{adc}$ & $70.73 \mathrm{ab}$ & $69.33 \mathrm{abc}$ & $80.62 \mathrm{ab}$ & $79.3 \mathrm{ab}$ & 79.96ab & $86.96 \mathrm{ab}$ & $88.10 \mathrm{ab}$ & $87.53 \mathrm{ab}$ \\
\hline $\mathrm{T}_{11}$ & $\mathrm{~A}_{1} \mathrm{~B}_{2} \mathrm{C}_{0}$ & 69.00ad & $71.8 \mathrm{ab}$ & $70.40 \mathrm{ab}$ & 78.00abc & 77.67abc & 77.84abcd & $82.60 \mathrm{abc}$ & 83.03abcd & 82.82abcd \\
\hline $\mathrm{T}_{12}$ & $\mathrm{~A}_{1} \mathrm{~B}_{2} \mathrm{C}_{1}$ & $72.47 \mathrm{a}$ & $73.93 a$ & $73.20 \mathrm{a}$ & $85.05 a$ & $82.37 \mathrm{a}$ & $83.71 \mathrm{a}$ & $89.98 \mathrm{a}$ & $91.53 \mathrm{a}$ & $90.76 \mathrm{a}$ \\
\hline $\mathrm{T}_{13}$ & $\mathrm{~A}_{2} \mathrm{~B}_{0} \mathrm{C}_{0}$ & 64.95abcd & $59.53 \mathrm{bcde}$ & 62.24abcde & $68.28 \mathrm{cde}$ & 68.4abcd & 68.34bcdef & $73.56 \mathrm{cdef}$ & 73.97cdef & 73.77cdef \\
\hline $\mathrm{T}_{14}$ & $\mathrm{~A}_{2} \mathrm{~B}_{0} \mathrm{C}_{1}$ & 62.91 abcd & 61.73abcd & 62.32abcde & 74.10abcd & 70.33abcd & 72.22abcde & 76.90bcde & 76.90bcde & 76.90bcde \\
\hline $\mathrm{T}_{15}$ & $\mathrm{~A}_{2} \mathrm{~B}_{1} \mathrm{C}_{0}$ & $65.48 \mathrm{abc}$ & 64.2abcd & 64.84abcd & 69.03bcde & 69.63abcd & 69.33bcde & $75.20 \mathrm{cde}$ & 75.50cde & $75.35 \mathrm{cde}$ \\
\hline $\mathrm{T}_{16}$ & $\mathrm{~A}_{2} \mathrm{~B}_{1} \mathrm{C}_{1}$ & $69.85 \mathrm{ad}$ & $68.7 \mathrm{abc}$ & 69.28abc & 78.07abc & 77.4abcd & 77.74abcd & $82.87 \mathrm{abc}$ & $84.10 a b c$ & 83.49abcd \\
\hline $\mathrm{T}_{17}$ & $\mathrm{~A}_{2} \mathrm{~B}_{2} \mathrm{C}_{0}$ & 61.34abcde & $59.77 \mathrm{bcd}$ & 60.56bcde & 69.24bcde & 68.57abcd & 68.91bcdef & $75.20 \mathrm{cde}$ & $75.20 \mathrm{cde}$ & $75.20 \mathrm{cde}$ \\
\hline $\mathrm{T}_{18}$ & $\mathrm{~A}_{2} \mathrm{~B}_{2} \mathrm{C}_{1}$ & $70.31 \mathrm{a}$ & $68.77 \mathrm{abc}$ & $69.54 a b$ & 78.62abc & 77.93abc & \begin{tabular}{|l|}
$78.28 \mathrm{abc}$ \\
\end{tabular} & $83.35 \mathrm{adc}$ & $84.67 \mathrm{abc}$ & $84.01 \mathrm{abc}$ \\
\hline \multicolumn{2}{|c|}{ S.Em. $( \pm)$} & 4.36 & 4.93 & 4.34 & 4.13 & 5.12 & \begin{tabular}{|l|}
4.53 \\
\end{tabular} & 3.87 & 4.38 & 4.53 \\
\hline \multicolumn{2}{|c|}{ L.S.D. $(\mathrm{P} \leq 0.05)$} & 12.54 & 14.16 & 12.47 & 11.86 & 14.70 & 13.03 & 11.13 & 12.58 & 13.03 \\
\hline
\end{tabular}


Table.2 Effect of Nutrient management on Canopy Spread (cm) on North- South Direction

\begin{tabular}{|c|c|c|c|c|c|c|c|c|c|}
\hline \multirow[t]{2}{*}{ Treatments } & \multicolumn{3}{|c|}{3 MAP } & \multicolumn{3}{|c|}{6 MAP } & \multicolumn{3}{|c|}{9 МАР } \\
\hline & 2015 & 2016 & Mean & 2015 & 2016 & Mean & 2015 & 2016 & Mean \\
\hline$\overline{\mathrm{A}_{0}}$ & $21.84 \mathrm{~b}$ & $22.09 \mathrm{~b}$ & $21.97 \mathrm{~b}$ & $30.74 c$ & $33.29 \mathrm{~b}$ & $32.02 \mathrm{c}$ & $44.16 \mathrm{~b}$ & $44.70 \mathrm{c}$ & $44.43 c$ \\
\hline $\mathrm{A}_{1}$ & $25.88 \mathrm{a}$ & $26.48 \mathrm{a}$ & $26.18 \mathrm{a}$ & $40.90 \mathrm{a}$ & $44.42 \mathrm{a}$ & $42.66 \mathrm{a}$ & $55.75 a$ & $57.58 \mathrm{a}$ & $56.67 \mathrm{a}$ \\
\hline $\mathrm{A}_{2}$ & $25.34 \mathrm{a}$ & $25.84 \mathrm{a}$ & $25.59 \mathrm{a}$ & $40.02 \mathrm{~b}$ & $43.16 \mathrm{a}$ & $41.59 \mathrm{~b}$ & $55.23 \mathrm{a}$ & $55.27 \mathrm{~b}$ & $55.25 \mathrm{~b}$ \\
\hline S.Em. ( \pm$)$ & 0.39 & 0.52 & 0.44 & 0.27 & 0.45 & 0.25 & 0.44 & 0.44 & $\mathbf{0 . 4 3}$ \\
\hline L.S.D(P $\leq 0.05)$ & 1.11 & 1.50 & 1.27 & 0.76 & 1.29 & 0.73 & 1.25 & 1.25 & 1.22 \\
\hline $\mathrm{B}_{0}$ & $23.41 \mathrm{~b}$ & $24.03 \mathrm{~b}$ & $23.72 \mathrm{~b}$ & $35.31 \mathrm{~b}$ & $38.31 \mathrm{~b}$ & $36.81 \mathrm{~b}$ & $49.81 \mathrm{~b}$ & $50.26 \mathrm{~b}$ & $50.04 \mathrm{~b}$ \\
\hline $\mathrm{B}_{1}$ & $24.66 \mathrm{a}$ & $24.84 \mathrm{~b}$ & $24.75 \mathrm{ab}$ & $37.80 \mathrm{a}$ & $40.99 a$ & $39.40 \mathrm{a}$ & $52.52 \mathrm{a}$ & $53.32 \mathrm{a}$ & $52.92 \mathrm{a}$ \\
\hline $\mathrm{B}_{2}$ & $25.00 \mathrm{a}$ & $25.54 \mathrm{a}$ & $25.27 \mathrm{a}$ & $38.55 \mathrm{a}$ & $41.57 \mathrm{a}$ & $40.06 a$ & $52.81 \mathrm{a}$ & $53.97 \mathrm{a}$ & $53.39 a$ \\
\hline S.Em. $( \pm)$ & 0.39 & 0.52 & 0.44 & 0.27 & 0.45 & 0.25 & 0.44 & 0.44 & 0.43 \\
\hline L.S.D(P $\leq 0.05)$ & 1.11 & 1.50 & 1.27 & 0.76 & 1.29 & 0.73 & 1.25 & 1.25 & 1.22 \\
\hline $\mathrm{C}_{0}$ & $23.86 \mathrm{~b}$ & $24.19 \mathrm{a}$ & $24.03 \mathrm{~b}$ & $35.51 \mathrm{~b}$ & $39.03 b$ & $37.28 \mathrm{~b}$ & $49.66 \mathrm{~b}$ & $50.56 \mathrm{~b}$ & $50.11 \mathrm{~b}$ \\
\hline $\mathrm{C}_{1}$ & $24.85 \mathrm{a}$ & $25.41 \mathrm{a}$ & $25.13 \mathrm{a}$ & $38.93 a$ & $41.55 \mathrm{a}$ & $40.24 a$ & $53.77 \mathrm{a}$ & $54.47 \mathrm{a}$ & $54.12 \mathrm{a}$ \\
\hline S.Em. $( \pm)$ & $\mathbf{0 . 3 2}$ & 0.43 & 0.36 & 0.22 & $\mathbf{0 . 3 7}$ & 0.21 & 0.36 & 0.36 & $\mathbf{0 . 3 5}$ \\
\hline L.S.D(P $\leq 0.05)$ & 0.91 & NS & 1.03 & 0.62 & 1.05 & 0.59 & 1.02 & 1.02 & 1.00 \\
\hline
\end{tabular}

\begin{tabular}{|c|c|c|c|c|c|c|c|c|c|c|}
\hline \multirow{2}{*}{\multicolumn{2}{|c|}{$\begin{array}{l}\text { Treatments/ } \\
\text { Combination }\end{array}$}} & \multicolumn{3}{|c|}{3 MAP } & \multicolumn{3}{|c|}{6 MAP } & \multicolumn{3}{|c|}{9 MAP } \\
\hline & & 2015 & 2016 & Mean & 2015 & 2016 & Mean & 2015 & 2016 & Mean \\
\hline $\mathrm{T}_{1}$ & $\mathrm{~A}_{0} \mathrm{~B}_{0} \mathrm{C}_{0}$ & $19.84 \mathrm{~g}$ & $19.67 \mathrm{e}$ & $19.76 \mathrm{~g}$ & $27.39 \mathrm{~g}$ & $30.37 \mathrm{~h}$ & $28.88 \mathrm{i}$ & $41.48 \mathrm{~g}$ & $40.59 \mathrm{i}$ & $41.04 \mathrm{i}$ \\
\hline $\mathrm{T}_{2}$ & $\mathrm{~A}_{0} \mathrm{~B}_{0} \mathrm{C}_{1}$ & $23.29 \mathrm{cdef}$ & 23.07bcde & $23.18 \mathrm{cdef}$ & $32.19 \mathrm{e}$ & $33.73 \mathrm{~g}$ & $32.96 \mathrm{fg}$ & $47.08 \mathrm{f}$ & $45.83 \mathrm{gh}$ & $46.46 \mathrm{~g}$ \\
\hline $\mathrm{T}_{3}$ & $\mathrm{~A}_{0} \mathrm{~B}_{1} \mathrm{C}_{0}$ & $21.24 \mathrm{fg}$ & $21.63 \mathrm{de}$ & $21.44 \mathrm{fg}$ & $28.37 \mathrm{fg}$ & $32.82 \mathrm{gh}$ & 30.60hi & $42.47 \mathrm{~g}$ & 43.60hi & 43.04hi \\
\hline $\mathrm{T}_{4}$ & $\mathrm{~A}_{0} \mathrm{~B}_{1} \mathrm{C}_{1}$ & $22.09 \mathrm{efg}$ & $22.24 \mathrm{cde}$ & 22.17 efg & $32.87 \mathrm{e}$ & $34.24 \mathrm{~g}$ & $33.56 \mathrm{fg}$ & $46.70 \mathrm{f}$ & $46.34 \mathrm{gh}$ & $46.52 \mathrm{~g}$ \\
\hline $\mathrm{T}_{5}$ & $\mathrm{~A}_{0} \mathrm{~B}_{2} \mathrm{C}_{0}$ & $22.18 \mathrm{efg}$ & 22.96bcde & $22.57 \mathrm{defg}$ & $29.98 \mathrm{f}$ & $33.62 \mathrm{~g}$ & $31.80 \mathrm{gh}$ & $42.99 \mathrm{~g}$ & $44.75 \mathrm{gh}$ & $43.87 \mathrm{ghi}$ \\
\hline $\mathrm{T}_{6}$ & $\mathrm{~A}_{0} \mathrm{~B}_{2} \mathrm{C}_{1}$ & 22.41defg & 22.96bcde & 22.69defg & $33.66 \mathrm{e}$ & $34.96 \mathrm{~g}$ & $34.31 \mathrm{f}$ & $44.25 \mathrm{fg}$ & $47.06 \mathrm{~g}$ & $45.66 \mathrm{gh}$ \\
\hline $\mathrm{T}_{7}$ & $\mathrm{~A}_{1} \mathrm{~B}_{0} \mathrm{C}_{0}$ & 24.72abcde & 25.72abc & 25.22abcde & $37.39 \mathrm{~cd}$ & $40.13 \mathrm{ef}$ & $38.76 \mathrm{de}$ & $51.98 \mathrm{de}$ & $53.23 \mathrm{ef}$ & $52.6 \mathrm{ef}$ \\
\hline $\mathrm{T}_{8}$ & $\mathrm{~A}_{1} \mathrm{~B}_{0} \mathrm{C}_{1}$ & $24.55 \mathrm{bcde}$ & $25.33 \mathrm{abc}$ & 24.94abcde & $38.73 b c$ & 42.88bcde & $40.81 b c$ & $53.14 \mathrm{cde}$ & $54.98 \mathrm{cde}$ & $54.06 \mathrm{cde}$ \\
\hline $\mathrm{T}_{9}$ & $\mathrm{~A}_{1} \mathrm{~B}_{1} \mathrm{C}_{0}$ & $26.53 \mathrm{ab}$ & $25.98 \mathrm{ab}$ & $26.26 \mathrm{abc}$ & $39.96 \mathrm{~b}$ & 44.64abcd & $42.30 \mathrm{~b}$ & $54.77 \mathrm{~cd}$ & $56.74 \mathrm{~cd}$ & $55.76 \mathrm{bcd}$ \\
\hline $\mathrm{T}_{10}$ & $\mathrm{~A}_{1} \mathrm{~B}_{1} \mathrm{C}_{1}$ & $26.45 \mathrm{ab}$ & $27.24 \mathrm{a}$ & $26.85 \mathrm{ab}$ & $43.12 \mathrm{a}$ & $46.54 \mathrm{a}$ & $44.83 \mathrm{a}$ & $59.35 \mathrm{a}$ & $61.32 \mathrm{a}$ & $60.34 \mathrm{a}$ \\
\hline $\mathrm{T}_{11}$ & $\mathrm{~A}_{1} \mathrm{~B}_{2} \mathrm{C}_{0}$ & $25.68 \mathrm{abc}$ & $27.02 \mathrm{a}$ & $26.35 \mathrm{ab}$ & $42.55 \mathrm{a}$ & 45.68abc & $44.12 \mathrm{a}$ & $55.81 \mathrm{bc}$ & $57.78 \mathrm{bc}$ & $56.80 \mathrm{bc}$ \\
\hline $\mathrm{T}_{12}$ & $\mathrm{~A}_{1} \mathrm{~B}_{2} \mathrm{C}_{1}$ & $27.35 \mathrm{a}$ & $27.60 \mathrm{a}$ & $27.48 \mathrm{a}$ & $43.64 \mathrm{a}$ & $46.64 a$ & $45.14 \mathrm{a}$ & $59.44 \mathrm{a}$ & $61.41 \mathrm{a}$ & $60.43 a$ \\
\hline $\mathrm{T}_{13}$ & $\mathrm{~A}_{2} \mathrm{~B}_{0} \mathrm{C}_{0}$ & $23.68 \mathrm{cdef}$ & 24.68abcd & $24.18 \mathrm{bcdef}$ & $36.32 \mathrm{~d}$ & $39.35 \mathrm{f}$ & $37.84 \mathrm{e}$ & $50.65 \mathrm{e}$ & $51.45 \mathrm{f}$ & $51.05 \mathrm{f}$ \\
\hline $\mathrm{T}_{14}$ & $\mathrm{~A}_{2} \mathrm{~B}_{0} \mathrm{C}_{1}$ & 24.35bcde & $25.71 \mathrm{abc}$ & 25.03abcde & $39.82 \mathrm{~b}$ & $43.38 \mathrm{bcd}$ & $41.60 \mathrm{bc}$ & $54.55 \mathrm{~cd}$ & $55.48 \mathrm{cde}$ & $55.02 \mathrm{cde}$ \\
\hline $\mathrm{T}_{15}$ & $\mathrm{~A}_{2} \mathrm{~B}_{1} \mathrm{C}_{0}$ & $25.08 \mathrm{abcd}$ & $24.8 \mathrm{abcd}$ & 24.94abcde & $39.34 \mathrm{~b}$ & $42.05 \mathrm{def}$ & $40.70 \mathrm{bc}$ & $53.25 \mathrm{cde}$ & $54.15 \mathrm{def}$ & 53.70def \\
\hline $\mathrm{T}_{16}$ & $\mathrm{~A}_{2} \mathrm{~B}_{1} \mathrm{C}_{1}$ & $26.55 \mathrm{ab}$ & $27.15 \mathrm{a}$ & $26.85 \mathrm{ab}$ & $43.16 \mathrm{a}$ & 45.67abc & $44.42 \mathrm{a}$ & $58.55 \mathrm{ab}$ & $57.75 b c$ & $58.15 \mathrm{ab}$ \\
\hline $\mathrm{T}_{17}$ & $\mathrm{~A}_{2} \mathrm{~B}_{2} \mathrm{C}_{0}$ & $25.81 \mathrm{abc}$ & 25.31 abcd & $25.56 \mathrm{abcd}$ & $38.32 \mathrm{bc}$ & $42.64 \mathrm{cde}$ & $40.48 \mathrm{~cd}$ & $53.52 \mathrm{cde}$ & 52.74ef & 53.13def \\
\hline $\mathrm{T}_{18}$ & $\mathrm{~A}_{2} \mathrm{~B}_{2} \mathrm{C}_{1}$ & $26.56 \mathrm{ab}$ & $27.39 \mathrm{a}$ & $26.98 \mathrm{ab}$ & $43.15 \mathrm{a}$ & $45.88 \mathrm{ab}$ & $44.52 \mathrm{a}$ & $60.85 \mathrm{a}$ & $60.05 \mathrm{ab}$ & $60.45 a$ \\
\hline \multicolumn{2}{|c|}{ S.Em. $( \pm)$} & 0.65 & 1.28 & 1.08 & 0.65 & 1.10 & 0.62 & 1.07 & 1.07 & 1.04 \\
\hline \multicolumn{2}{|c|}{ L.S.D. $(\mathrm{P}<0.05)$} & 2.72 & 3.67 & 3.10 & 1.86 & 3.16 & 1.78 & 3.06 & 3.07 & 2.99 \\
\hline
\end{tabular}

MAP-Month after planting

**Means with the same letter are not significantly different 
Table.2 Effect of Nutrient management on Canopy Spread (cm) on North- South Direction(contd....)

\begin{tabular}{|c|c|c|c|c|c|c|c|c|c|}
\hline \multirow[t]{2}{*}{ Treatments } & \multicolumn{3}{|c|}{12 MAP } & \multicolumn{3}{|c|}{15 MAP } & \multicolumn{3}{|c|}{18 MAP } \\
\hline & 2015 & 2016 & Mean & 2015 & 2016 & Mean & 2015 & 2016 & Mean \\
\hline$\overline{\mathrm{A}_{0}}$ & $58.64 \mathrm{c}$ & $59.11 \mathrm{c}$ & $58.88 \mathrm{c}$ & $73.10 \mathrm{~b}$ & $76.06 \mathrm{~b}$ & $74.58 b$ & $81.86 \mathrm{~b}$ & $84.13 b$ & $83.00 \mathrm{~b}$ \\
\hline $\mathrm{A}_{1}$ & $75.52 \mathrm{a}$ & $74.91 \mathrm{a}$ & $73.22 \mathrm{a}$ & $89.32 \mathrm{a}$ & $92.02 \mathrm{a}$ & $90.67 \mathrm{a}$ & $107.54 \mathrm{a}$ & $109.18 \mathrm{a}$ & $108.36 \mathrm{a}$ \\
\hline $\mathrm{A}_{2}$ & $72.71 \mathrm{~b}$ & $73.08 \mathrm{~b}$ & $72.90 \mathrm{~b}$ & $85.59 \mathrm{a}$ & $88.26 \mathrm{a}$ & $86.93 a$ & $104.96 \mathrm{a}$ & $106.78 \mathrm{a}$ & $105.87 \mathrm{a}$ \\
\hline S.Em. ( \pm$)$ & 0.49 & 0.43 & 0.45 & 2.36 & 2.43 & 2.39 & 1.25 & 2.20 & 1.62 \\
\hline L.S.D(P $\leq 0.05)$ & 1.42 & 1.22 & 1.29 & 6.78 & 6.97 & 6.87 & 3.59 & 6.31 & 4.65 \\
\hline $\mathrm{B}_{0}$ & $66.05 \mathrm{~b}$ & $66.07 \mathrm{~b}$ & $66.06 \mathrm{~b}$ & $78.09 \mathrm{~b}$ & $81.18 \mathrm{~b}$ & $79.64 b$ & $93.57 \mathrm{~b}$ & $94.79 \mathrm{~b}$ & $94.18 \mathrm{~b}$ \\
\hline $\mathrm{B}_{1}$ & $70.05 a$ & $70.23 a$ & $70.14 a$ & $83.89 \mathrm{~b}$ & $86.51 \mathrm{ab}$ & $85.20 \mathrm{ab}$ & $99.57 \mathrm{a}$ & $101.49 \mathrm{a}$ & $100.53 \mathrm{a}$ \\
\hline $\mathrm{B}_{2}$ & $70.77 \mathrm{a}$ & $70.81 \mathrm{a}$ & $70.79 a$ & $86.03 a$ & $88.65 \mathrm{a}$ & $87.34 \mathrm{a}$ & $101.24 \mathrm{a}$ & $103.81 \mathrm{a}$ & $102.52 \mathrm{a}$ \\
\hline S.Em. $( \pm)$ & 0.49 & 0.43 & 0.45 & 2.36 & 2.43 & 2.39 & 1.25 & 2.20 & 1.62 \\
\hline L.S.D(P $\leq \mathbf{0 . 0 5})$ & 1.42 & 1.22 & 1.29 & 6.78 & 6.97 & 6.87 & 3.59 & 6.31 & 4.65 \\
\hline $\mathrm{C}_{0}$ & $65.82 \mathrm{~b}$ & $65.57 \mathrm{~b}$ & $65.70 \mathrm{~b}$ & $78.88 \mathrm{~b}$ & $81.69 \mathrm{~b}$ & $80.29 \mathrm{~b}$ & $92.99 \mathrm{~b}$ & $94.35 b$ & $93.67 \mathrm{~b}$ \\
\hline $\mathrm{C}_{1}$ & $72.10 \mathrm{a}$ & $72.50 \mathrm{a}$ & $72.30 \mathrm{a}$ & $86.46 \mathrm{a}$ & $89.20 \mathrm{a}$ & $87.83 a$ & $103.26 \mathrm{a}$ & $105.71 \mathrm{a}$ & $104.49 \mathrm{a}$ \\
\hline S.Em. $( \pm)$ & 0.40 & 0.34 & 0.37 & 1.92 & 1.98 & 1.95 & 1.02 & 1.79 & 1.32 \\
\hline L.S.D(P $\leq 0.05)$ & 1.16 & 1.00 & 1.05 & 5.53 & 5.69 & 5.61 & 2.93 & 5.15 & 3.80 \\
\hline
\end{tabular}

\begin{tabular}{|c|c|c|c|c|c|c|c|c|c|c|}
\hline \multirow{2}{*}{\multicolumn{2}{|c|}{$\begin{array}{l}\text { Treatments/ } \\
\text { Combination }\end{array}$}} & \multicolumn{3}{|c|}{12 MAP } & \multicolumn{3}{|c|}{15 MAP } & \multicolumn{3}{|c|}{18 MAP } \\
\hline & & 2015 & 2016 & Mean & 2015 & 2016 & Mean & 2015 & 2016 & Mean \\
\hline $\mathrm{T}_{1}$ & $\mathrm{~A}_{0} \mathrm{~B}_{0} \mathrm{C}_{0}$ & $53.06 \mathrm{i}$ & $53.59 \mathrm{~g}$ & $53.33 \mathrm{i}$ & $61.23 \mathrm{e}$ & $65.1 \mathrm{e}$ & $63.17 \mathrm{e}$ & $67.74 \mathrm{~g}$ & $68.6 \mathrm{f}$ & $68.17 \mathrm{i}$ \\
\hline $\mathrm{T}_{2}$ & $\mathrm{~A}_{0} \mathrm{~B}_{0} \mathrm{C}_{1}$ & $61.08 \mathrm{~g}$ & $61.53 \mathrm{e}$ & $61.31 \mathrm{~g}$ & $75.32 \mathrm{cdc}$ & $78.33 \mathrm{cde}$ & $76.83 \mathrm{cde}$ & $86.16 \mathrm{de}$ & $86.9 \mathrm{cde}$ & $86.53 \mathrm{gh}$ \\
\hline $\mathrm{T}_{3}$ & $\mathrm{~A}_{0} \mathrm{~B}_{1} \mathrm{C}_{0}$ & 56.01hi & $56.60 \mathrm{f}$ & 56.31hi & $70.56 \mathrm{de}$ & $73.17 \mathrm{de}$ & $71.87 \mathrm{de}$ & $76.30 \mathrm{fg}$ & $77.83 \mathrm{ef}$ & $77.07 \mathrm{hi}$ \\
\hline $\mathrm{T}_{4}$ & $\mathrm{~A}_{0} \mathrm{~B}_{1} \mathrm{C}_{1}$ & $61.27 \mathrm{~g}$ & $62.04 \mathrm{e}$ & $61.66 \mathrm{~g}$ & $78.04 \mathrm{bcd}$ & 80.63 bcde & $79.34 \mathrm{bcde}$ & $90.71 \mathrm{~d}$ & $94.73 \mathrm{bcd}$ & $92.72 \mathrm{fg}$ \\
\hline $\mathrm{T}_{5}$ & $\mathrm{~A}_{0} \mathrm{~B}_{2} \mathrm{C}_{0}$ & $57.23 \mathrm{~h}$ & $57.75 \mathrm{f}$ & $57.49 \mathrm{~h}$ & $74.87 \mathrm{cde}$ & $77.43 \mathrm{cde}$ & $76.15 \mathrm{cde}$ & $78.87 \mathrm{ef}$ & 79.77def & 79.32hi \\
\hline $\mathrm{T}_{6}$ & $\mathrm{~A}_{0} \mathrm{~B}_{2} \mathrm{C}_{1}$ & $63.19 \mathrm{~g}$ & $63.16 \mathrm{e}$ & $63.18 \mathrm{~g}$ & $78.57 \mathrm{bcd}$ & $81.67 \mathrm{bcde}$ & $80.12 \mathrm{bcd}$ & $91.41 \mathrm{~cd}$ & $96.93 b c$ & 94.17efg \\
\hline $\mathrm{T}_{7}$ & $\mathrm{~A}_{1} \mathrm{~B}_{0} \mathrm{C}_{0}$ & $69.73 \mathrm{ef}$ & $68.93 \mathrm{~cd}$ & $69.33 \mathrm{ef}$ & $81.47 \mathrm{bcd}$ & $84.2 \mathrm{bcde}$ & $82.84 \mathrm{bcd}$ & $100.40 \mathrm{~b}$ & $101.07 \mathrm{bc}$ & $100.74 \mathrm{def}$ \\
\hline $\mathrm{T}_{8}$ & $\mathrm{~A}_{1} \mathrm{~B}_{0} \mathrm{C}_{1}$ & $71.98 \mathrm{de}$ & $71.31 \mathrm{bc}$ & $71.65 \mathrm{cde}$ & $84.07 \mathrm{bcd}$ & $87.57 \mathrm{abcd}$ & $85.82 \mathrm{bcd}$ & $103.74 b$ & $104.93 \mathrm{ab}$ & 104.34de \\
\hline $\mathrm{T}_{9}$ & $\mathrm{~A}_{1} \mathrm{~B}_{1} \mathrm{C}_{0}$ & $74.40 \mathrm{~cd}$ & $73.44 b$ & $73.92 \mathrm{c}$ & 86.47abcd & 89.23abcd & 87.85abcd & $105.07 \mathrm{~b}$ & $106.4 \mathrm{ab}$ & $105.74 \mathrm{bcd}$ \\
\hline $\mathrm{T}_{10}$ & $\mathrm{~A}_{1} \mathrm{~B}_{1} \mathrm{C}_{1}$ & $80.39 \mathrm{ab}$ & $80.27 \mathrm{a}$ & $80.33 \mathrm{ab}$ & $94.31 \mathrm{ab}$ & $96.97 \mathrm{ab}$ & $95.64 \mathrm{ab}$ & $116.17 \mathrm{a}$ & $116.83 a$ & $116.50 \mathrm{ab}$ \\
\hline $\mathrm{T}_{11}$ & $\mathrm{~A}_{1} \mathrm{~B}_{2} \mathrm{C}_{0}$ & $74.36 \mathrm{~d}$ & $73.89 \mathrm{~b}$ & $74.13 \mathrm{c}$ & 87.13abcd & 89.6abcd & 88.37abcd & $103.57 \mathrm{~b}$ & $106.9 \mathrm{ab}$ & $105.24 \mathrm{bcde}$ \\
\hline $\mathrm{T}_{12}$ & $\mathrm{~A}_{1} \mathrm{~B}_{2} \mathrm{C}_{1}$ & $82.28 \mathrm{a}$ & $81.61 \mathrm{a}$ & $81.95 \mathrm{a}$ & $102.48 \mathrm{a}$ & $104.57 \mathrm{a}$ & $103.53 \mathrm{a}$ & $116.30 \mathrm{a}$ & $118.97 \mathrm{a}$ & $117.64 \mathrm{a}$ \\
\hline $\mathrm{T}_{13}$ & $\mathrm{~A}_{2} \mathrm{~B}_{0} \mathrm{C}_{0}$ & $68.08 \mathrm{f}$ & $67.65 \mathrm{~d}$ & $67.87 \mathrm{f}$ & $80.34 \mathrm{bcd}$ & $83.07 \mathrm{bcde}$ & $81.71 \mathrm{bcd}$ & $99.67 \mathrm{bc}$ & $101 b c$ & 100.34def \\
\hline $\mathrm{T}_{14}$ & $\mathrm{~A}_{2} \mathrm{~B}_{0} \mathrm{C}_{1}$ & 72.36de & $73.38 \mathrm{~b}$ & $72.87 \mathrm{~cd}$ & 86.13abcd & $88.8 \mathrm{abcd}$ & 87.47abcd & $103.68 \mathrm{~b}$ & $106.27 \mathrm{ab}$ & $104.98 \mathrm{cde}$ \\
\hline $\mathrm{T}_{15}$ & $\mathrm{~A}_{2} \mathrm{~B}_{1} \mathrm{C}_{0}$ & $70.37 \mathrm{ef}$ & $69.85 \mathrm{~cd}$ & 70.11def & $84.20 \mathrm{bcd}$ & $86.93 \mathrm{bcde}$ & $85.57 \mathrm{bcd}$ & $103.40 \mathrm{~b}$ & $104.07 \mathrm{ab}$ & 103.74def \\
\hline $\mathrm{T}_{16}$ & $\mathrm{~A}_{2} \mathrm{~B}_{1} \mathrm{C}_{1}$ & $77.86 \mathrm{bc}$ & $79.19 \mathrm{a}$ & $78.53 b$ & $89.75 a b c$ & $92.1 \mathrm{abc}$ & $90.93 \mathrm{abc}$ & $105.77 \mathrm{~b}$ & $109.1 \mathrm{ab}$ & 107.44abcd \\
\hline $\mathrm{T}_{17}$ & $\mathrm{~A}_{2} \mathrm{~B}_{2} \mathrm{C}_{0}$ & 69.11ef & $68.44 \mathrm{~cd}$ & $68.78 \mathrm{ef}$ & $83.65 \mathrm{bcd}$ & $86.47 \mathrm{bcde}$ & $85.06 \mathrm{bcd}$ & $101.87 \mathrm{~b}$ & $103.53 \mathrm{ab}$ & 102.70def \\
\hline $\mathrm{T}_{18}$ & $\mathrm{~A}_{2} \mathrm{~B}_{2} \mathrm{C}_{1}$ & $78.47 \mathrm{~b}$ & $79.98 \mathrm{a}$ & $79.23 \mathrm{ab}$ & $89.46 \mathrm{abc}$ & $92.17 \mathrm{abc}$ & $90.82 \mathrm{abc}$ & $115.40 \mathrm{a}$ & $116.73 \mathrm{a}$ & $116.07 \mathrm{abc}$ \\
\hline \multicolumn{2}{|c|}{ S.Em. $( \pm)$} & 1.21 & 1.04 & 1.10 & 5.77 & 5.94 & 5.85 & 3.06 & 5.38 & 3.96 \\
\hline \multicolumn{2}{|c|}{ L.S.D. $(\mathrm{P} \leq 0.05)$} & 3.48 & 2.99 & 3.15 & 16.59 & 17.07 & 16.82 & 8.80 & 15.46 & 11.39 \\
\hline
\end{tabular}


Table.3 Effect of Nutrient management on Canopy Spread (cm) on East- West Direction

\begin{tabular}{|c|c|c|c|c|c|c|c|c|c|}
\hline \multirow[t]{2}{*}{ Treatments } & \multicolumn{3}{|c|}{3 MAP } & \multicolumn{3}{|c|}{6 MAP } & \multicolumn{3}{|c|}{9 MAP } \\
\hline & 2015 & 2016 & Mean & 2015 & 2016 & Mean & 2015 & 2016 & Mean \\
\hline$\overline{\mathrm{A}_{0}}$ & $26.80 \mathrm{~b}$ & $27.29 \mathrm{~b}$ & $27.05 b$ & $38.91 b$ & $39.66 \mathrm{~b}$ & $39.28 b$ & $49.12 \mathrm{c}$ & $49.54 c$ & $49.33 c$ \\
\hline $\mathrm{A}_{1}$ & $30.34 \mathrm{a}$ & $31.66 \mathrm{a}$ & $31.28 \mathrm{a}$ & $52.18 \mathrm{a}$ & $52.39 \mathrm{a}$ & $52.29 \mathrm{a}$ & $61.93 a$ & $63.28 \mathrm{a}$ & $62.60 \mathrm{a}$ \\
\hline$A_{2}$ & $30.90 \mathrm{a}$ & $30.97 \mathrm{a}$ & $30.66 \mathrm{a}$ & $50.72 \mathrm{a}$ & $51.00 \mathrm{a}$ & $50.86 \mathrm{a}$ & $59.90 \mathrm{~b}$ & $61.34 \mathrm{~b}$ & $60.62 \mathrm{~b}$ \\
\hline S.Em. ( $( \pm)$ & 0.44 & $\mathbf{0 . 5 3}$ & 0.48 & 0.62 & 0.61 & 0.61 & 0.36 & 0.38 & 0.31 \\
\hline L.S.D(P $\leq 0.05)$ & 1.27 & 1.51 & 1.38 & 1.80 & 1.75 & 1.76 & 1.03 & 1.08 & 0.88 \\
\hline $\mathrm{B}_{0}$ & $28.67 \mathrm{a}$ & $29.22 \mathrm{a}$ & $28.95 \mathrm{a}$ & $45.99 \mathrm{~b}$ & $46.20 \mathrm{~b}$ & $46.10 \mathrm{~b}$ & $55.38 \mathrm{~b}$ & $56.02 \mathrm{c}$ & $55.70 \mathrm{~b}$ \\
\hline $\mathrm{B}_{1}$ & $29.44 \mathrm{a}$ & $29.99 \mathrm{a}$ & $29.72 a$ & $47.54 a b$ & $48.11 \mathrm{a}$ & $47.83 \mathrm{ab}$ & $57.77 \mathrm{a}$ & $58.45 \mathrm{~b}$ & $58.11 \mathrm{a}$ \\
\hline $\mathrm{B}_{2}$ & $29.92 \mathrm{a}$ & $30.70 \mathrm{a}$ & $30.31 \mathrm{a}$ & $48.27 \mathrm{a}$ & $48.74 a$ & $48.51 \mathrm{a}$ & $57.77 \mathrm{a}$ & $59.68 \mathrm{a}$ & $58.74 a$ \\
\hline S.Em. $( \pm)$ & 0.44 & $\mathbf{0 . 5 3}$ & 0.48 & 0.62 & 0.61 & 0.61 & 0.36 & 0.38 & 0.31 \\
\hline L.S.D(P $\leq 0.05)$ & NS & NS & NS & 1.80 & 1.75 & 1.76 & 1.03 & 1.08 & 0.88 \\
\hline $\mathrm{C}_{0}$ & $28.89 \mathrm{a}$ & $29.39 \mathrm{a}$ & $29.14 \mathrm{a}$ & $45.39 \mathrm{~b}$ & $45.69 \mathrm{~b}$ & $45.54 b$ & $54.79 \mathrm{~b}$ & $55.43 \mathrm{~b}$ & $55.11 \mathrm{~b}$ \\
\hline $\mathrm{C}_{1}$ & $29.80 \mathrm{a}$ & $30.55 \mathrm{a}$ & $30.18 \mathrm{a}$ & $49.15 \mathrm{a}$ & $49.68 \mathrm{a}$ & $49.41 \mathrm{a}$ & $59.17 \mathrm{a}$ & $60.67 \mathrm{a}$ & $59.92 \mathrm{a}$ \\
\hline S.Em. $( \pm)$ & 0.36 & 0.43 & 0.39 & 0.51 & 0.50 & 0.50 & 0.29 & 0.31 & 0.25 \\
\hline L.S.D(P $\leq 0.05)$ & NS & NS & NS & 1.47 & 1.43 & 1.43 & 0.84 & 0.88 & 0.72 \\
\hline
\end{tabular}

\begin{tabular}{|c|c|c|c|c|c|c|c|c|c|c|}
\hline \multirow{2}{*}{\multicolumn{2}{|c|}{$\begin{array}{l}\text { Treatments/ } \\
\text { Combination }\end{array}$}} & \multicolumn{3}{|c|}{3 MAP } & \multicolumn{3}{|c|}{6 MAP } & \multicolumn{3}{|c|}{9 MАР } \\
\hline & & 2015 & 2016 & Mean & 2015 & 2016 & Mean & 2015 & 2016 & Mean \\
\hline $\mathrm{T}_{1}$ & $\mathrm{~A}_{0} \mathrm{~B}_{0} \mathrm{C}_{0}$ & $24.46 \mathrm{e}$ & $24.87 \mathrm{e}$ & $24.67 \mathrm{f}$ & $34.25 \mathrm{~g}$ & $35.30 \mathrm{~g}$ & $34.78 \mathrm{f}$ & $46.01 \mathrm{i}$ & $44.76 \mathrm{j}$ & $45.39 \mathrm{i}$ \\
\hline $\mathrm{T}_{2}$ & $\mathrm{~A}_{0} \mathrm{~B}_{0} \mathrm{C}_{1}$ & $28.66 \mathrm{bcd}$ & $28.27 \mathrm{cde}$ & 28.47 bcde & $40.22 \mathrm{f}$ & $41.27 \mathrm{f}$ & $40.75 \mathrm{e}$ & $50.44 \mathrm{~h}$ & $51.26 \mathrm{gh}$ & $50.85 \mathrm{fg}$ \\
\hline $\mathrm{T}_{3}$ & $\mathrm{~A}_{0} \mathrm{~B}_{1} \mathrm{C}_{0}$ & 27.32 cde & $26.83 \mathrm{de}$ & $27.08 \mathrm{def}$ & $38.04 \mathrm{fg}$ & $38.82 \mathrm{fg}$ & $38.43 \mathrm{ef}$ & $48.57 \mathrm{~h}$ & $47.83 \mathrm{i}$ & $48.20 \mathrm{~h}$ \\
\hline $\mathrm{T}_{4}$ & $\mathrm{~A}_{0} \mathrm{~B}_{1} \mathrm{C}_{1}$ & $26.48 \mathrm{de}$ & $27.44 \mathrm{cde}$ & $26.96 \mathrm{ef}$ & $40.22 \mathrm{f}$ & $41.00 \mathrm{f}$ & $40.61 \mathrm{e}$ & $50.64 \mathrm{~h}$ & $51.79 \mathrm{gh}$ & $51.22 \mathrm{f}$ \\
\hline $\mathrm{T}_{5}$ & $\mathrm{~A}_{0} \mathrm{~B}_{2} \mathrm{C}_{0}$ & $27.29 \mathrm{cde}$ & 28.16bcde & $27.73 \mathrm{cdef}$ & $38.59 \mathrm{fg}$ & $39.37 \mathrm{fg}$ & $38.98 \mathrm{ef}$ & $48.81 \mathrm{~h}$ & 49.21hi & 49.01gh \\
\hline $\mathrm{T}_{6}$ & $\mathrm{~A}_{0} \mathrm{~B}_{2} \mathrm{C}_{1}$ & $26.59 \mathrm{de}$ & 28.16bcde & $27.38 \mathrm{cdef}$ & $42.11 \mathrm{f}$ & $42.16 \mathrm{f}$ & $42.14 \mathrm{e}$ & $50.22 \mathrm{~h}$ & $52.37 \mathrm{~g}$ & $51.30 \mathrm{f}$ \\
\hline $\mathrm{T}_{7}$ & $\mathrm{~A}_{1} \mathrm{~B}_{0} \mathrm{C}_{0}$ & $30.05 \mathrm{abc}$ & $30.92 \mathrm{abc}$ & 30.49abc & $49.22 \mathrm{cde}$ & $48.25 \mathrm{de}$ & $48.74 \mathrm{~cd}$ & $57.38 \mathrm{~g}$ & 59.02ef & $58.20 \mathrm{e}$ \\
\hline $\mathrm{T}_{8}$ & $\mathrm{~A}_{1} \mathrm{~B}_{0} \mathrm{C}_{1}$ & $29.73 \mathrm{abc}$ & $30.63 \mathrm{abc}$ & 30.18abcde & 51.36abcde & $52.25 \mathrm{abcd}$ & 51.81abcd & 60.96de & $61.75 \mathrm{~d}$ & $61.36 \mathrm{~d}$ \\
\hline $\mathrm{T}_{9}$ & $\mathrm{~A}_{1} \mathrm{~B}_{1} \mathrm{C}_{0}$ & $30.24 a b c$ & $31.18 \mathrm{ab}$ & $30.71 \mathrm{abc}$ & $50.53 \mathrm{bcde}$ & 51.31bcde & $50.92 \mathrm{bcd}$ & $61.45 \mathrm{cde}$ & $61.32 \mathrm{de}$ & $61.39 \mathrm{~d}$ \\
\hline $\mathrm{T}_{10}$ & $\mathrm{~A}_{1} \mathrm{~B}_{1} \mathrm{C}_{1}$ & $31.25 \mathrm{ab}$ & $32.31 \mathrm{a}$ & $31.78 \mathrm{ab}$ & $54.39 \mathrm{ab}$ & $53.9 \mathrm{ab}$ & $54.15 \mathrm{ab}$ & $65.12 \mathrm{ab}$ & 66.70ab & $65.91 \mathrm{ab}$ \\
\hline $\mathrm{T}_{11}$ & $\mathrm{~A}_{1} \mathrm{~B}_{2} \mathrm{C}_{0}$ & $31.86 \mathrm{a}$ & $32.22 \mathrm{a}$ & $32.04 \mathrm{a}$ & 52.54abcd & $52.8 \mathrm{abc}$ & $52.67 \mathrm{abc}$ & 59.92ef & $62.75 \mathrm{~cd}$ & $61.34 d$ \\
\hline $\mathrm{T}_{12}$ & $\mathrm{~A}_{1} \mathrm{~B}_{2} \mathrm{C}_{1}$ & $32.25 \mathrm{a}$ & $32.68 \mathrm{a}$ & $32.47 \mathrm{a}$ & $55.04 \mathrm{a}$ & $55.84 \mathrm{a}$ & $55.44 \mathrm{a}$ & $66.74 a$ & $68.12 a$ & $67.43 a$ \\
\hline $\mathrm{T}_{13}$ & $\mathrm{~A}_{2} \mathrm{~B}_{0} \mathrm{C}_{0}$ & 29.18abcd & 29.88abcd & 29.53abcde & $48.77 \mathrm{de}$ & $47.88 \mathrm{e}$ & $48.33 \mathrm{~d}$ & $56.14 \mathrm{~g}$ & $57.1 \mathrm{f}$ & $56.62 \mathrm{e}$ \\
\hline $\mathrm{T}_{14}$ & $\mathrm{~A}_{2} \mathrm{~B}_{0} \mathrm{C}_{1}$ & $29.97 \mathrm{abc}$ & $30.78 \mathrm{abc}$ & 30.38abcd & 52.13abcde & $52.25 \mathrm{abcd}$ & 52.19abcd & $61.38 \mathrm{cde}$ & $62.21 \mathrm{~cd}$ & $61.80 \mathrm{~cd}$ \\
\hline $\mathrm{T}_{15}$ & $\mathrm{~A}_{2} \mathrm{~B}_{1} \mathrm{C}_{0}$ & $29.81 \mathrm{abc}$ & $30.17 \mathrm{abcd}$ & 29.91abcde & $48.67 \mathrm{de}$ & $49.45 \mathrm{cde}$ & $49.06 \mathrm{~cd}$ & $57.43 \mathrm{fg}$ & $58.61 \mathrm{f}$ & $58.02 \mathrm{e}$ \\
\hline $\mathrm{T}_{16}$ & $\mathrm{~A}_{2} \mathrm{~B}_{1} \mathrm{C}_{1}$ & $31.57 \mathrm{ab}$ & $32.22 \mathrm{a}$ & $31.90 \mathrm{a}$ & 53.41abc & $54.19 \mathrm{ab}$ & $53.80 \mathrm{ab}$ & $63.42 \mathrm{bcd}$ & $64.44 b c$ & $63.93 b c$ \\
\hline $\mathrm{T}_{17}$ & $\mathrm{~A}_{2} \mathrm{~B}_{2} \mathrm{C}_{0}$ & $29.81 \mathrm{abc}$ & 30.52abcd & 30.17abcde & $47.93 \mathrm{e}$ & 48.04de & $47.99 \mathrm{~d}$ & $57.41 \mathrm{fg}$ & $58.23 \mathrm{f}$ & $57.82 \mathrm{e}$ \\
\hline $\mathrm{T}_{18}$ & $\mathrm{~A}_{2} \mathrm{~B}_{2} \mathrm{C}_{1}$ & $31.71 \mathrm{ab}$ & $32.46 \mathrm{a}$ & $32.09 \mathrm{a}$ & $53.44 \mathrm{abc}$ & $54.22 \mathrm{ab}$ & $53.83 \mathrm{ab}$ & $63.61 b c$ & $67.42 \mathrm{a}$ & $65.52 \mathrm{ab}$ \\
\hline \multicolumn{2}{|c|}{ S.Em. $( \pm)$} & 1.08 & 1.29 & 1.18 & 1.53 & 1.49 & 1.50 & 0.88 & 0.92 & 0.75 \\
\hline \multicolumn{2}{|c|}{ L.S.D. $(\mathrm{P} \leq 0.05)$} & 3.10 & 3.70 & 3.39 & 4.40 & 4.29 & 4.30 & 2.53 & 2.65 & 2.15 \\
\hline
\end{tabular}


Table.3 Effect of Nutrient management on Canopy Spread (cm) on East- West Direction (contd....)

\begin{tabular}{|c|c|c|c|c|c|c|c|c|c|}
\hline \multirow[t]{2}{*}{ Treatments } & \multicolumn{3}{|c|}{12 MAP } & \multicolumn{3}{|c|}{15 MAP } & \multicolumn{3}{|c|}{18 MAP } \\
\hline & 2015 & 2016 & Mean & 2015 & 2016 & Mean & 2015 & 2016 & Mean \\
\hline $\mathrm{A}_{0}$ & $59.71 \mathrm{~b}$ & $61.35 \mathrm{c}$ & $60.53 c$ & $68.76 \mathrm{~b}$ & $74.62 b$ & $71.69 \mathrm{~b}$ & $86.14 \mathrm{~b}$ & $85.87 \mathrm{~b}$ & $86.01 \mathrm{~b}$ \\
\hline $\mathrm{A}_{1}$ & $78.56 \mathrm{a}$ & $77.78 \mathrm{a}$ & $78.17 \mathrm{a}$ & $91.23 \mathrm{a}$ & $92.69 \mathrm{a}$ & $91.96 \mathrm{a}$ & $112.82 \mathrm{a}$ & $112.68 \mathrm{a}$ & $112.75 \mathrm{a}$ \\
\hline $\mathrm{A}_{2}$ & $77.12 \mathrm{a}$ & $75.95 \mathrm{~b}$ & $76.54 \mathrm{~b}$ & $86.85 a$ & $87.87 \mathrm{a}$ & $87.36 \mathrm{a}$ & $107.53 \mathrm{a}$ & $107.82 \mathrm{a}$ & $107.67 \mathrm{a}$ \\
\hline S.Em. ( \pm$)$ & 0.53 & 0.43 & 0.43 & 2.97 & 3.02 & 2.96 & 2.57 & 2.39 & 2.33 \\
\hline L.S.D(P $\leq 0.05)$ & 1.52 & 1.22 & 1.23 & 8.53 & 8.68 & 8.52 & 6.53 & 6.87 & 6.69 \\
\hline $\mathrm{B}_{0}$ & $68.91 \mathrm{~b}$ & $68.73 \mathrm{~b}$ & $68.82 \mathrm{~b}$ & $76.99 \mathrm{~b}$ & $79.87 \mathrm{a}$ & $78.43 \mathrm{~b}$ & $98.27 \mathrm{~b}$ & $98.06 \mathrm{a}$ & $98.17 \mathrm{~b}$ \\
\hline $\mathrm{B}_{1}$ & $72.76 \mathrm{a}$ & $72.89 \mathrm{a}$ & $72.82 \mathrm{a}$ & $83.70 \mathrm{~b}$ & $87.10 \mathrm{a}$ & $85.40 \mathrm{~b}$ & $102.67 \mathrm{~b}$ & $103.57 \mathrm{a}$ & 103.12ab \\
\hline $\mathrm{B}_{2}$ & $73.72 \mathrm{a}$ & $73.47 \mathrm{a}$ & $73.60 \mathrm{a}$ & $86.14 a$ & $88.22 \mathrm{a}$ & $87.18 \mathrm{a}$ & $105.55 \mathrm{a}$ & $104.73 a$ & $105.14 \mathrm{a}$ \\
\hline S.Em. ( \pm$)$ & 0.53 & 0.43 & 0.43 & 2.97 & 3.02 & 2.96 & 2.27 & 2.39 & 2.33 \\
\hline L.S.D(P $\leq 0.05)$ & 1.52 & 1.22 & 1.23 & 8.53 & NS & 8.52 & 6.53 & NS & 6.69 \\
\hline $\mathrm{C}_{0}$ & $68.45 \mathrm{~b}$ & $67.81 \mathrm{~b}$ & $68.13 b$ & $79.13 \mathrm{a}$ & $80.56 \mathrm{~b}$ & $79.85 b$ & $97.48 \mathrm{~b}$ & $97.85 \mathrm{~b}$ & $97.67 \mathrm{~b}$ \\
\hline $\mathrm{C}_{1}$ & $75.14 \mathrm{a}$ & $75.58 \mathrm{a}$ & $75.36 \mathrm{a}$ & $85.42 \mathrm{a}$ & $89.56 \mathrm{a}$ & $87.49 \mathrm{a}$ & $106.85 \mathrm{a}$ & $106.39 a$ & $106.12 \mathrm{a}$ \\
\hline S.Em. ( \pm$)$ & 0.43 & 0.35 & 0.35 & 2.42 & 2.47 & 2.42 & 1.86 & 1.95 & 1.90 \\
\hline L.S.D(P $\leq 0.05)$ & 1.24 & 1.00 & 1.00 & NS & 7.09 & 6.95 & 5.34 & 5.61 & 5.46 \\
\hline
\end{tabular}

\begin{tabular}{|c|c|c|c|c|c|c|c|c|c|c|}
\hline \multirow{2}{*}{\multicolumn{2}{|c|}{$\begin{array}{l}\text { Treatments/ } \\
\text { Combination }\end{array}$}} & \multicolumn{3}{|c|}{12 MAP } & \multicolumn{3}{|c|}{15 MAP } & \multicolumn{3}{|c|}{18 MAP } \\
\hline & & \multirow{2}{*}{$\begin{array}{r}\mathbf{2 0 1 5} \\
54.73 \mathrm{~g}\end{array}$} & \multirow{2}{*}{$\begin{array}{r}\mathbf{2 0 1 6} \\
55.82 \mathrm{~g}\end{array}$} & \multirow{2}{*}{$\begin{array}{r}\text { Mean } \\
55.28 \mathrm{~g} \\
\end{array}$} & \multirow{2}{*}{$\begin{array}{r}\mathbf{2 0 1 5} \\
60.48 \mathrm{f}\end{array}$} & \multirow{2}{*}{$62.3 \mathrm{e}^{2016}$} & \multirow{2}{*}{$\begin{array}{c}\text { Mean } \\
61.39 \mathrm{f} \\
\end{array}$} & \multirow{2}{*}{$\begin{array}{r}\mathbf{2 0 1 5} \\
80.50 \mathrm{f}\end{array}$} & \multirow{2}{*}{\begin{tabular}{|c|}
$\mathbf{2 0 1 6}$ \\
$79.27 f$ \\
\end{tabular}} & \multirow{2}{*}{$\begin{array}{c}\text { Mean } \\
79.89 \mathrm{f} \\
\end{array}$} \\
\hline $\mathrm{T}_{1}$ & $\mathrm{~A}_{0} \mathrm{~B}_{0} \mathrm{C}_{0}$ & & & & & & & & & \\
\hline$T_{2}$ & $\mathrm{~A}_{0} \mathrm{~B}_{0} \mathrm{C}_{1}$ & $61.87 \mathrm{ef}$ & $63.78 \mathrm{e}$ & $62.83 \mathrm{e}$ & $69.21 \mathrm{ef}$ & $77.87 \mathrm{cde}$ & 73.54def & $88.96 \mathrm{ef}$ & $88.1 \mathrm{ef}$ & $88.53 \mathrm{ef}$ \\
\hline $\mathrm{T}_{3}$ & $\mathrm{~A}_{0} \mathrm{~B}_{1} \mathrm{C}_{0}$ & $58.18 \mathrm{fg}$ & $58.83 \mathrm{f}$ & $58.51 \mathrm{f}$ & $71.25 \mathrm{def}$ & $73.13 \mathrm{de}$ & $72.19 \mathrm{ef}$ & $81.22 \mathrm{f}$ & $82.3 \mathrm{f}$ & $81.76 \mathrm{f}$ \\
\hline $\mathrm{T}_{4}$ & $\mathrm{~A}_{0} \mathrm{~B}_{1} \mathrm{C}_{1}$ & $62.07 \mathrm{e}$ & $64.29 \mathrm{e}$ & $63.18 \mathrm{e}$ & $68.56 \mathrm{ef}$ & 79.3 bcde & $73.93 \mathrm{def}$ & 90.30ef & 90.3def & 90.30ef \\
\hline $\mathrm{T}_{5}$ & $\mathrm{~A}_{0} \mathrm{~B}_{2} \mathrm{C}_{0}$ & $59.01 \mathrm{ef}$ & $59.98 \mathrm{f}$ & $59.50 \mathrm{f}$ & $73.52 \mathrm{cdef}$ & $74.97 \mathrm{cde}$ & $74.25 \mathrm{def}$ & $83.79 f$ & $84.5 \mathrm{f}$ & $84.15 \mathrm{f}$ \\
\hline $\mathrm{T}_{6}$ & $\mathrm{~A}_{0} \mathrm{~B}_{2} \mathrm{C}_{1}$ & $62.38 \mathrm{e}$ & $65.41 \mathrm{e}$ & $63.90 \mathrm{e}$ & $69.53 \mathrm{ef}$ & 80.13 bcde & $74.83 \mathrm{cdef}$ & 92.08def & 90.73def & 91.41def \\
\hline $\mathrm{T}_{7}$ & $\mathrm{~A}_{1} \mathrm{~B}_{0} \mathrm{C}_{0}$ & $72.11 \mathrm{~cd}$ & $71.18 \mathrm{~d}$ & $71.65 \mathrm{~d}$ & 80.63abcdef & 81.73abcde & 81.18bcdef & $101.87 \mathrm{cde}$ & $101.63 \mathrm{cde}$ & $101.75 \mathrm{cde}$ \\
\hline $\mathrm{T}_{8}$ & $\mathrm{~A}_{1} \mathrm{~B}_{0} \mathrm{C}_{1}$ & $75.73 b c$ & $74.81 b c$ & $75.27 b c$ & 87.01abcde & $88.17 \mathrm{abcd}$ & $87.59 \mathrm{abcde}$ & $106.96 \mathrm{bcd}$ & $108.23 \mathrm{abc}$ & $107.60 \mathrm{bcd}$ \\
\hline $\mathrm{T}_{9}$ & $\mathrm{~A}_{1} \mathrm{~B}_{1} \mathrm{C}_{0}$ & $76.66 \mathrm{~b}$ & $75.67 b$ & $76.17 b$ & 88.67abcde & $90.47 \mathrm{abcd}$ & 89.57 abcde & $107.54 \mathrm{bcd}$ & $110.4 \mathrm{abc}$ & $108.97 \mathrm{abc}$ \\
\hline $\mathrm{T}_{10}$ & $\mathrm{~A}_{1} \mathrm{~B}_{1} \mathrm{C}_{1}$ & $83.83 a$ & $83.77 \mathrm{a}$ & $83.80 \mathrm{a}$ & $98.33 \mathrm{ab}$ & $100.03 \mathrm{ab}$ & $99.18 \mathrm{ab}$ & $121.68 \mathrm{ab}$ & $120.57 \mathrm{ab}$ & $121.13 \mathrm{ab}$ \\
\hline $\mathrm{T}_{11}$ & $\mathrm{~A}_{1} \mathrm{~B}_{2} \mathrm{C}_{0}$ & $77.17 \mathrm{~b}$ & $76.12 b$ & $76.65 b$ & 91.31 abcd & 92.93abcd & 92.12adcde & $113.82 \mathrm{abc}$ & $111.9 \mathrm{abc}$ & $112.86 \mathrm{abc}$ \\
\hline$T_{12}$ & $\mathrm{~A}_{1} \mathrm{~B}_{2} \mathrm{C}_{1}$ & $85.84 \mathrm{a}$ & $85.11 \mathrm{a}$ & $85.48 \mathrm{a}$ & $101.42 \mathrm{a}$ & $102.83 \mathrm{a}$ & $102.13 \mathrm{a}$ & $125.06 \mathrm{a}$ & $123.33 \mathrm{a}$ & $124.20 \mathrm{a}$ \\
\hline $\mathrm{T}_{13}$ & $\mathrm{~A}_{2} \mathrm{~B}_{0} \mathrm{C}_{0}$ & $71.98 \mathrm{~d}$ & $69.90 \mathrm{~d}$ & $70.94 d$ & $78.73 \mathrm{bcdef}$ & $80.27 \mathrm{bcde}$ & $79.50 \mathrm{bcdef}$ & $101.57 \mathrm{cde}$ & $101.57 \mathrm{cde}$ & $101.57 \mathrm{cde}$ \\
\hline $\mathrm{T}_{14}$ & $\mathrm{~A}_{2} \mathrm{~B}_{0} \mathrm{C}_{1}$ & $77.02 \mathrm{~b}$ & $76.88 \mathrm{~b}$ & $76.95 b$ & 85.90abcde & 88.87abcd & 87.39abcde & $109.77 \mathrm{abc}$ & $109.57 \mathrm{abc}$ & $109.67 \mathrm{abc}$ \\
\hline$\frac{14}{T_{15}}$ & $\mathrm{~A}_{2} \mathrm{~B}_{1} \mathrm{C}_{0}$ & $72.65 \mathrm{~cd}$ & $72.08 \mathrm{~cd}$ & $72.37 \mathrm{~cd}$ & 82.37 abcde & $85.27 \mathrm{abcd}$ & 83.82abcde & $103.76 \mathrm{cde}$ & $105.03 \mathrm{bcd}$ & $104.40 \mathrm{cde}$ \\
\hline $\mathrm{T}_{16}$ & $\mathrm{~A}_{2} \mathrm{~B}_{1} \mathrm{C}_{1}$ & $83.13 \mathrm{a}$ & $82.69 \mathrm{a}$ & $82.91 \mathrm{a}$ & $93.03 \mathrm{abc}$ & $94.4 \mathrm{abc}$ & 93.72abcd & $111.53 \mathrm{abc}$ & $112.8 \mathrm{abc}$ & $112.17 \mathrm{abc}$ \\
\hline $\mathrm{T}_{17}$ & $\mathrm{~A}_{2} \mathrm{~B}_{2} \mathrm{C}_{0}$ & $73.60 \mathrm{bcd}$ & $70.69 d$ & $72.15 \mathrm{~d}$ & 85.24abcde & 84abcd & 84.62abcde & $103.27 \mathrm{cde}$ & 104.07bcde & $103.67 \mathrm{cde}$ \\
\hline $\mathrm{T}_{18}$ & $\mathrm{~A}_{2} \mathrm{~B}_{2} \mathrm{C}_{1}$ & $84.36 \mathrm{a}$ & $83.48 \mathrm{a}$ & $83.92 \mathrm{a}$ & $95.82 \mathrm{ab}$ & $94.43 \mathrm{abc}$ & $95.13 \mathrm{abc}$ & $115.27 \mathrm{abc}$ & $113.87 \mathrm{abc}$ & $114.57 \mathrm{abc}$ \\
\hline \multicolumn{2}{|c|}{ S.Em. $( \pm)$} & 1.29 & 1.04 & 1.05 & 7.27 & 7.40 & 7.26 & 5.57 & 5.86 & 5.70 \\
\hline \multicolumn{2}{|c|}{ L.S.D. $(\mathrm{P} \leq 0.05)$} & 3.72 & 2.99 & 3.01 & 20.91 & 21.26 & 20.86 & 16.01 & 16.83 & 16.39 \\
\hline
\end{tabular}

MAP-Month after planting

**Means with the same letter are not significantly different 
Table.4 Effect of Nutrient management on Number of leaves

\begin{tabular}{|c|c|c|c|c|c|c|c|c|c|}
\hline \multirow[t]{2}{*}{ Treatments } & \multicolumn{3}{|c|}{3 MAP } & \multicolumn{3}{|c|}{6 MAP } & \multicolumn{3}{|c|}{9 MAP } \\
\hline & 2015 & 2016 & Mean & 2015 & 2016 & Mean & 2015 & 2016 & Mean \\
\hline $\mathrm{A}_{0}$ & $12.06 \mathrm{~b}$ & $11.17 \mathrm{~b}$ & $11.61 \mathrm{~b}$ & $20.00 \mathrm{~b}$ & $20.23 b$ & $20.11 \mathrm{~b}$ & $27.87 \mathrm{~b}$ & $28.36 \mathrm{~b}$ & $28.12 b$ \\
\hline $\mathrm{A}_{1}$ & $15.77 \mathrm{a}$ & $16.46 \mathrm{a}$ & $16.11 \mathrm{a}$ & $25.84 \mathrm{a}$ & $26.84 a$ & $26.34 \mathrm{a}$ & $34.69 \mathrm{a}$ & $35.46 \mathrm{a}$ & $35.08 \mathrm{a}$ \\
\hline $\mathrm{A}_{2}$ & $15.55 \mathrm{a}$ & $15.58 \mathrm{a}$ & $15.56 \mathrm{a}$ & $25.52 \mathrm{a}$ & $26.22 \mathrm{a}$ & $25.87 \mathrm{a}$ & $33.83 \mathrm{a}$ & $34.90 \mathrm{a}$ & $34.37 \mathrm{a}$ \\
\hline S.Em. ( \pm$)$ & 0.28 & 0.35 & 0.29 & 0.48 & 0.57 & 0.51 & 0.43 & 0.53 & 0.44 \\
\hline L.S.D(P $\leq 0.05)$ & 0.80 & 0.99 & 0.85 & 1.39 & 1.63 & 1.47 & 1.23 & 1.52 & 1.27 \\
\hline $\mathrm{B}_{0}$ & $13.73 \mathrm{~b}$ & $13.92 \mathrm{a}$ & $13.83 \mathrm{~b}$ & $22.71 \mathrm{~b}$ & $23.75 \mathrm{a}$ & $23.23 a$ & $31.09 \mathrm{~b}$ & $32.26 \mathrm{a}$ & $31.67 \mathrm{~b}$ \\
\hline $\mathrm{B}_{1}$ & $14.93 \mathrm{a}$ & $14.52 \mathrm{a}$ & $14.73 \mathrm{a}$ & $24.13 \mathrm{a}$ & $24.72 \mathrm{a}$ & $24.42 a$ & $32.44 \mathrm{a}$ & $33.11 \mathrm{a}$ & $32.78 \mathrm{ab}$ \\
\hline $\mathrm{B}_{2}$ & $14.71 \mathrm{a}$ & $14.77 \mathrm{a}$ & $14.74 \mathrm{a}$ & $24.52 \mathrm{a}$ & $24.82 \mathrm{a}$ & $24.67 \mathrm{a}$ & $32.86 \mathrm{a}$ & $33.36 \mathrm{a}$ & $33.11 \mathrm{a}$ \\
\hline S.Em. ( \pm$)$ & 0.28 & 0.35 & 0.29 & 0.48 & 0.57 & 0.57 & 0.43 & 0.53 & 0.44 \\
\hline L.S.D(P $\leq 0.05)$ & 0.80 & NS & 0.85 & 1.39 & NS & NS & 1.23 & NS & 1.27 \\
\hline $\mathrm{C}_{0}$ & $13.66 \mathrm{~b}$ & $13.82 \mathrm{~b}$ & $13.74 \mathrm{~b}$ & $23.10 \mathrm{~b}$ & $23.84 \mathrm{a}$ & $23.47 \mathrm{~b}$ & 31.36 & $32.27 \mathrm{~b}$ & $31.81 \mathrm{~b}$ \\
\hline $\mathrm{C}_{1}$ & $15.26 \mathrm{a}$ & $14.98 \mathrm{a}$ & $15.12 \mathrm{a}$ & $24.47 \mathrm{a}$ & $25.02 \mathrm{a}$ & $24.74 a$ & $32.90 \mathrm{a}$ & $33.55 \mathrm{a}$ & $33.22 \mathrm{a}$ \\
\hline S.Em. $( \pm)$ & 0.23 & 0.28 & 0.24 & 0.40 & 0.46 & 0.42 & 0.35 & 0.43 & 0.36 \\
\hline L.S.D(P $\leq 0.05)$ & 0.65 & 0.82 & 0.69 & 1.14 & NS & 1.20 & 1.00 & 1.24 & 1.04 \\
\hline
\end{tabular}

\begin{tabular}{|c|c|c|c|c|c|c|c|c|c|c|}
\hline \multirow{2}{*}{\multicolumn{2}{|c|}{$\begin{array}{l}\text { Treatments/ } \\
\text { Combination }\end{array}$}} & \multicolumn{3}{|c|}{3 MAP } & \multicolumn{3}{|c|}{6 MAP } & \multicolumn{3}{|c|}{9 MAP } \\
\hline & & 2015 & 2016 & Mean & 2015 & 2016 & Mean & 2015 & 2016 & Mean \\
\hline $\mathrm{T}_{1}$ & $\mathrm{~A}_{0} \mathrm{~B}_{0} \mathrm{C}_{0}$ & $10.63 \mathrm{~h}$ & $9.9 \mathrm{~d}$ & $10.27 \mathrm{f}$ & $18.30 \mathrm{f}$ & $18.9 \mathrm{c}$ & $18.60 \mathrm{e}$ & $25.83 \mathrm{e}$ & $26.5 \mathrm{~b}$ & $26.17 \mathrm{c}$ \\
\hline $\mathrm{T}_{2}$ & $\mathrm{~A}_{0} \mathrm{~B}_{0} \mathrm{C}_{1}$ & 12.40fgh & $11.57 \mathrm{~d}$ & 11.99def & $20.20 \mathrm{ef}$ & $20.57 b c$ & $20.39 \mathrm{e}$ & $28.33 \mathrm{e}$ & $28.8 \mathrm{~b}$ & $28.57 \mathrm{c}$ \\
\hline $\mathrm{T}_{3}$ & $\mathrm{~A}_{0} \mathrm{~B}_{1} \mathrm{C}_{0}$ & $11.60 \mathrm{gh}$ & $10.7 \mathrm{~d}$ & $11.15 \mathrm{ef}$ & $19.63 \mathrm{f}$ & $20.03 c$ & $19.83 \mathrm{e}$ & $27.57 \mathrm{e}$ & $28.07 \mathrm{~b}$ & $27.82 \mathrm{c}$ \\
\hline $\mathrm{T}_{4}$ & $\mathrm{~A}_{0} \mathrm{~B}_{1} \mathrm{C}_{1}$ & 13.50efg & $11.87 \mathrm{~cd}$ & $12.69 \mathrm{de}$ & $21.07 \mathrm{def}$ & $20.87 \mathrm{bc}$ & $20.97 \mathrm{cde}$ & $28.80 \mathrm{de}$ & $29 b$ & $28.90 \mathrm{c}$ \\
\hline $\mathrm{T}_{5}$ & $\mathrm{~A}_{0} \mathrm{~B}_{2} \mathrm{C}_{0}$ & $11.00 \mathrm{~h}$ & $11.07 \mathrm{~d}$ & $11.04 \mathrm{ef}$ & $20.23 \mathrm{ef}$ & $20.07 \mathrm{c}$ & $20.15 \mathrm{e}$ & $28.03 \mathrm{e}$ & $28.4 \mathrm{~b}$ & $28.22 \mathrm{c}$ \\
\hline $\mathrm{T}_{6}$ & $\mathrm{~A}_{0} \mathrm{~B}_{2} \mathrm{C}_{1}$ & $13.20 \mathrm{efg}$ & $11.93 \mathrm{~cd}$ & $12.57 \mathrm{de}$ & $20.57 \mathrm{def}$ & $20.93 b c$ & $20.75 \mathrm{de}$ & $28.67 \mathrm{e}$ & $29.4 b$ & $29.04 \mathrm{c}$ \\
\hline $\mathrm{T}_{7}$ & $\mathrm{~A}_{1} \mathrm{~B}_{0} \mathrm{C}_{0}$ & $14.43 \mathrm{cde}$ & $15.83 \mathrm{ab}$ & $15.13 \mathrm{abc}$ & $23.37 \mathrm{cde}$ & $25.47 \mathrm{a}$ & $24.42 \mathrm{abc}$ & $32.60 \mathrm{bc}$ & $34.07 \mathrm{a}$ & $33.34 \mathrm{~b}$ \\
\hline $\mathrm{T}_{8}$ & $\mathrm{~A}_{1} \mathrm{~B}_{0} \mathrm{C}_{1}$ & 15.50abcd & $16.03 \mathrm{ab}$ & $15.77 \mathrm{abc}$ & $24.90 \mathrm{abc}$ & $26.7 \mathrm{a}$ & $25.80 \mathrm{ab}$ & $33.87 \mathrm{abc}$ & $35.13 \mathrm{a}$ & $34.50 \mathrm{ab}$ \\
\hline $\mathrm{T}_{9}$ & $\mathrm{~A}_{1} \mathrm{~B}_{1} \mathrm{C}_{0}$ & 15.60abcd & $16.4 \mathrm{ab}$ & $16.00 \mathrm{ab}$ & $25.07 \mathrm{abc}$ & $26.4 \mathrm{a}$ & $25.74 \mathrm{ab}$ & $34.17 \mathrm{abc}$ & $34.97 \mathrm{a}$ & $34.57 \mathrm{ab}$ \\
\hline $\mathrm{T}_{10}$ & $\mathrm{~A}_{1} \mathrm{~B}_{1} \mathrm{C}_{1}$ & $17.03 \mathrm{a}$ & $17.03 \mathrm{a}$ & $17.03 \mathrm{a}$ & $27.63 \mathrm{a}$ & $27.7 \mathrm{a}$ & $27.67 \mathrm{ab}$ & $35.57 \mathrm{ab}$ & $36.07 \mathrm{a}$ & $35.82 \mathrm{ab}$ \\
\hline $\mathrm{T}_{11}$ & $\mathrm{~A}_{1} \mathrm{~B}_{2} \mathrm{C}_{0}$ & 15.47abcd & $16.47 \mathrm{ab}$ & $15.97 \mathrm{ab}$ & $26.53 \mathrm{abc}$ & $26.8 \mathrm{a}$ & $26.67 \mathrm{ab}$ & $35.33 \mathrm{ab}$ & $35.53 \mathrm{a}$ & $35.43 \mathrm{ab}$ \\
\hline $\mathrm{T}_{12}$ & $\mathrm{~A}_{1} \mathrm{~B}_{2} \mathrm{C}_{1}$ & $16.60 \mathrm{ab}$ & $16.97 \mathrm{a}$ & $16.79 \mathrm{ab}$ & $27.53 \mathrm{a}$ & $27.97 \mathrm{a}$ & $27.75 \mathrm{a}$ & $36.60 \mathrm{a}$ & $37 \mathrm{a}$ & $36.80 \mathrm{a}$ \\
\hline $\mathrm{T}_{13}$ & $\mathrm{~A}_{2} \mathrm{~B}_{0} \mathrm{C}_{0}$ & 13.70def & $14.03 \mathrm{bc}$ & $13.87 \mathrm{~cd}$ & $23.87 \mathrm{bcd}$ & $24.37 \mathrm{ab}$ & $24.12 \mathrm{bcd}$ & $31.80 \mathrm{~cd}$ & $33.7 \mathrm{a}$ & $32.75 b$ \\
\hline $\mathrm{T}_{14}$ & $\mathrm{~A}_{2} \mathrm{~B}_{0} \mathrm{C}_{1}$ & $15.73 \mathrm{abc}$ & $16.17 \mathrm{ab}$ & $15.95 \mathrm{ab}$ & $25.63 \mathrm{abc}$ & $26.5 \mathrm{a}$ & $26.07 \mathrm{ab}$ & $34.10 \mathrm{abc}$ & $35.33 \mathrm{a}$ & $34.72 \mathrm{ab}$ \\
\hline $\mathrm{T}_{15}$ & $\mathrm{~A}_{2} \mathrm{~B}_{1} \mathrm{C}_{0}$ & 15.00bcde & $14.6 \mathrm{ab}$ & $14.80 \mathrm{bc}$ & $25.53 \mathrm{abc}$ & $26.5 \mathrm{a}$ & $26.02 \mathrm{ab}$ & $33.47 b c$ & $34.8 \mathrm{a}$ & $34.14 \mathrm{ab}$ \\
\hline $\mathrm{T}_{16}$ & $\mathrm{~A}_{2} \mathrm{~B}_{1} \mathrm{C}_{1}$ & $16.87 \mathrm{ab}$ & $16.5 \mathrm{a}$ & $16.69 \mathrm{ab}$ & $25.83 a b c$ & $26.8 \mathrm{a}$ & $26.32 \mathrm{ab}$ & $35.07 \mathrm{ab}$ & $35.77 \mathrm{a}$ & $35.42 \mathrm{ab}$ \\
\hline $\mathrm{T}_{17}$ & $\mathrm{~A}_{2} \mathrm{~B}_{2} \mathrm{C}_{0}$ & 15.47abcd & $15.4 \mathrm{ab}$ & $15.44 \mathrm{abc}$ & $25.40 \mathrm{abc}$ & $26.07 \mathrm{a}$ & $25.74 \mathrm{ab}$ & $33.43 b c$ & $34.37 \mathrm{a}$ & $33.90 \mathrm{ab}$ \\
\hline $\mathrm{T}_{18}$ & $\mathrm{~A}_{2} \mathrm{~B}_{2} \mathrm{C}_{1}$ & $16.53 \mathrm{ab}$ & $16.77 \mathrm{a}$ & $16.65 \mathrm{ab}$ & $26.87 \mathrm{ab}$ & $27.1 \mathrm{a}$ & $26.99 \mathrm{ab}$ & $35.10 \mathrm{ab}$ & $35.43 \mathrm{a}$ & $35.27 \mathrm{ab}$ \\
\hline \multicolumn{2}{|c|}{ S.Em. $( \pm)$} & 0.68 & 0.85 & 0.72 & 1.19 & 1.39 & 1.26 & 1.05 & 1.30 & 1.08 \\
\hline \multicolumn{2}{|c|}{ L.S.D. $(\mathrm{P} \leq 0.05)$} & 1.96 & 2.45 & 2.07 & 3.98 & 3.98 & 3.61 & 3.01 & 3.73 & 3.11 \\
\hline
\end{tabular}


Table.4 Effect of Nutrient management on Number of leaves (contd....)

\begin{tabular}{|c|c|c|c|c|c|c|c|c|c|}
\hline \multirow[t]{2}{*}{ Treatments } & \multicolumn{3}{|c|}{12 MAP } & \multicolumn{3}{|c|}{15 MAP } & \multicolumn{3}{|c|}{18 MAP } \\
\hline & 2015 & 2016 & Mean & 2015 & 2016 & Mean & 2015 & 2016 & Mean \\
\hline $\mathrm{A}_{0}$ & $32.28 \mathrm{c}$ & $34.48 \mathrm{~b}$ & $33.88 \mathrm{~b}$ & $37.08 \mathrm{~b}$ & $39.67 \mathrm{~b}$ & $38.37 \mathrm{~b}$ & $41.11 \mathrm{~b}$ & $41.78 \mathrm{~b}$ & $44.44 \mathrm{~b}$ \\
\hline $\mathrm{A}_{1}$ & $42.28 \mathrm{a}$ & $42.38 \mathrm{a}$ & $42.33 a$ & $48.53 a$ & $48.89 \mathrm{a}$ & $48.71 \mathrm{a}$ & $52.47 \mathrm{a}$ & $53.00 \mathrm{a}$ & $52.74 \mathrm{a}$ \\
\hline $\mathrm{A}_{2}$ & $39.73 b$ & $39.38 \mathrm{a}$ & $39.55 a$ & $46.04 \mathrm{a}$ & $45.67 \mathrm{a}$ & $45.85 \mathrm{a}$ & $49.47 \mathrm{a}$ & $49.50 \mathrm{a}$ & $49.48 \mathrm{a}$ \\
\hline S.Em. ( \pm$)$ & 0.85 & 1.26 & 1.01 & 1.49 & 1.75 & 1.60 & 1.44 & 1.60 & 1.51 \\
\hline L.S.D(P $\leq 0.05)$ & 2.45 & 3.61 & 2.90 & 4.29 & 5.04 & 4.59 & 4.11 & 4.59 & 4.34 \\
\hline $\mathrm{B}_{0}$ & $36.13 b$ & $36.72 \mathrm{a}$ & $36.43 \mathrm{~b}$ & $40.76 \mathrm{a}$ & $42.39 \mathrm{a}$ & $41.58 \mathrm{a}$ & $45.26 \mathrm{a}$ & $45.72 \mathrm{a}$ & $45.49 \mathrm{a}$ \\
\hline $\mathrm{B}_{1}$ & $38.99 \mathrm{a}$ & $39.34 \mathrm{a}$ & $39.17 \mathrm{ab}$ & $44.86 \mathrm{ab}$ & $45.56 \mathrm{a}$ & $45.21 \mathrm{a}$ & $48.57 \mathrm{a}$ & $48.89 \mathrm{a}$ & $48.73 a$ \\
\hline $\mathrm{B}_{2}$ & $40.16 \mathrm{a}$ & $40.17 \mathrm{a}$ & $40.17 \mathrm{a}$ & $46.03 a$ & $46.28 \mathrm{a}$ & $46.15 a$ & $49.22 \mathrm{a}$ & $49.67 \mathrm{a}$ & $49.44 a$ \\
\hline S.Em. $( \pm)$ & 0.85 & 1.26 & 1.01 & 1.49 & 1.75 & 1.60 & 1.44 & 1.60 & 1.51 \\
\hline L.S.D(P $\leq 0.05)$ & 2.45 & NS & 2.90 & 4.29 & NS & NS & NS & NS & NS \\
\hline $\mathrm{C}_{0}$ & $36.63 b$ & $36.68 \mathrm{~b}$ & $36.65 b$ & $41.26 \mathrm{~b}$ & $42.67 \mathrm{~b}$ & $41.97 \mathrm{~b}$ & $45.18 \mathrm{~b}$ & $45.78 \mathrm{~b}$ & $45.48 \mathrm{~b}$ \\
\hline $\mathrm{C}_{1}$ & $40.23 a$ & $40.81 \mathrm{a}$ & $40.52 a$ & $46.50 \mathrm{a}$ & $46.82 a$ & $46.66 \mathrm{a}$ & $50.19 \mathrm{a}$ & $50.41 \mathrm{a}$ & $50.30 \mathrm{a}$ \\
\hline S.Em. $( \pm)$ & $\mathbf{0 . 7 0}$ & 1.03 & 0.82 & 1.22 & 1.43 & 1.30 & 1.18 & 1.30 & 1.23 \\
\hline L.S.D(P $\leq 0.05)$ & 2.00 & 2.95 & 2.36 & 3.50 & 4.11 & 3.75 & 3.88 & 3.75 & 3.54 \\
\hline
\end{tabular}

\begin{tabular}{|c|c|c|c|c|c|c|c|c|c|c|}
\hline \multirow{2}{*}{\multicolumn{2}{|c|}{$\begin{array}{l}\text { Treatments/ } \\
\text { Combination }\end{array}$}} & \multicolumn{3}{|c|}{12 MAP } & \multicolumn{3}{|c|}{15 MAP } & \multicolumn{3}{|c|}{18 MAP } \\
\hline & & \multirow{2}{*}{$\begin{array}{r}\mathbf{2 0 1 5} \\
33.03 \mathrm{~g}\end{array}$} & \multirow{2}{*}{\begin{tabular}{|c|}
\multicolumn{1}{|c|}{$\mathbf{2 0 1 6}$} \\
$32.63 \mathrm{e}$ \\
\end{tabular}} & \multirow{2}{*}{\begin{tabular}{|l} 
Mean \\
$32.83 \mathrm{f}$ \\
\end{tabular}} & \multirow{2}{*}{\begin{tabular}{|l}
$\mathbf{2 0 1 5}$ \\
$34.27 \mathrm{~g}$ \\
\end{tabular}} & \multirow{2}{*}{\begin{tabular}{|c|}
$\mathbf{2 0 1 6}$ \\
$38.00 \mathrm{~d}$
\end{tabular}} & \multirow{2}{*}{$\begin{array}{l}\text { Mean } \\
36.14 \mathrm{f}\end{array}$} & \multirow{2}{*}{$\begin{array}{c}\mathbf{2 0 1 5} \\
39.20 \mathrm{e} \\
\end{array}$} & 2016 & \multirow{2}{*}{$\begin{array}{c}\text { Mean } \\
39.27 \mathrm{a} \\
\end{array}$} \\
\hline $\mathrm{T}_{1}$ & $\mathrm{~A}_{0} \mathrm{~B}_{0} \mathrm{C}_{0}$ & & & & & & & & $39.33 \mathrm{~d}$ & \\
\hline $\mathrm{T}_{2}$ & $\mathrm{~A}_{0} \mathrm{~B}_{0} \mathrm{C}_{1}$ & $33.40 \mathrm{~g}$ & $35.67 \mathrm{cde}$ & $34.54 \mathrm{ef}$ & $38.20 \mathrm{efg}$ & $40.33 \mathrm{~cd}$ & $39.27 \mathrm{def}$ & $42.27 \mathrm{de}$ & $43.00 \mathrm{~cd}$ & $42.64 \mathrm{cde}$ \\
\hline $\mathrm{T}_{3}$ & $\mathrm{~A}_{0} \mathrm{~B}_{1} \mathrm{C}_{0}$ & $33.10 \mathrm{~g}$ & 34.00de & $33.55 \mathrm{ef}$ & $36.30 \mathrm{fg}$ & $39.00 \mathrm{~cd}$ & $37.65 \mathrm{ef}$ & $39.03 \mathrm{e}$ & $40.33 \mathrm{~d}$ & $39.68 \mathrm{de}$ \\
\hline $\mathrm{T}_{4}$ & $\mathrm{~A}_{0} \mathrm{~B}_{1} \mathrm{C}_{1}$ & $33.47 \mathrm{fg}$ & $34.80 \mathrm{cde}$ & $34.17 \mathrm{ef}$ & 38.40 efg & $40.33 \mathrm{~cd}$ & 39.37def & 42.73de & $43.00 \mathrm{~cd}$ & $42.87 \mathrm{cde}$ \\
\hline $\mathrm{T}_{5}$ & $\mathrm{~A}_{0} \mathrm{~B}_{2} \mathrm{C}_{0}$ & $33.13 \mathrm{~g}$ & \begin{tabular}{|l|}
$34.17 \mathrm{de}$ \\
\end{tabular} & $33.65 \mathrm{ef}$ & $36.37 \mathrm{fg}$ & $39.67 \mathrm{~cd}$ & $38.02 \mathrm{ef}$ & $40.17 \mathrm{e}$ & $41.60 \mathrm{~cd}$ & $40.92 \mathrm{de}$ \\
\hline $\mathrm{T}_{6}$ & $\mathrm{~A}_{0} \mathrm{~B}_{2} \mathrm{C}_{1}$ & $33.53 \mathrm{fg}$ & $35.53 \mathrm{cde}$ & $34.53 \mathrm{ef}$ & $38.93 \mathrm{efg}$ & $40.67 \mathrm{~cd}$ & 39.80def & $43.23 \mathrm{cde}$ & $43.33 \mathrm{~cd}$ & $43.28 \mathrm{cde}$ \\
\hline $\mathrm{T}_{7}$ & $\mathrm{~A}_{1} \mathrm{~B}_{0} \mathrm{C}_{0}$ & $36.33 \mathrm{efg}$ & $36.77 \mathrm{cde}$ & 36.55def & 42.77cdefg & 43.00abcd & 42.89cdef & $45.37 \mathrm{bcde}$ & $46.33 \mathrm{bcd}$ & $45.85 \mathrm{bcde}$ \\
\hline $\mathrm{T}_{8}$ & $\mathrm{~A}_{1} \mathrm{~B}_{0} \mathrm{C}_{1}$ & 38.30defg & 38.63bcde & $38.47 \mathrm{cdef}$ & 43.07cdefg & 45.00abcd & 44.04bcdef & 49.00abcde & 49.67abcd & 49.34abde \\
\hline $\mathrm{T}_{9}$ & $\mathrm{~A}_{1} \mathrm{~B}_{1} \mathrm{C}_{0}$ & $40.40 \mathrm{cde}$ & 40.6abcde & 40.50bcde & 46.27abcdef & 47.33abcd & 46.80abcdef & 51.93abcd & $52.00 \mathrm{abc}$ & $51.97 \mathrm{babc}$ \\
\hline $\mathrm{T}_{10}$ & $\mathrm{~A}_{1} \mathrm{~B}_{1} \mathrm{C}_{1}$ & $47.17 \mathrm{ab}$ & $47.47 \mathrm{ab}$ & $47.32 \mathrm{ab}$ & $54.90 \mathrm{ab}$ & $54.67 \mathrm{ab}$ & $54.79 \mathrm{ab}$ & $57.77 \mathrm{a}$ & $58.33 \mathrm{a}$ & $58.05 \mathrm{a}$ \\
\hline $\mathrm{T}_{11}$ & $\mathrm{~A}_{1} \mathrm{~B}_{2} \mathrm{C}_{0}$ & 42.00bcde & 41.53abcd & $41.77 \mathrm{bcd}$ & 48.00abcde & 48.00abcd & 48.00abcde & 52.03abcd & $52.67 \mathrm{abc}$ & $52.35 \mathrm{abc}$ \\
\hline $\mathrm{T}_{12}$ & $\mathrm{~A}_{1} \mathrm{~B}_{2} \mathrm{C}_{1}$ & $49.47 \mathrm{a}$ & $49.27 \mathrm{a}$ & $49.37 \mathrm{a}$ & $56.17 \mathrm{a}$ & $55.33 \mathrm{a}$ & $55.75 \mathrm{a}$ & $58.73 \mathrm{a}$ & $59.00 \mathrm{a}$ & $58.87 \mathrm{a}$ \\
\hline $\mathrm{T}_{13}$ & $\mathrm{~A}_{2} \mathrm{~B}_{0} \mathrm{C}_{0}$ & $36.27 \mathrm{efg}$ & $36.27 \mathrm{cde}$ & 36.27def & 41.10defg & $42.67 \mathrm{bcd}$ & 41.89cdef & $45.20 \mathrm{bcde}$ & $46.00 \mathrm{bcd}$ & $45.60 \mathrm{bcde}$ \\
\hline $\mathrm{T}_{14}$ & $\mathrm{~A}_{2} \mathrm{~B}_{0} \mathrm{C}_{1}$ & $39.43 \mathrm{cdef}$ & 40.37bcde & 39.90cdef & $45.17 \mathrm{bcdef}$ & 45.33abcd & 45.25abcdef & 50.50abcd & 50.00abcd & $50.25 \mathrm{abcd}$ \\
\hline $\mathrm{T}_{15}$ & $\mathrm{~A}_{2} \mathrm{~B}_{1} \mathrm{C}_{0}$ & 37.33defg & $37.27 \mathrm{cde}$ & 37.30cdef & 42.67cdefg & 43.33abcd & 43.00cdef & $47.00 \mathrm{bcde}$ & $47.00 \mathrm{bcd}$ & $47.00 \mathrm{bcde}$ \\
\hline $\mathrm{T}_{16}$ & $\mathrm{~A}_{2} \mathrm{~B}_{1} \mathrm{C}_{1}$ & 42.50bcd & 41.83abcd & 42.17bcd & 50.60abcd & 48.67abcd & 49.64abcd & $52.97 \mathrm{abc}$ & $52.67 \mathrm{abc}$ & $52.82 \mathrm{abc}$ \\
\hline $\mathrm{T}_{17}$ & $\mathrm{~A}_{2} \mathrm{~B}_{2} \mathrm{C}_{0}$ & 38.03defg & 36.90cde & $37.47 \mathrm{cde}$ & 43.63cdefg & 43.00abcd & 43.32cdef & $46.67 \mathrm{bcde}$ & $46.67 \mathrm{bcd}$ & $46.67 \mathrm{bcde}$ \\
\hline $\mathrm{T}_{18}$ & $\mathrm{~A}_{2} \mathrm{~B}_{2} \mathrm{C}_{1}$ & $44.80 \mathrm{abc}$ & 43.63abc & $44.22 \mathrm{abc}$ & $53.07 \mathrm{abc}$ & $51.00 \mathrm{abc}$ & $52.04 \mathrm{abc}$ & $54.47 \mathrm{ab}$ & $54.67 \mathrm{ab}$ & $54.57 \mathrm{ab}$ \\
\hline \multicolumn{2}{|c|}{ S.Em. $( \pm)$} & 2.09 & \begin{tabular}{|l|}
3.08 \\
\end{tabular} & 2.47 & 3.66 & 4.29 & 3.91 & 3.53 & 3.91 & 3.7 \\
\hline \multicolumn{2}{|c|}{ L.S.D. $(\mathrm{P} \leq 0.05)$} & 5.99 & 8.85 & 7.10 & 10.51 & 12.34 & 11.24 & 10.13 & 11.24 & 10.63 \\
\hline
\end{tabular}


Table.5 Effect of Nutrient management on D-leaf length (cm)

\begin{tabular}{|c|c|c|c|c|c|c|c|c|c|}
\hline \multirow[t]{2}{*}{ Treatments } & \multicolumn{3}{|c|}{3 MAP } & \multicolumn{3}{|c|}{6 MAP } & \multicolumn{3}{|c|}{9 MAP } \\
\hline & 2015 & 2016 & Mean & 2015 & 2016 & Mean & 2015 & 2016 & Mean \\
\hline $\mathrm{A}_{0}$ & $20.02 b$ & $19.71 \mathrm{~b}$ & $19.86 \mathrm{~b}$ & $24.62 \mathrm{~b}$ & $25.24 \mathrm{~b}$ & $24.93 b$ & $29.79 \mathrm{c}$ & $32.85 \mathrm{~b}$ & $31.32 \mathrm{c}$ \\
\hline $\mathrm{A}_{1}$ & $22.54 \mathrm{a}$ & $22.47 \mathrm{a}$ & $22.64 a$ & $27.80 \mathrm{a}$ & $28.04 a$ & $27.92 a$ & $34.68 \mathrm{a}$ & $36.01 \mathrm{a}$ & $35.35 \mathrm{a}$ \\
\hline $\mathrm{A}_{2}$ & $22.51 \mathrm{a}$ & $22.40 \mathrm{a}$ & $22.47 \mathrm{a}$ & $27.31 \mathrm{a}$ & $27.69 \mathrm{a}$ & $27.50 \mathrm{a}$ & $34.03 \mathrm{~b}$ & $35045 \mathrm{a}$ & $34.74 \mathrm{~b}$ \\
\hline S.Em. ( $( \pm)$ & 0.33 & 0.27 & 0.29 & 0.31 & 0.27 & 0.28 & 0.21 & 0.26 & 0.20 \\
\hline L.S.D(P $\leq 0.05)$ & 0.94 & 0.78 & 0.84 & 0.90 & 0.78 & 0.81 & 0.60 & 0.75 & 0.58 \\
\hline $\mathrm{B}_{0}$ & $21.42 \mathrm{ab}$ & $21.15 \mathrm{a}$ & $21.29 \mathrm{a}$ & $26.45 \mathrm{a}$ & $26.46 \mathrm{~b}$ & $26.45 \mathrm{a}$ & $32.22 \mathrm{~b}$ & $34.07 \mathrm{~b}$ & $33.14 \mathrm{~b}$ \\
\hline $\mathrm{B}_{1}$ & $21.29 \mathrm{ab}$ & $21.82 \mathrm{a}$ & $21.56 \mathrm{a}$ & $26.52 a$ & $27.20 \mathrm{ab}$ & $26.86 a$ & $33.01 \mathrm{a}$ & $35.00 \mathrm{a}$ & $34.01 \mathrm{a}$ \\
\hline $\mathrm{B}_{2}$ & $22.36 \mathrm{a}$ & $21.87 \mathrm{a}$ & $22.12 \mathrm{a}$ & $26.78 \mathrm{a}$ & $27.31 \mathrm{a}$ & $27.05 \mathrm{a}$ & $33.27 \mathrm{a}$ & $35.24 \mathrm{a}$ & $34.26 \mathrm{a}$ \\
\hline S.Em. $( \pm)$ & 0.33 & 0.27 & 0.29 & 0.31 & 0.27 & 0.28 & 0.21 & 0.26 & 0.20 \\
\hline L.S.D(P $\leq 0.05)$ & 0.94 & NS & NS & NS & 0.78 & NS & 0.60 & 0.75 & 0.58 \\
\hline $\mathrm{C}_{0}$ & $21.21 \mathrm{~b}$ & $21.22 \mathrm{~b}$ & $21.22 \mathrm{~b}$ & $26.21 \mathrm{~b}$ & $26.63 b$ & $26.42 b$ & $32.18 \mathrm{~b}$ & $34.11 \mathrm{~b}$ & $33.15 b$ \\
\hline $\mathrm{C}_{1}$ & $22.16 \mathrm{a}$ & $22.01 \mathrm{a}$ & $22.09 \mathrm{a}$ & $26.95 \mathrm{a}$ & $27.35 \mathrm{a}$ & $27.15 \mathrm{a}$ & $32.49 \mathrm{a}$ & $35.43 a$ & $34.46 \mathrm{a}$ \\
\hline S.Em. $( \pm)$ & 0.27 & 0.22 & 0.24 & 0.26 & 0.22 & 0.23 & 0.17 & 0.21 & 0.17 \\
\hline L.S.D(P $\leq 0.05)$ & 0.77 & 0.64 & 0.69 & 0.73 & 0.63 & 0.66 & 0.49 & 0.62 & 0.48 \\
\hline
\end{tabular}

\begin{tabular}{|c|c|c|c|c|c|c|c|c|c|c|}
\hline \multirow{2}{*}{\multicolumn{2}{|c|}{$\begin{array}{l}\text { Treatments/ } \\
\text { Combination }\end{array}$}} & \multicolumn{3}{|c|}{3 МАР } & \multicolumn{3}{|c|}{6 MAP } & \multicolumn{3}{|c|}{9 MAP } \\
\hline & & \multirow{2}{*}{$\begin{array}{r}\mathbf{2 0 1 5} \\
17.43 \mathrm{~d}\end{array}$} & \multirow{2}{*}{$\begin{array}{r}\mathbf{2 0 1 6} \\
17.96 \mathrm{f}\end{array}$} & \multirow{2}{*}{$\begin{array}{l}\text { Mean } \\
17.70 \mathrm{f}\end{array}$} & \multirow{2}{*}{$\begin{array}{r}\mathbf{2 0 1 5} \\
23.01 \mathrm{~g}\end{array}$} & 2016 & \multirow{2}{*}{$\begin{array}{l}\text { Mean } \\
23.31 \mathrm{f}\end{array}$} & \multirow{2}{*}{$\begin{array}{r}\mathbf{2 0 1 5} \\
28.62 \mathrm{i}\end{array}$} & 2016 & \multirow{2}{*}{$\begin{array}{r}\text { Mean } \\
29.45 \mathrm{~h}\end{array}$} \\
\hline $\mathrm{T}_{1}$ & $\mathrm{~A}_{0} \mathrm{~B}_{0} \mathrm{C}_{0}$ & & & & & $23.60 \mathrm{f}$ & & & $30.28 \mathrm{~h}$ & \\
\hline $\mathrm{T}_{2}$ & $\mathrm{~A}_{0} \mathrm{~B}_{0} \mathrm{C}_{1}$ & $21.66 \mathrm{abc}$ & $20.26 \mathrm{cde}$ & 20.96bcde & $24.81 \mathrm{efg}$ & $25.48 \mathrm{cdef}$ & $25.15 \mathrm{ef}$ & $30.10 \mathrm{gh}$ & $33.74 \mathrm{efg}$ & $31.92 \mathrm{efg}$ \\
\hline $\mathrm{T}_{3}$ & $\mathrm{~A}_{0} \mathrm{~B}_{1} \mathrm{C}_{0}$ & $18.33 \mathrm{~d}$ & $19.57 \mathrm{ef}$ & $18.95 \mathrm{ef}$ & $25.25 \mathrm{def}$ & $25.26 \mathrm{ef}$ & $25.26 \mathrm{ef}$ & $29.35 \mathrm{hi}$ & $32.17 \mathrm{~g}$ & $30.76 \mathrm{gh}$ \\
\hline $\mathrm{T}_{4}$ & $\mathrm{~A}_{0} \mathrm{~B}_{1} \mathrm{C}_{1}$ & $19.69 \mathrm{~cd}$ & 20.31 bcde & $20.00 \mathrm{de}$ & $25.56 \mathrm{cdef}$ & $25.57 \mathrm{cde}$ & $25.57 \mathrm{de}$ & $30.29 \mathrm{gh}$ & $33.87 \mathrm{efg}$ & $32.08 \mathrm{efg}$ \\
\hline $\mathrm{T}_{5}$ & $\mathrm{~A}_{0} \mathrm{~B}_{2} \mathrm{C}_{0}$ & $22.05 \mathrm{ab}$ & $20.02 \mathrm{e}$ & $21.04 \mathrm{bcd}$ & $24.95 \mathrm{defg}$ & $25.42 \mathrm{def}$ & $25.19 \mathrm{ef}$ & 29.49ghi & $33.07 \mathrm{fg}$ & $31.28 \mathrm{efg}$ \\
\hline $\mathrm{T}_{6}$ & $\mathrm{~A}_{0} \mathrm{~B}_{2} \mathrm{C}_{1}$ & $20.94 b c$ & $20.16 \mathrm{de}$ & $20.55 \mathrm{cde}$ & $24.16 \mathrm{fg}$ & 26.08bcde & $25.12 \mathrm{ef}$ & $30.90 \mathrm{~g}$ & 33.97defg & $32.44 \mathrm{ef}$ \\
\hline $\mathrm{T}_{7}$ & $\mathrm{~A}_{1} \mathrm{~B}_{0} \mathrm{C}_{0}$ & $21.38 \mathrm{abc}$ & $22.19 \mathrm{ab}$ & 21.79abcd & $27.68 \mathrm{abc}$ & $27.34 \mathrm{abc}$ & 27.51abcd & 33.54def & $34.99 \mathrm{bcde}$ & $34.27 \mathrm{~cd}$ \\
\hline $\mathrm{T}_{8}$ & $\mathrm{~A}_{1} \mathrm{~B}_{0} \mathrm{C}_{1}$ & $23.61 \mathrm{a}$ & $22.34 \mathrm{a}$ & $22.98 \mathrm{ab}$ & $28.06 \mathrm{ab}$ & $27.60 \mathrm{ab}$ & $27.83 \mathrm{abc}$ & 34.06bcde & 35.38abcde & $34.72 \mathrm{bcd}$ \\
\hline $\mathrm{T}_{9}$ & $\mathrm{~A}_{1} \mathrm{~B}_{1} \mathrm{C}_{0}$ & $21.58 \mathrm{abc}$ & $22.64 \mathrm{a}$ & $22.11 \mathrm{abc}$ & 27.10abcd & $27.99 a$ & $27.55 \mathrm{abc}$ & 34.60abcde & $36.08 \mathrm{abc}$ & $35.34 \mathrm{abc}$ \\
\hline $\mathrm{T}_{10}$ & $\mathrm{~A}_{1} \mathrm{~B}_{1} \mathrm{C}_{1}$ & $22.51 \mathrm{ab}$ & $23.39 a$ & $22.95 \mathrm{ab}$ & $28.08 \mathrm{ab}$ & $28.65 \mathrm{a}$ & $28.37 \mathrm{abc}$ & $35.46 \mathrm{ab}$ & $36.51 \mathrm{ab}$ & $35.99 \mathrm{ab}$ \\
\hline $\mathrm{T}_{11}$ & $\mathrm{~A}_{1} \mathrm{~B}_{2} \mathrm{C}_{0}$ & $23.00 \mathrm{ab}$ & $22.43 \mathrm{a}$ & $22.72 \mathrm{ab}$ & $26.63 \mathrm{bcde}$ & $27.93 \mathrm{ab}$ & 27.28abcd & 34.58abcde & $36.04 \mathrm{abc}$ & $35.31 \mathrm{abc}$ \\
\hline $\mathrm{T}_{12}$ & $\mathrm{~A}_{1} \mathrm{~B}_{2} \mathrm{C}_{1}$ & $23.14 \mathrm{ab}$ & $23.42 \mathrm{a}$ & $23.28 \mathrm{a}$ & $29.27 \mathrm{a}$ & $28.68 \mathrm{a}$ & $28.98 \mathrm{a}$ & $35.82 \mathrm{a}$ & $37.05 \mathrm{a}$ & $36.44 a$ \\
\hline $\mathrm{T}_{13}$ & $\mathrm{~A}_{2} \mathrm{~B}_{0} \mathrm{C}_{0}$ & $22.52 \mathrm{ab}$ & $22.05 \mathrm{abcd}$ & $22.29 \mathrm{abc}$ & $28.36 \mathrm{ab}$ & 27.30abcd & $27.83 \mathrm{abc}$ & $32.39 \mathrm{f}$ & $34.25 \mathrm{cdef}$ & $33.32 \mathrm{de}$ \\
\hline $\mathrm{T}_{14}$ & $\mathrm{~A}_{2} \mathrm{~B}_{0} \mathrm{C}_{1}$ & $21.90 \mathrm{abc}$ & $22.12 \mathrm{abc}$ & 22.01abcd & 26.77bcde & $27.38 \mathrm{abc}$ & 27.08abcde & 34.60abcde & 35.76abcd & $35.18 \mathrm{abc}$ \\
\hline $\mathrm{T}_{15}$ & $\mathrm{~A}_{2} \mathrm{~B}_{1} \mathrm{C}_{0}$ & $23.02 \mathrm{ab}$ & $22.09 \mathrm{abc}$ & $22.56 a b c$ & 26.48bcde & $27.51 \mathrm{ab}$ & $27.00 \mathrm{bcde}$ & 33.37ef & $35.18 \mathrm{bcde}$ & $34.28 \mathrm{~cd}$ \\
\hline $\mathrm{T}_{16}$ & $\mathrm{~A}_{2} \mathrm{~B}_{1} \mathrm{C}_{1}$ & $22.59 \mathrm{ab}$ & $22.93 a$ & $22.76 \mathrm{ab}$ & $26.63 \mathrm{bcde}$ & $28.19 \mathrm{a}$ & 27.41abcd & 34.98abcd & $36.19 \mathrm{ab}$ & $35.59 \mathrm{abc}$ \\
\hline $\mathrm{T}_{17}$ & $\mathrm{~A}_{2} \mathrm{~B}_{2} \mathrm{C}_{0}$ & $21.59 \mathrm{abc}$ & 22.01abcd & 21.80abcd & $26.43 \mathrm{bcde}$ & 27.27abcd & $26.85 \mathrm{cde}$ & 33.69cdef & $34.91 \mathrm{bcdef}$ & $34.30 \mathrm{~cd}$ \\
\hline $\mathrm{T}_{18}$ & $\mathrm{~A}_{2} \mathrm{~B}_{2} \mathrm{C}_{1}$ & $23.41 \mathrm{a}$ & $23.20 \mathrm{a}$ & $23.31 \mathrm{a}$ & $29.22 \mathrm{a}$ & $28.46 \mathrm{a}$ & $28.84 \mathrm{ab}$ & $35.16 a b c$ & $36.41 \mathrm{ab}$ & $35.79 \mathrm{abc}$ \\
\hline \multicolumn{2}{|c|}{ S.Em. $( \pm)$} & 0.81 & 0.67 & 0.72 & 0.76 & 0.67 & 0.72 & 0.51 & 0.64 & 0.50 \\
\hline \multicolumn{2}{|c|}{ L.S.D. $(\mathrm{P} \leq 0.05)$} & 2.31 & 1.91 & 2.06 & 2.20 & 1.90 & 1.97 & 1.48 & 1.85 & 1.43 \\
\hline
\end{tabular}

MAP-Month after planting

**Means with the same letter are not significantly different 
Table.5 Effect of Nutrient management on D-leaf length (cm) (contd...)

\begin{tabular}{|c|c|c|c|c|c|c|c|c|c|}
\hline \multirow[t]{2}{*}{ Treatments } & \multicolumn{3}{|c|}{12 MAP } & \multicolumn{3}{|c|}{15 MAP } & \multicolumn{3}{|c|}{18 MAP } \\
\hline & 2015 & 2016 & Mean & 2015 & 2016 & Mean & 2015 & 2016 & Mean \\
\hline $\mathrm{A}_{0}$ & $37.64 \mathrm{~b}$ & $41.48 \mathrm{~b}$ & $39.56 \mathrm{~b}$ & $46.11 \mathrm{~b}$ & $48.31 \mathrm{~b}$ & $47.21 \mathrm{~b}$ & $54.44 \mathrm{~b}$ & $55.25 \mathrm{~b}$ & $54.85 b$ \\
\hline $\mathrm{A}_{1}$ & $44.90 \mathrm{a}$ & $45.39 \mathrm{a}$ & $45.15 \mathrm{a}$ & $51.56 \mathrm{a}$ & $51.86 \mathrm{a}$ & $51.71 \mathrm{a}$ & $59.18 \mathrm{a}$ & $60.35 \mathrm{a}$ & $59.77 \mathrm{a}$ \\
\hline $\mathrm{A}_{2}$ & $43.77 \mathrm{a}$ & $44.40 \mathrm{a}$ & $44.09 \mathrm{a}$ & $50.77 \mathrm{a}$ & $51.42 \mathrm{a}$ & $51.10 \mathrm{a}$ & $58.22 \mathrm{a}$ & $59.34 \mathrm{a}$ & $58.78 \mathrm{a}$ \\
\hline S.Em. ( \pm$)$ & 0.77 & 0.79 & 0.65 & 0.71 & 0.61 & 0.62 & 0.42 & 0.45 & 0.39 \\
\hline L.S.D(P $\leq 0.05)$ & 2.20 & 2.28 & 1.87 & 2.04 & 1.76 & 1.79 & 1.22 & 1.28 & 1.11 \\
\hline $\mathrm{B}_{0}$ & $40.80 \mathrm{~b}$ & $42.63 \mathrm{a}$ & $41.71 \mathrm{~b}$ & $48.58 \mathrm{a}$ & $49.40 \mathrm{~b}$ & $48.99 \mathrm{~b}$ & $55.91 \mathrm{~b}$ & $56.73 b$ & $56.32 \mathrm{~b}$ \\
\hline $\mathrm{B}_{1}$ & $42.39 \mathrm{ab}$ & $44.15 \mathrm{a}$ & $43.27 \mathrm{ab}$ & $49.62 \mathrm{a}$ & $50.67 \mathrm{ab}$ & $50.15 \mathrm{ab}$ & $57.88 \mathrm{a}$ & $58.74 a$ & $58.31 \mathrm{a}$ \\
\hline $\mathrm{B}_{2}$ & $43.13 a$ & $44.49 \mathrm{a}$ & $43.81 \mathrm{a}$ & $50.25 \mathrm{a}$ & $51.51 \mathrm{a}$ & $50.88 \mathrm{a}$ & $58.06 \mathrm{a}$ & $59.47 \mathrm{a}$ & $58.77 \mathrm{a}$ \\
\hline S.Em. $( \pm)$ & 0.77 & 0.79 & 0.65 & 0.71 & 0.61 & 0.62 & 0.42 & 0.45 & 0.39 \\
\hline L.S.D(P $\leq 0.05)$ & 2.20 & NS & 1.87 & NS & 1.76 & 1.79 & 1.22 & 1.28 & 1.11 \\
\hline $\mathrm{C}_{0}$ & $40.98 b$ & $42.76 \mathrm{~b}$ & $41.87 \mathrm{~b}$ & $48.73 a$ & $49.55 b$ & $49.15 b$ & $55.88 \mathrm{~b}$ & $56.99 \mathrm{~b}$ & $56.44 \mathrm{~b}$ \\
\hline $\mathrm{C}_{1}$ & $43.23 \mathrm{a}$ & $44.75 \mathrm{a}$ & $43.99 \mathrm{a}$ & $50.23 \mathrm{a}$ & $51.50 \mathrm{a}$ & $50.87 \mathrm{a}$ & $58.68 \mathrm{a}$ & $59.63 \mathrm{a}$ & $59.16 \mathrm{a}$ \\
\hline S.Em. ( $( \pm)$ & 0.63 & 0.64 & 0.53 & 0.58 & 0.50 & 0.51 & 0.35 & 0.36 & 0.31 \\
\hline L.S.D(P $\leq 0.05)$ & 1.80 & 1.86 & 1.53 & $\mathbf{N S}$ & 1.43 & 1.46 & 1.00 & 1.05 & 0.90 \\
\hline
\end{tabular}

\begin{tabular}{|c|c|c|c|c|c|c|c|c|c|c|}
\hline \multirow{2}{*}{\multicolumn{2}{|c|}{$\begin{array}{l}\text { Treatments/ } \\
\text { Combination }\end{array}$}} & \multicolumn{3}{|c|}{12 MAP } & \multicolumn{3}{|c|}{15 MAP } & \multicolumn{3}{|c|}{18 MAP } \\
\hline & & \multirow{2}{*}{\begin{tabular}{r|}
$\mathbf{2 0 1 5}$ \\
$36.72 \mathrm{~g}$ \\
\end{tabular}} & \multirow{2}{*}{$\begin{array}{r}\mathbf{2 0 1 6} \\
39.33 \mathrm{~d}\end{array}$} & \multirow{2}{*}{$\begin{array}{r}\text { Mean } \\
38.03 \mathrm{~h} \\
\end{array}$} & \multirow{2}{*}{\begin{tabular}{r|}
$\mathbf{2 0 1 5}$ \\
$44.59 \mathrm{~d}$ \\
\end{tabular}} & \multirow{2}{*}{$\begin{array}{c}\mathbf{2 0 1 6} \\
46.07 \mathrm{e} \\
\end{array}$} & \multirow{2}{*}{$\begin{array}{r}\text { Mean } \\
45.33 \mathrm{c} \\
\end{array}$} & \multirow{2}{*}{\begin{tabular}{r|}
$\mathbf{2 0 1 5}$ \\
$50.87 \mathrm{~g}$ \\
\end{tabular}} & \multirow{2}{*}{$\begin{array}{r}\mathbf{2 0 1 6} \\
51.30 \mathrm{~h}\end{array}$} & \multirow{2}{*}{$\begin{array}{r}\text { Mean } \\
51.09 \mathrm{i} \\
\end{array}$} \\
\hline $\mathrm{T}_{1}$ & $\mathrm{~A}_{0} \mathrm{~B}_{0} \mathrm{C}_{0}$ & & & & & & & & & \\
\hline $\mathrm{T}_{2}$ & $\mathrm{~A}_{0} \mathrm{~B}_{0} \mathrm{C}_{1}$ & $37.76 \mathrm{fg}$ & 42.07bcd & 39.92efgh & $46.33 \mathrm{bcd}$ & $48.77 \mathrm{de}$ & $47.55 \mathrm{bc}$ & 56.40def & $56.03 \mathrm{fg}$ & $56.22 \mathrm{fgh}$ \\
\hline $\mathrm{T}_{3}$ & $\mathrm{~A}_{0} \mathrm{~B}_{1} \mathrm{C}_{0}$ & $37.26 \mathrm{~g}$ & $41.2 \mathrm{~cd}$ & $39.23 \mathrm{gh}$ & $46.15 \mathrm{~cd}$ & $47.9 \mathrm{de}$ & $47.03 \mathrm{bc}$ & $54.00 \mathrm{f}$ & $54.70 \mathrm{~g}$ & $54.35 \mathrm{~h}$ \\
\hline $\mathrm{T}_{4}$ & $\mathrm{~A}_{0} \mathrm{~B}_{1} \mathrm{C}_{1}$ & $38.34 \mathrm{defg}$ & $42.33 \mathrm{bcd}$ & 40.34defgh & $47.0 \mathrm{bcd}$ & $49.2 \mathrm{cde}$ & $48.12 b c$ & 56.40def & 56.70efg & $56.55 \mathrm{efgh}$ \\
\hline $\mathrm{T}_{5}$ & $\mathrm{~A}_{0} \mathrm{~B}_{2} \mathrm{C}_{0}$ & $37.60 \mathrm{fg}$ & $41.5 \mathrm{~cd}$ & $39.55 \mathrm{gh}$ & 46.30bcd & 48.6de & $47.45 \mathrm{bc}$ & $54.75 \mathrm{ef}$ & $55.50 \mathrm{fg}$ & $55.13 \mathrm{gh}$ \\
\hline $\mathrm{T}_{6}$ & $\mathrm{~A}_{0} \mathrm{~B}_{2} \mathrm{C}_{1}$ & $38.19 \mathrm{efg}$ & $42.43 \mathrm{bcd}$ & 40.31defgh & $46.25 \mathrm{~cd}$ & $49.3 \mathrm{cde}$ & $47.78 \mathrm{bc}$ & $54.23 \mathrm{f}$ & $57.27 \mathrm{defg}$ & $55.75 \mathrm{fgh}$ \\
\hline $\mathrm{T}_{7}$ & $\mathrm{~A}_{1} \mathrm{~B}_{0} \mathrm{C}_{0}$ & 42.68bcdef & 42.93abcd & 42.81bcdefg & 49.93abc & $49.93 \mathrm{cde}$ & $49.93 \mathrm{ab}$ & 55.39ef & 57.50cdefg & $56.45 \mathrm{fgh}$ \\
\hline $\mathrm{T}_{8}$ & $\mathrm{~A}_{1} \mathrm{~B}_{0} \mathrm{C}_{1}$ & 43.58abcd & 44.33abcd & 43.96abcdef & $50.45 \mathrm{abc}$ & 50.33 bcde & $50.39 \mathrm{ab}$ & $57.44 \mathrm{cde}$ & $58.40 \mathrm{cdef}$ & $57.92 \mathrm{cdef}$ \\
\hline $\mathrm{T}_{9}$ & $\mathrm{~A}_{1} \mathrm{~B}_{1} \mathrm{C}_{0}$ & 42.88abcdef & 44.73abcd & 43.81abcdefg & $50.26 a b c$ & 51.27abcd & $50.77 \mathrm{ab}$ & $58.80 \mathrm{bcd}$ & $60.00 \mathrm{bcd}$ & $59.40 \mathrm{bcd}$ \\
\hline $\mathrm{T}_{10}$ & $\mathrm{~A}_{1} \mathrm{~B}_{1} \mathrm{C}_{1}$ & $47.48 \mathrm{ab}$ & $47.33 \mathrm{ab}$ & $47.41 \mathrm{a}$ & $53.80 \mathrm{a}$ & $53.43 \mathrm{abc}$ & $53.62 \mathrm{a}$ & $61.32 \mathrm{ab}$ & $62.30 \mathrm{ab}$ & $61.81 \mathrm{ab}$ \\
\hline $\mathrm{T}_{11}$ & $\mathrm{~A}_{1} \mathrm{~B}_{2} \mathrm{C}_{0}$ & $44.55 \mathrm{abc}$ & 44.9abcd & 44.73abcd & $51.30 \mathrm{ab}$ & 51.37abcd & $51.34 \mathrm{ab}$ & $59.05 \mathrm{bcd}$ & 60.30bcd & $59.68 \mathrm{bcd}$ \\
\hline $\mathrm{T}_{12}$ & $\mathrm{~A}_{1} \mathrm{~B}_{2} \mathrm{C}_{1}$ & $48.20 \mathrm{a}$ & $48.1 \mathrm{a}$ & $48.15 \mathrm{a}$ & $53.63 a$ & $54.8 \mathrm{a}$ & $54.22 \mathrm{a}$ & $63.10 \mathrm{a}$ & $63.60 \mathrm{a}$ & $63.35 a$ \\
\hline $\mathrm{T}_{13}$ & $\mathrm{~A}_{2} \mathrm{~B}_{0} \mathrm{C}_{0}$ & $40.15 \mathrm{cdefg}$ & 42.63abcd & $41.39 \mathrm{cdefgh}$ & $49.78 \mathrm{abc}$ & $50 \mathrm{cde}$ & $49.89 \mathrm{ab}$ & $56.82 \mathrm{def}$ & 57.37defg & 57.10defg \\
\hline $\mathrm{T}_{14}$ & $\mathrm{~A}_{2} \mathrm{~B}_{0} \mathrm{C}_{1}$ & $43.88 \mathrm{abc}$ & 44.47abcd & 44.18abcde & $50.38 \mathrm{abc}$ & 51.3abcd & $50.84 \mathrm{ab}$ & $58.52 \mathrm{bcd}$ & $59.80 \mathrm{bcde}$ & $59.16 \mathrm{bcde}$ \\
\hline $\mathrm{T}_{15}$ & $\mathrm{~A}_{2} \mathrm{~B}_{1} \mathrm{C}_{0}$ & 43.40abcde & 44abcd & 43.70abcdefg & $49.75 a b c$ & $50.3 \mathrm{bcde}$ & $50.03 \mathrm{ab}$ & $56.87 \mathrm{def}$ & $58.20 \mathrm{cdef}$ & $57.54 \mathrm{cdefg}$ \\
\hline $\mathrm{T}_{16}$ & $\mathrm{~A}_{2} \mathrm{~B}_{1} \mathrm{C}_{1}$ & $44.95 \mathrm{abc}$ & $45.3 \mathrm{abc}$ & $45.13 \mathrm{abc}$ & $50.75 a b c$ & 51.93abcd & $51.34 \mathrm{ab}$ & $59.87 \mathrm{bc}$ & $60.53 a b c$ & $60.20 b c$ \\
\hline $\mathrm{T}_{17}$ & $\mathrm{~A}_{2} \mathrm{~B}_{2} \mathrm{C}_{0}$ & 43.55abcde & 43.6abcd & 43.58abcdefg & $50.53 \mathrm{abc}$ & 50.53abcd & $50.53 \mathrm{ab}$ & 56.43def & 58.10cdef & 57.27defg \\
\hline $\mathrm{T}_{18}$ & $\mathrm{~A}_{2} \mathrm{~B}_{2} \mathrm{C}_{1}$ & $46.71 \mathrm{ab}$ & $46.4 \mathrm{abc}$ & $46.56 \mathrm{ab}$ & $53.46 \mathrm{a}$ & $54.47 \mathrm{ab}$ & $53.97 \mathrm{a}$ & $60.81 \mathrm{ab}$ & $62.07 \mathrm{ab}$ & $61.44 \mathrm{ab}$ \\
\hline \multicolumn{2}{|c|}{ S.Em. $( \pm)$} & 1.88 & 1.94 & 1.59 & 1.74 & 1.50 & 1.53 & 1.04 & 1.09 & 0.94 \\
\hline \multicolumn{2}{|c|}{ L.S.D. $(\mathrm{P} \leq 0.05)$} & 5.40 & 5.58 & 4.59 & 5.00 & 4.30 & 4.39 & 2.99 & 3.14 & 2.71 \\
\hline
\end{tabular}
MAP-Month after planting

**Means with the same letter are not significantly different 
Table.6 Effect of Nutrient management on D-leaf breadth $(\mathrm{cm})$

\begin{tabular}{|c|c|c|c|c|c|c|c|c|c|}
\hline \multirow[t]{2}{*}{ Treatments } & \multicolumn{3}{|c|}{3 MAP } & \multicolumn{3}{|c|}{6 MAP } & \multicolumn{3}{|c|}{9 MAP } \\
\hline & 2015 & 2016 & Mean & 2015 & 2016 & Mean & 2015 & 2016 & Mean \\
\hline $\mathrm{A}_{0}$ & $3.08 \mathrm{c}$ & $3.18 \mathrm{~b}$ & $3.13 \mathrm{c}$ & $3.18 \mathrm{c}$ & $3.24 b$ & $3.21 \mathrm{c}$ & $3.33 \mathrm{~b}$ & $3.41 \mathrm{~b}$ & $3.37 \mathrm{~b}$ \\
\hline $\mathrm{A}_{1}$ & $3.76 \mathrm{a}$ & $4.02 \mathrm{a}$ & $3.89 a$ & $3.92 \mathrm{a}$ & $4.21 \mathrm{a}$ & $4.06 \mathrm{a}$ & $4.12 \mathrm{a}$ & $4.29 a$ & $4.21 \mathrm{a}$ \\
\hline $\mathrm{A}_{2}$ & $3.35 \mathrm{a}$ & $3.89 a$ & $3.62 b$ & $3.49 \mathrm{~b}$ & $4.06 \mathrm{a}$ & $3.78 \mathrm{~b}$ & $3.73 \mathrm{ab}$ & $4.16 \mathrm{a}$ & $3.95 \mathrm{a}$ \\
\hline S.Em. ( $( \pm)$ & 0.07 & 0.10 & 0.07 & 0.07 & 0.13 & 0.08 & 0.14 & 0.15 & 0.15 \\
\hline L.S.D(P $\leq 0.05)$ & 0.19 & 0.28 & 0.21 & 0.19 & 0.36 & 0.24 & 0.41 & 0.44 & 0.42 \\
\hline $\mathrm{B}_{0}$ & $3.38 \mathrm{a}$ & $3.58 \mathrm{a}$ & $3.48 \mathrm{a}$ & $3.42 \mathrm{~b}$ & $3.74 \mathrm{a}$ & $3.58 \mathrm{a}$ & $3.62 \mathrm{a}$ & $3.75 \mathrm{a}$ & $3.69 \mathrm{a}$ \\
\hline $\mathrm{B}_{1}$ & $3.38 \mathrm{a}$ & $3.72 \mathrm{a}$ & $3.55 \mathrm{a}$ & $3.52 \mathrm{ab}$ & $3.88 \mathrm{a}$ & $3.70 \mathrm{a}$ & $3.73 \mathrm{a}$ & $4.03 a$ & $3.88 \mathrm{a}$ \\
\hline $\mathrm{B}_{2}$ & $3.43 \mathrm{a}$ & $3.78 \mathrm{a}$ & $3.61 \mathrm{a}$ & $3.65 \mathrm{a}$ & $3.90 \mathrm{a}$ & $3.78 \mathrm{a}$ & $3.84 \mathrm{a}$ & $4.08 \mathrm{a}$ & $3.96 \mathrm{a}$ \\
\hline S.Em. $( \pm)$ & 0.07 & 0.10 & 0.07 & 0.07 & 0.13 & 0.08 & 0.14 & 0.15 & 0.15 \\
\hline L.S.D(P $\leq 0.05)$ & NS & NS & NS & 0.19 & NS & NS & NS & NS & NS \\
\hline $\mathrm{C}_{0}$ & $3.28 \mathrm{~b}$ & $3.58 \mathrm{a}$ & $3.43 \mathrm{~b}$ & $3.43 \mathrm{~b}$ & $3.75 b$ & $3.59 \mathrm{a}$ & $3.60 \mathrm{a}$ & $3.81 \mathrm{a}$ & $3.71 \mathrm{a}$ \\
\hline $\mathrm{C}_{1}$ & $3.51 \mathrm{a}$ & $3.81 \mathrm{a}$ & $3.66 \mathrm{a}$ & $3.63 \mathrm{a}$ & $3.93 \mathrm{a}$ & $3.78 \mathrm{a}$ & $3.85 \mathrm{a}$ & $4.09 a$ & $3.98 \mathrm{a}$ \\
\hline S.Em. $( \pm)$ & 0.05 & 0.08 & 0.06 & 0.05 & 0.10 & 0.07 & 0.12 & 0.12 & 0.12 \\
\hline L.S.D(P $\leq 0.05)$ & 0.15 & NS & 0.17 & 0.15 & 0.29 & NS & NS & NS & NS \\
\hline
\end{tabular}

\begin{tabular}{|c|c|c|c|c|c|c|c|c|c|c|}
\hline \multirow{2}{*}{\multicolumn{2}{|c|}{$\begin{array}{l}\text { Treatments/ } \\
\text { Combination }\end{array}$}} & \multicolumn{3}{|c|}{3 MAP } & \multicolumn{3}{|c|}{6 MAP } & \multicolumn{3}{|c|}{ 9 MAP } \\
\hline & & \multirow{2}{*}{\begin{tabular}{|l}
2015 \\
$3.03 \mathrm{~g}$
\end{tabular}} & \multirow{2}{*}{$\begin{array}{r}\mathbf{2 0 1 6} \\
3.03 \mathrm{f}\end{array}$} & \multirow{2}{*}{$\begin{array}{l}\text { Mean } \\
3.03 \mathrm{e}^{-}\end{array}$} & \multirow{2}{*}{2015} & 2016 & \multirow{2}{*}{ Mean } & \multirow{2}{*}{$\frac{2015}{0 c}$} & 2016 & \multirow{2}{*}{$\begin{array}{r}\text { Mean } \\
3.27 \mathrm{c}\end{array}$} \\
\hline $\mathrm{T}_{1}$ & $\mathrm{~A}_{0} \mathrm{~B}_{0} \mathrm{C}_{0}$ & & & & & $3.08 \mathrm{e}$ & & & $3.23 \mathrm{~d}$ & \\
\hline$T_{2}$ & $\mathrm{~A}_{0} \mathrm{~B}_{0} \mathrm{C}_{1}$ & $3.11 \mathrm{efg}$ & $3.22 \mathrm{cdef}$ & $3.17 \mathrm{de}$ & $3.26 \mathrm{de}$ & $3.24 \mathrm{cde}$ & $3.25 \mathrm{efg}$ & $3.40 \mathrm{bc}$ & $3.41 \mathrm{~cd}$ & $3.41 \mathrm{c}$ \\
\hline $\mathrm{T}_{3}$ & $\mathrm{~A}_{0} \mathrm{~B}_{1} \mathrm{C}_{0}$ & $3.14 \mathrm{efg}$ & $3.11 \mathrm{ef}$ & $3.13 \mathrm{e}$ & $3.23 \mathrm{de}$ & $3.2 \mathrm{de}$ & $3.22 \mathrm{fg}$ & $3.28 \mathrm{c}$ & $3.33 \mathrm{~cd}$ & $3.31 \mathrm{c}$ \\
\hline $\mathrm{T}_{4}$ & $\mathrm{~A}_{0} \mathrm{~B}_{1} \mathrm{C}_{1}$ & $3.02 \mathrm{~g}$ & $3.12 \mathrm{ef}$ & $3.07 \mathrm{e}$ & $3.10 \mathrm{e}$ & 3.34bcde & $3.22 \mathrm{fg}$ & $3.25 \mathrm{c}$ & $3.49 \mathrm{bcd}$ & $3.37 \mathrm{c}$ \\
\hline $\mathrm{T}_{5}$ & $\mathrm{~A}_{0} \mathrm{~B}_{2} \mathrm{C}_{0}$ & $3.12 \mathrm{efg}$ & 3.18def & $3.15 \mathrm{e}$ & $3.14 \mathrm{e}$ & $3.21 \mathrm{de}$ & $3.18 \mathrm{fg}$ & $3.30 \mathrm{c}$ & $3.42 \mathrm{~cd}$ & $3.36 \mathrm{c}$ \\
\hline $\mathrm{T}_{6}$ & $\mathrm{~A}_{0} \mathrm{~B}_{2} \mathrm{C}_{1}$ & $3.07 \mathrm{fg}$ & $3.27 \mathrm{bcdef}$ & $3.17 \mathrm{cde}$ & $3.23 \mathrm{de}$ & 3.39bcde & 3.31defg & $3.45 \mathrm{abc}$ & $3.55 \mathrm{bcd}$ & $3.50 \mathrm{bc}$ \\
\hline $\mathrm{T}_{7}$ & $\mathrm{~A}_{1} \mathrm{~B}_{0} \mathrm{C}_{0}$ & 3.51 bcdef & $3.80 \mathrm{abcd}$ & $3.66 \mathrm{bcd}$ & $3.69 \mathrm{bcd}$ & $4.02 \mathrm{abcd}$ & $3.86 \mathrm{abcd}$ & $3.84 \mathrm{abc}$ & 3.73abcd & $3.79 \mathrm{abc}$ \\
\hline $\mathrm{T}_{8}$ & $\mathrm{~A}_{1} \mathrm{~B}_{0} \mathrm{C}_{1}$ & 3.64abcd & 3.84abcd & $3.74 \mathrm{abc}$ & $3.89 \mathrm{abc}$ & 4.06abcd & $3.98 \mathrm{abc}$ & $3.99 \mathrm{abc}$ & 4.2abcd & 4.10abc \\
\hline $\mathrm{T}_{9}$ & $\mathrm{~A}_{1} \mathrm{~B}_{1} \mathrm{C}_{0}$ & 3.53bcde & $3.9 \mathrm{abc}$ & $3.72 \mathrm{abc}$ & $3.75 a b c$ & $4.12 \mathrm{abc}$ & $3.94 \mathrm{abc}$ & $4.05 \mathrm{abc}$ & 4.26abcd & $4.16 a b c$ \\
\hline $\mathrm{T}_{10}$ & $\mathrm{~A}_{1} \mathrm{~B}_{1} \mathrm{C}_{1}$ & $4.09 \mathrm{a}$ & $4.32 \mathrm{a}$ & $4.21 \mathrm{a}$ & $4.13 \mathrm{ab}$ & $4.38 \mathrm{a}$ & $4.26 \mathrm{ab}$ & $4.35 \mathrm{ab}$ & $4.56 \mathrm{ab}$ & $4.46 \mathrm{ab}$ \\
\hline $\mathrm{T}_{11}$ & $\mathrm{~A}_{1} \mathrm{~B}_{2} \mathrm{C}_{0}$ & $3.75 a b c$ & 3.91abc & $3.83 \mathrm{ab}$ & $3.86 a b c$ & $4.13 \mathrm{ab}$ & $4.00 \mathrm{abc}$ & $4.07 \mathrm{abc}$ & 4.28abcd & $4.18 \mathrm{abc}$ \\
\hline $\mathrm{T}_{12}$ & $\mathrm{~A}_{1} \mathrm{~B}_{2} \mathrm{C}_{1}$ & $4.02 \mathrm{a}$ & $4.32 \mathrm{a}$ & $4.17 \mathrm{a}$ & $4.19 a$ & $4.54 \mathrm{a}$ & $4.37 \mathrm{a}$ & $4.44 a$ & $4.7 \mathrm{a}$ & $4.57 \mathrm{a}$ \\
\hline $\mathrm{T}_{13}$ & $\mathrm{~A}_{2} \mathrm{~B}_{0} \mathrm{C}_{0}$ & $3.05 \mathrm{fg}$ & 3.75abcde & 3.40bcde & $3.11 \mathrm{e}$ & 3.97abcd & 3.54cdefg & $3.34 \mathrm{c}$ & 3.71abcd & $3.53 \mathrm{bc}$ \\
\hline $\mathrm{T}_{14}$ & $\mathrm{~A}_{2} \mathrm{~B}_{0} \mathrm{C}_{1}$ & $3.25 \mathrm{ab}$ & 3.82abcd & $3.54 \mathrm{ab}$ & $3.45 \mathrm{cde}$ & 4.04abcd & 3.75bcdef & $3.84 \mathrm{abc}$ & 4.21abcd & $4.03 \mathrm{abc}$ \\
\hline $\mathrm{T}_{15}$ & $\mathrm{~A}_{2} \mathrm{~B}_{1} \mathrm{C}_{0}$ & 3.16efg & 3.82abcde & $3.49 \mathrm{bcde}$ & 3.29de & 4.04abcd & 3.67cdefg & $3.50 \mathrm{abc}$ & 4.19abcd & $3.85 \mathrm{abc}$ \\
\hline $\mathrm{T}_{16}$ & $\mathrm{~A}_{2} \mathrm{~B}_{1} \mathrm{C}_{1}$ & $3.32 \mathrm{cdefg}$ & $4.09 \mathrm{ab}$ & $3.71 \mathrm{abc}$ & $3.64 \mathrm{~cd}$ & $4.18 \mathrm{ab}$ & $3.91 \mathrm{abc}$ & $3.96 \mathrm{abc}$ & $4.33 \mathrm{abc}$ & $4.15 \mathrm{abc}$ \\
\hline $\mathrm{T}_{17}$ & $\mathrm{~A}_{2} \mathrm{~B}_{2} \mathrm{C}_{0}$ & 3.24defg & 3.74abcde & $3.49 \mathrm{bcde}$ & $3.64 \mathrm{~cd}$ & 3.96abcde & 3.80abcde & $3.75 a b c$ & 4.12abcd & $3.94 a b c$ \\
\hline $\mathrm{T}_{18}$ & $\mathrm{~A}_{2} \mathrm{~B}_{2} \mathrm{C}_{1}$ & $3.37 \mathrm{cdefg}$ & $4.14 \mathrm{a}$ & $3.76 \mathrm{ab}$ & $3.82 \mathrm{abc}$ & $4.19 \mathrm{ab}$ & $4.01 \mathrm{abc}$ & $4.01 \mathrm{abc}$ & $4.38 \mathrm{abc}$ & $4.20 \mathrm{abc}$ \\
\hline \multicolumn{2}{|c|}{ S.Em. $( \pm)$} & 0.16 & 0.24 & 0.18 & 0.16 & 0.31 & 0.20 & 0.35 & 0.37 & 0.36 \\
\hline \multicolumn{2}{|c|}{ L.S.D. $(\mathrm{P} \leq 0.05)$} & 0.46 & 0.70 & 0.50 & 0.46 & 0.88 & 0.58 & 0.01 & 1.07 & 1.03 \\
\hline
\end{tabular}

MAP-Month after planting

**Means with the same letter are not significantly different 
Table.6 Effect of Nutrient management on D-leaf breadth $(\mathrm{cm})($ contd....)

\begin{tabular}{|c|c|c|c|c|c|c|c|c|c|}
\hline \multirow[t]{2}{*}{ Treatments } & \multicolumn{3}{|c|}{12 MAP } & \multicolumn{3}{|c|}{15 MAP } & \multicolumn{3}{|c|}{18 MAP } \\
\hline & 2015 & 2016 & Mean & 2015 & 2016 & Mean & 2015 & 2016 & Mean \\
\hline $\mathrm{A}_{0}$ & $3.54 \mathrm{~b}$ & $3.81 \mathrm{~b}$ & $3.68 \mathrm{~b}$ & $3.76 \mathrm{~b}$ & $3.84 \mathrm{~b}$ & $3.80 \mathrm{~b}$ & $3.97 \mathrm{~b}$ & $4.06 \mathrm{~b}$ & $4.02 \mathrm{~b}$ \\
\hline $\mathrm{A}_{1}$ & $4.50 \mathrm{a}$ & $4.54 \mathrm{a}$ & $4.52 \mathrm{a}$ & $4.54 \mathrm{a}$ & $4.63 \mathrm{a}$ & $4.58 \mathrm{a}$ & $4.73 a$ & $4.85 \mathrm{a}$ & $4.79 a$ \\
\hline $\mathrm{A}_{2}$ & $4.32 \mathrm{a}$ & $4.34 \mathrm{a}$ & $4.32 \mathrm{a}$ & $4.38 \mathrm{a}$ & $4.52 \mathrm{a}$ & $4.45 \mathrm{a}$ & $4.58 \mathrm{a}$ & $4.72 \mathrm{a}$ & $4.65 \mathrm{a}$ \\
\hline S.Em. ( \pm$)$ & 0.09 & 0.11 & 0.09 & 0.09 & 0.09 & 0.09 & 0.09 & 0.10 & 0.10 \\
\hline L.S.D(P $\leq 0.05)$ & 0.25 & 0.32 & 0.27 & 0.27 & 0.27 & 0.27 & 0.27 & 0.29 & 0.28 \\
\hline $\mathrm{B}_{0}$ & $3.98 \mathrm{~b}$ & $4.07 \mathrm{~b}$ & $4.02 \mathrm{~b}$ & $4.12 \mathrm{a}$ & $4.32 \mathrm{a}$ & $4.18 \mathrm{a}$ & $4.28 \mathrm{~b}$ & $4.41 \mathrm{a}$ & $4.35 \mathrm{a}$ \\
\hline $\mathrm{B}_{1}$ & $4.13 \mathrm{ab}$ & $4.19 \mathrm{~b}$ & $4.16 \mathrm{ab}$ & $4.19 \mathrm{a}$ & $4.32 \mathrm{a}$ & $4.26 \mathrm{a}$ & $4.43 \mathrm{ab}$ & $4.55 \mathrm{a}$ & $4.49 \mathrm{a}$ \\
\hline $\mathrm{B}_{2}$ & $4.26 \mathrm{a}$ & $4.42 \mathrm{a}$ & $4.33 \mathrm{a}$ & $4.35 \mathrm{a}$ & $4.45 \mathrm{a}$ & $4.40 \mathrm{a}$ & $4.56 \mathrm{a}$ & $4.67 \mathrm{a}$ & $4.12 \mathrm{a}$ \\
\hline S.Em. ( \pm$)$ & 0.09 & 0.11 & 0.09 & 0.09 & 0.09 & 0.09 & 0.09 & 0.10 & 0.10 \\
\hline L.S.D(P $\leq 0.05)$ & 0.25 & 0.32 & 0.27 & NS & NS & NS & 0.27 & NS & NS \\
\hline $\mathrm{C}_{0}$ & $3.98 \mathrm{~b}$ & $4.02 \mathrm{~b}$ & $4.00 \mathrm{~b}$ & $4.09 \mathrm{~b}$ & $4.22 \mathrm{a}$ & $4.16 \mathrm{~b}$ & $4.28 \mathrm{~b}$ & $4.40 \mathrm{~b}$ & $4.34 \mathrm{~b}$ \\
\hline $\mathrm{C}_{1}$ & $4.27 \mathrm{a}$ & $4.44 a$ & $4.35 \mathrm{a}$ & $4.39 a$ & $4.44 a$ & $4.40 \mathrm{a}$ & $4.57 \mathrm{a}$ & $4.69 a$ & $4.63 a$ \\
\hline S.Em. ( \pm$)$ & 0.07 & 0.09 & 0.08 & 0.08 & 0.08 & 0.08 & 0.08 & 0.08 & 0.08 \\
\hline L.S.D(P $\leq 0.05)$ & 0.20 & 0.26 & 0.22 & 0.22 & NS & 0.22 & 0.22 & 0.24 & 0.23 \\
\hline
\end{tabular}

\begin{tabular}{|c|c|c|c|c|c|c|c|c|c|c|}
\hline \multirow{2}{*}{\multicolumn{2}{|c|}{$\begin{array}{l}\text { Treatments/ } \\
\text { Combination }\end{array}$}} & \multicolumn{3}{|c|}{12 MAP } & \multicolumn{3}{|c|}{15 MAP } & \multicolumn{3}{|c|}{18 MAP } \\
\hline & & \multirow{2}{*}{$\begin{array}{l}\mathbf{2 0 1 5} \\
3.40 \mathrm{f}\end{array}$} & \multirow{2}{*}{$\begin{array}{l}\mathbf{2 0 1 6} \\
3.44 \mathrm{f} \\
\end{array}$} & \multirow{2}{*}{\begin{tabular}{|l} 
Mean \\
$3.42 \mathrm{~g}$ \\
\end{tabular}} & \multirow{2}{*}{$3.46 \mathrm{e}^{\mathbf{2 0 1 5}}$} & 2016 & \multirow{2}{*}{$\begin{array}{l}\text { Mean } \\
3.51 \mathrm{e}^{\text {Mean }}\end{array}$} & \multirow{2}{*}{\begin{tabular}{|l}
2015 \\
$3.68 \mathrm{f}$
\end{tabular}} & 2016 & \multirow{2}{*}{$\begin{array}{l}\text { Mean } \\
3.71 \mathrm{e}^{\text {Men }}\end{array}$} \\
\hline $\mathrm{T}_{1}$ & $\mathrm{~A}_{0} \mathrm{~B}_{0} \mathrm{C}_{0}$ & & & & & $3.55 \mathrm{f}$ & & & $3.75 \mathrm{e}$ & \\
\hline $\mathrm{T}_{2}$ & $\mathrm{~A}_{0} \mathrm{~B}_{0} \mathrm{C}_{1}$ & $3.58 \mathrm{ef}$ & $3.88 \mathrm{cdef}$ & $3.73 \mathrm{efg}$ & $3.82 \mathrm{cde}$ & 3.93def & $3.88 \mathrm{cde}$ & $4.05 \mathrm{cdef}$ & $4.19 \mathrm{cde}$ & $4.12 \mathrm{cde}$ \\
\hline $\mathrm{T}_{3}$ & $\mathrm{~A}_{0} \mathrm{~B}_{1} \mathrm{C}_{0}$ & $3.51 \mathrm{ef}$ & $3.53 \mathrm{f}$ & $3.52 \mathrm{fg}$ & $3.55 \mathrm{e}$ & $3.63 \mathrm{f}$ & $3.59 \mathrm{e}$ & $3.81 \mathrm{ef}$ & $3.84 \mathrm{e}$ & $3.83 \mathrm{e}$ \\
\hline $\mathrm{T}_{4}$ & $\mathrm{~A}_{0} \mathrm{~B}_{1} \mathrm{C}_{1}$ & $3.52 \mathrm{ef}$ & 3.85def & $3.69 \mathrm{efgt}$ & $3.91 \mathrm{cde}$ & $3.94 \mathrm{cdef}$ & $3.93 \mathrm{cde}$ & $4.04 \mathrm{cdef}$ & $4.16 \mathrm{cde}$ & 4.10cde \\
\hline $\mathrm{T}_{5}$ & $\mathrm{~A}_{0} \mathrm{~B}_{2} \mathrm{C}_{0}$ & $3.56 \mathrm{ef}$ & $3.71 \mathrm{ef}$ & $3.64 \mathrm{fg}$ & $3.72 \mathrm{de}$ & $3.83 \mathrm{ef}$ & $3.78 \mathrm{de}$ & $3.96 \mathrm{def}$ & $4.02 \mathrm{de}$ & 3.99de \\
\hline $\mathrm{T}_{6}$ & $\mathrm{~A}_{0} \mathrm{~B}_{2} \mathrm{C}_{1}$ & 3.70ef & 4.44abcde & 4.07cdefg & 4.07bcde & 4.19bcdef & $4.13 \mathrm{bcde}$ & 4.28abcdef & $4.42 \mathrm{bcde}$ & $4.35 \mathrm{bcde}$ \\
\hline $\mathrm{T}_{7}$ & $\mathrm{~A}_{1} \mathrm{~B}_{0} \mathrm{C}_{0}$ & 4.11bcde & 4.14abcdef & $4.13 \mathrm{bcde}$ & 4.34abcd & 4.48abcde & 4.41abcd & $4.47 \mathrm{bcd}$ & 4.67abcd & 4.57abcd \\
\hline $\mathrm{T}_{8}$ & $\mathrm{~A}_{1} \mathrm{~B}_{0} \mathrm{C}_{1}$ & 4.40abcd & 4.42abcde & 4.41abcd & $4.45 \mathrm{abc}$ & 4.42abcde & 4.44abc & 4.50abcd & 4.64abcd & 4.57abcd \\
\hline $\mathrm{T}_{9}$ & $\mathrm{~A}_{1} \mathrm{~B}_{1} \mathrm{C}_{0}$ & 4.50abcd & 4.48abcde & 4.49abcd & $4.41 \mathrm{abc}$ & $4.60 \mathrm{abc}$ & $4.51 \mathrm{abc}$ & $4.62 \mathrm{abc}$ & 4.79abc & $4.70 \mathrm{abc}$ \\
\hline $\mathrm{T}_{10}$ & $\mathrm{~A}_{1} \mathrm{~B}_{1} \mathrm{C}_{1}$ & $4.68 \mathrm{ab}$ & $4.78 \mathrm{ab}$ & $4.73 \mathrm{ab}$ & $4.64 \mathrm{ab}$ & $4.76 \mathrm{ab}$ & $4.70 \mathrm{ab}$ & $4.90 \mathrm{ab}$ & $4.97 \mathrm{ab}$ & $4.93 \mathrm{ab}$ \\
\hline $\mathrm{T}_{11}$ & $\mathrm{~A}_{1} \mathrm{~B}_{2} \mathrm{C}_{0}$ & 4.44abcd & 4.51abcd & 4.48abcd & $4.41 \mathrm{abc}$ & $4.62 \mathrm{ab}$ & $4.52 \mathrm{abc}$ & $4.72 \mathrm{ab}$ & $4.82 \mathrm{abc}$ & $4.77 \mathrm{abc}$ \\
\hline $\mathrm{T}_{12}$ & $\mathrm{~A}_{1} \mathrm{~B}_{2} \mathrm{C}_{1}$ & $4.85 \mathrm{a}$ & $4.88 \mathrm{a}$ & $4.87 \mathrm{a}$ & $4.97 \mathrm{a}$ & $4.9 \mathrm{a}$ & $4.94 \mathrm{a}$ & $5.15 \mathrm{a}$ & $5.18 \mathrm{a}$ & $5.17 \mathrm{a}$ \\
\hline $\mathrm{T}_{13}$ & $\mathrm{~A}_{2} \mathrm{~B}_{0} \mathrm{C}_{0}$ & 3.97cdef & 4.06bcdef & 4.02cdefg & $4.31 \mathrm{bcd}$ & 4.39abcde & 4.35abcd & $4.31 \mathrm{bcdef}$ & $4.37 \mathrm{bcde}$ & 4.34bcde \\
\hline $\mathrm{T}_{14}$ & $\mathrm{~A}_{2} \mathrm{~B}_{0} \mathrm{C}_{1}$ & 4.41abcd & 4.48abcde & 4.45abcd & 4.37abcd & 4.57abcd & $4.47 \mathrm{abc}$ & 4.69abc & $4.82 \mathrm{abc}$ & 4.76abc \\
\hline $\mathrm{T}_{15}$ & $\mathrm{~A}_{2} \mathrm{~B}_{1} \mathrm{C}_{0}$ & 3.97def & $3.96 \mathrm{cdef}$ & 3.97defg & $4.31 \mathrm{bcd}$ & 4.48abcde & 4.40abcd & 4.49abcd & 4.69abcd & 4.59abcd \\
\hline $\mathrm{T}_{16}$ & $\mathrm{~A}_{2} \mathrm{~B}_{1} \mathrm{C}_{1}$ & $4.58 \mathrm{abc}$ & 4.55abcd & 4.57abcd & 4.35abcd & 4.54abcd & $4.45 \mathrm{abc}$ & $4.71 \mathrm{ab}$ & $4.85 \mathrm{abc}$ & $4.78 \mathrm{abc}$ \\
\hline $\mathrm{T}_{17}$ & $\mathrm{~A}_{2} \mathrm{~B}_{2} \mathrm{C}_{0}$ & 4.34abcd & 4.32abcde & 4.33abcde & 4.32abcd & 4.44abcde & 4.38abcd & 4.46bcde & 4.63abcd & 4.55abcd \\
\hline $\mathrm{T}_{18}$ & $\mathrm{~A}_{2} \mathrm{~B}_{2} \mathrm{C}_{1}$ & $4.66 \mathrm{ab}$ & $4.64 a b c$ & $4.65 \mathrm{abc}$ & $4.59 \mathrm{ab}$ & $4.70 \mathrm{ab}$ & $4.65 \mathrm{ab}$ & $4.81 \mathrm{ab}$ & $4.94 \mathrm{ab}$ & $4.87 \mathrm{ab}$ \\
\hline \multicolumn{2}{|c|}{ S.Em. $( \pm)$} & 0.21 & 0.27 & 0.23 & 0.23 & 0.23 & 0.23 & 0.23 & 0.25 & 0.24 \\
\hline \multicolumn{2}{|c|}{ L.S.D. $(\mathrm{P} \leq 0.05)$} & 0.61 & 0.78 & 0.65 & 0.65 & 0.67 & 0.65 & 0.66 & 0.72 & 0.68 \\
\hline
\end{tabular}


Table.7 Effect of Nutrient management on D-leaf area $\left(\mathrm{cm}^{2}\right)$

\begin{tabular}{|c|c|c|c|c|c|c|c|c|c|}
\hline \multirow[t]{2}{*}{ Treatments } & \multicolumn{3}{|c|}{3 MAP } & \multicolumn{3}{|c|}{6 MAP } & \multicolumn{3}{|c|}{9 MAP } \\
\hline & 2015 & 2016 & Mean & 2015 & 2016 & Mean & 2015 & 2016 & Mean \\
\hline$\overline{\mathrm{A}_{0}}$ & $61.70 c$ & $62.72 b$ & $62.21 \mathrm{c}$ & $78.31 \mathrm{c}$ & $81.84 b$ & $80.07 \mathrm{c}$ & $99.19 \mathrm{c}$ & $111.79 b$ & $105.49 b$ \\
\hline $\mathrm{A}_{1}$ & $84.73 a$ & $91.62 \mathrm{a}$ & $88.17 \mathrm{a}$ & $109.15 \mathrm{a}$ & $118.24 \mathrm{a}$ & $113.69 \mathrm{a}$ & $143.12 \mathrm{a}$ & $154.65 \mathrm{a}$ & $148.90 \mathrm{a}$ \\
\hline$A_{2}$ & $75.25 \mathrm{~b}$ & $87.30 \mathrm{a}$ & $81.27 \mathrm{~b}$ & $95.43 b$ & $112.71 \mathrm{a}$ & $104.07 \mathrm{~b}$ & $127.19 \mathrm{~b}$ & $147.52 \mathrm{a}$ & $137.36 \mathrm{a}$ \\
\hline S.Em. ( $( \pm)$ & 1.76 & 2.63 & 1.99 & 2.21 & 3.79 & 2.66 & 4.74 & 5.35 & 4.97 \\
\hline L.S.D(P $\leq 0.05)$ & 5.07 & 7.55 & 5.72 & 6.35 & 10.89 & 7.66 & 13.62 & 15.37 & 14.28 \\
\hline $\mathrm{B}_{0}$ & $72.61 \mathrm{a}$ & $76.28 \mathrm{a}$ & $74.44 a$ & $90.77 \mathrm{~b}$ & $99.48 \mathrm{a}$ & $95.13 \mathrm{a}$ & $116.99 \mathrm{a}$ & $128.16 b$ & $122.57 \mathrm{a}$ \\
\hline $\mathrm{B}_{1}$ & $72.25 \mathrm{a}$ & $82.05 \mathrm{a}$ & $77.15 \mathrm{a}$ & $93.87 \mathrm{~b}$ & $106.05 \mathrm{a}$ & $99.96 \mathrm{a}$ & $124.12 \mathrm{a}$ & $141.60 \mathrm{~b}$ & $132.86 a$ \\
\hline $\mathrm{B}_{2}$ & $76.82 a$ & $83.31 \mathrm{a}$ & $80.06 a$ & $98.24 \mathrm{a}$ & $107.25 \mathrm{a}$ & $102.74 a$ & $128.42 \mathrm{a}$ & $144.20 \mathrm{a}$ & $136.31 \mathrm{a}$ \\
\hline S.Em. ( \pm$)$ & 1.76 & 2.63 & 1.99 & 2.21 & 3.79 & 2.66 & 4.74 & 5.35 & 4.97 \\
\hline L.S.D(P $\leq 0.05)$ & NS & NS & NS & 6.35 & NS & NS & NS & 15.37 & NS \\
\hline $\mathrm{C}_{0}$ & $69.81 \mathrm{~b}$ & $76.67 \mathrm{~b}$ & $73.24 \mathrm{~b}$ & $90.03 b$ & $100.54 \mathrm{a}$ & $95.29 \mathrm{~b}$ & $116.45 b$ & $130.59 \mathrm{~b}$ & $123.52 b$ \\
\hline $\mathrm{C}_{1}$ & $77.97 \mathrm{a}$ & $84.42 \mathrm{a}$ & $81.20 \mathrm{a}$ & $98.55 \mathrm{a}$ & $107.98 \mathrm{a}$ & $103.27 \mathrm{a}$ & $129.90 \mathrm{a}$ & $145.38 \mathrm{a}$ & $137.64 a$ \\
\hline S.Em. ( $( \pm)$ & 1.44 & 2.14 & 1.63 & 1.80 & 3.09 & 2.17 & 3.87 & 4.37 & 4.06 \\
\hline L.S.D(P $\leq 0.05)$ & 4.14 & 6.16 & 4.67 & 5.18 & NS & 6.25 & 11.12 & 12.55 & 11.06 \\
\hline
\end{tabular}

\begin{tabular}{|c|c|c|c|c|c|c|c|c|c|c|}
\hline \multirow{2}{*}{\multicolumn{2}{|c|}{$\begin{array}{l}\text { Treatments/ } \\
\text { Combination }\end{array}$}} & \multicolumn{3}{|c|}{3 МАР } & \multicolumn{3}{|c|}{6 МАP } & \multicolumn{3}{|c|}{9 MAP } \\
\hline & & \multirow{2}{*}{$\begin{array}{r}\mathbf{2 0 1 5} \\
52.85 \mathrm{f}\end{array}$} & \multirow{2}{*}{$\begin{array}{r}\mathbf{2 0 1 6} \\
54.39 \mathrm{f}\end{array}$} & \multirow{2}{*}{$\begin{array}{l}\text { Mean } \\
53.62 \mathrm{e}\end{array}$} & \multirow{2}{*}{$\begin{array}{r}\mathbf{2 0 1 5} \\
71.97 \mathrm{i}\end{array}$} & \multirow{2}{*}{$\begin{array}{c}\mathbf{2 0 1 6} \\
72.92 \mathrm{f}\end{array}$} & \multirow{2}{*}{$\begin{array}{r}\text { Mean } \\
72.45 \mathrm{~g}\end{array}$} & \multirow{2}{*}{$\begin{array}{l}\mathbf{2 0 1 5} \\
94.53 \mathrm{~g}\end{array}$} & \multirow{2}{*}{2016} & \multirow{2}{*}{$\begin{array}{c}\text { Mean } \\
96.37 \mathrm{~g} \\
\end{array}$} \\
\hline $\mathrm{T}_{1}$ & $\mathrm{~A}_{0} \mathrm{~B}_{0} \mathrm{C}_{0}$ & & & & & & & & & \\
\hline $\mathrm{T}_{2}$ & $\mathrm{~A}_{0} \mathrm{~B}_{0} \mathrm{C}_{1}$ & $67.34 \mathrm{cde}$ & $65.07 \mathrm{ef}$ & $66.21 \mathrm{de}$ & 80.93 ghi & 82.73 def & $81.83 \mathrm{efg}$ & $102.51 \mathrm{efg}$ & 114.90 efgh & $108.71 \mathrm{efg}$ \\
\hline $\mathrm{T}_{3}$ & $\mathrm{~A}_{0} \mathrm{~B}_{1} \mathrm{C}_{0}$ & $57.58 \mathrm{ef}$ & $61.03 \mathrm{f}$ & $59.31 \mathrm{e}$ & 81.65 fghi & $81.05 \mathrm{f}$ & $81.35 \mathrm{efg}$ & $96.06 \mathrm{fg}$ & $107.27 \mathrm{gh}$ & $101.67 \mathrm{fg}$ \\
\hline $\mathrm{T}_{4}$ & $\mathrm{~A}_{0} \mathrm{~B}_{1} \mathrm{C}_{1}$ & $59.47 \mathrm{ef}$ & $63.62 \mathrm{f}$ & $61.55 \mathrm{e}$ & $79.08 \mathrm{hi}$ & 84.23 cdef & 81.66 efg & $98.16 \mathrm{fg}$ & 116.55 efgh & $107.36 \mathrm{efg}$ \\
\hline $\mathrm{T}_{5}$ & $\mathrm{~A}_{0} \mathrm{~B}_{2} \mathrm{C}_{0}$ & $68.73 \mathrm{cde}$ & $63.74 f$ & $66.24 \mathrm{de}$ & $78.22 \mathrm{hi}$ & $81.55 \mathrm{ef}$ & $79.89 \mathrm{fg}$ & $97.23 \mathrm{fg}$ & $112.97 \mathrm{fgh}$ & $105.10 \mathrm{fg}$ \\
\hline $\mathrm{T}_{6}$ & $\mathrm{~A}_{0} \mathrm{~B}_{2} \mathrm{C}_{1}$ & $64.23 \mathrm{def}$ & $68.45 \mathrm{def}$ & $66.34 \mathrm{de}$ & $77.98 \mathrm{hi}$ & 88.53 bcdef & 83.26 defg & 106.67 defg & 120.84 defgh & 113.76 defg \\
\hline $\mathrm{T}_{7}$ & $\mathrm{~A}_{1} \mathrm{~B}_{0} \mathrm{C}_{0}$ & $75.12 \mathrm{bcd}$ & $84.95 \mathrm{abcd}$ & $80.04 \mathrm{~cd}$ & 102.16 bcde & $109.83 \mathrm{abc}$ & $106.00 \mathrm{bc}$ & 128.71 abcdef & 130.12 bcdefgh & 129.42 bcdefg \\
\hline $\mathrm{T}_{8}$ & $\mathrm{~A}_{1} \mathrm{~B}_{0} \mathrm{C}_{1}$ & $85.40 \mathrm{ab}$ & $85.6 \mathrm{abcd}$ & $85.50 \mathrm{abc}$ & $108.96 \mathrm{abc}$ & $111.85 \mathrm{ab}$ & $110.41 \mathrm{abc}$ & 135.82 abcde & 148.5 abcdef & 142.16 abcde \\
\hline $\mathrm{T}_{9}$ & $\mathrm{~A}_{1} \mathrm{~B}_{1} \mathrm{C}_{0}$ & $76.38 \mathrm{bcd}$ & $88.94 a b c$ & $82.66 \mathrm{bc}$ & 102.05 bcde & $116.03 \mathrm{a}$ & $109.04 \mathrm{abc}$ & $140.35 \mathrm{ab}$ & $154.4 \mathrm{abcd}$ & 147.38 abcd \\
\hline $\mathrm{T}_{10}$ & $\mathrm{~A}_{1} \mathrm{~B}_{1} \mathrm{C}_{1}$ & $92.08 \mathrm{a}$ & $101.1 \mathrm{ab}$ & $96.59 \mathrm{ab}$ & $116.28 \mathrm{ab}$ & $125.88 \mathrm{a}$ & $121.08 \mathrm{ab}$ & $154.43 \mathrm{a}$ & $166.55 \mathrm{ab}$ & $160.49 \mathrm{ab}$ \\
\hline $\mathrm{T}_{11}$ & $\mathrm{~A}_{1} \mathrm{~B}_{2} \mathrm{C}_{0}$ & $86.29 \mathrm{ab}$ & $87.81 \mathrm{abc}$ & $87.05 \mathrm{abc}$ & $102.86 \mathrm{bcd}$ & $115.46 \mathrm{a}$ & $109.16 \mathrm{abc}$ & $140.80 \mathrm{ab}$ & $154.39 \mathrm{abcd}$ & 147.60 abcd \\
\hline $\mathrm{T}_{12}$ & $\mathrm{~A}_{1} \mathrm{~B}_{2} \mathrm{C}_{1}$ & $93.09 \mathrm{a}$ & $101.31 \mathrm{a}$ & $97.20 \mathrm{a}$ & $122.56 \mathrm{a}$ & $130.36 \mathrm{a}$ & $126.46 \mathrm{a}$ & $158.74 \mathrm{a}$ & $173.94 \mathrm{a}$ & $166.34 \mathrm{a}$ \\
\hline $\mathrm{T}_{13}$ & $\mathrm{~A}_{2} \mathrm{~B}_{0} \mathrm{C}_{0}$ & $68.77 \mathrm{cde}$ & $82.82 \mathrm{bcde}$ & $75.80 \mathrm{~cd}$ & 88.25 defgh & 108.57 abcd & 98.41 cdef & 107.30 cdefg & 126.46 cdefgh & 116.88 cdefg \\
\hline $\mathrm{T}_{14}$ & $\mathrm{~A}_{2} \mathrm{~B}_{0} \mathrm{C}_{1}$ & $86.16 \mathrm{ab}$ & 84.84abcd & $85.50 \mathrm{abc}$ & 92.37 defgh & $110.96 \mathrm{ab}$ & $101.67 \mathrm{~cd}$ & 133.06 abcde & 150.79 abcde & 141.93 abcde \\
\hline $\mathrm{T}_{15}$ & $\mathrm{~A}_{2} \mathrm{~B}_{1} \mathrm{C}_{0}$ & $72.78 \mathrm{~cd}$ & $83.81 \mathrm{abcd}$ & $78.30 \mathrm{~cd}$ & 87.01 efghi & $111.32 \mathrm{ab}$ & 99.17 cde & 116.98 bcdefg & 147.87 abcdef & 132.43 abcdef \\
\hline $\mathrm{T}_{16}$ & $\mathrm{~A}_{2} \mathrm{~B}_{1} \mathrm{C}_{1}$ & $75.19 \mathrm{bcd}$ & 93.79abc & $84.49 \mathrm{abc}$ & 97.15 cdef & $117.8 \mathrm{a}$ & $107.48 \mathrm{bc}$ & 138.74 abcd & 156.94 abcd & 147.84 abcd \\
\hline $\mathrm{T}_{17}$ & $\mathrm{~A}_{2} \mathrm{~B}_{2} \mathrm{C}_{0}$ & $69.82 \mathrm{cde}$ & $82.53 \mathrm{cde}$ & $76.18 \mathrm{~cd}$ & 96.14 cdefg & 108.17 abcde & $102.16 \mathrm{c}$ & 126.11 abcdefg & 143.61 abcdefg & 134.86 abcdef \\
\hline $\mathrm{T}_{18}$ & $\mathrm{~A}_{2} \mathrm{~B}_{2} \mathrm{C}_{1}$ & $78.78 \mathrm{bc}$ & $96.00 \mathrm{abc}$ & $87.39 \mathrm{abc}$ & $111.65 \mathrm{abc}$ & $119.46 \mathrm{a}$ & $115.56 \mathrm{abc}$ & $140.97 \mathrm{ab}$ & $159.45 \mathrm{abc}$ & $150.21 \mathrm{abc}$ \\
\hline \multicolumn{2}{|c|}{ S.Em. $( \pm)$} & 4.32 & 6.43 & 4.88 & 5.41 & 9.28 & 6.52 & 11.61 & 13.10 & 12.17 \\
\hline \multicolumn{2}{|c|}{ L.S.D. $(\mathrm{P} \leq 0.05)$} & 12.41 & 18.49 & 14.01 & 15.55 & 26.67 & 18.75 & 33.35 & 37.64 & 34.97 \\
\hline
\end{tabular}

MAP-Month after planting

**Means with the same letter are not significantly different 
Table.7 Effect of Nutrient management on D-leaf area $\left(\mathrm{cm}^{2}\right)$ (contd....)

\begin{tabular}{|c|c|c|c|c|c|c|c|c|c|}
\hline \multirow[t]{2}{*}{ Treatments } & \multicolumn{3}{|c|}{12 MAP } & \multicolumn{3}{|c|}{15 MAP } & \multicolumn{3}{|c|}{18 MAP } \\
\hline & 2015 & 2016 & Mean & 2015 & 2016 & Mean & 2015 & 2016 & Mean \\
\hline $\mathrm{A}_{0}$ & $133.29 \mathrm{c}$ & $158.56 \mathrm{~b}$ & $145.93 b$ & $173.52 b$ & $186.07 \mathrm{~b}$ & $179.79 b$ & $216.33 b$ & $224.67 b$ & $220.50 \mathrm{~b}$ \\
\hline $\mathrm{A}_{1}$ & $202.30 \mathrm{a}$ & $206.51 \mathrm{a}$ & $204.4 \mathrm{a}$ & $234.35 \mathrm{a}$ & $240.60 \mathrm{a}$ & $237.48 \mathrm{a}$ & $280.48 \mathrm{a}$ & $293.04 a$ & $286.76 \mathrm{a}$ \\
\hline $\mathrm{A}_{2}$ & $189.23 b$ & $192.45 \mathrm{a}$ & $190.84 \mathrm{a}$ & $222.51 \mathrm{a}$ & $232.74 a$ & $227.63 \mathrm{a}$ & $266.01 \mathrm{a}$ & $280.03 \mathrm{a}$ & $273.32 \mathrm{a}$ \\
\hline S.Em. $( \pm)$ & 4.36 & 6.23 & 4.80 & 6.22 & 6.17 & 6.12 & 5.54 & 6.24 & 5.77 \\
\hline L.S.D(P $\leq \mathbf{0 . 0 5})$ & 12.53 & 17.92 & 13.79 & 17.87 & 17.73 & 17.60 & 15.92 & 17.95 & 16.58 \\
\hline $\mathrm{B}_{0}$ & $163.02 \mathrm{~b}$ & $173.65 b$ & $168.33 b$ & $201.16 \mathrm{~b}$ & $209.40 \mathrm{~b}$ & $205.28 \mathrm{~b}$ & $239.88 b$ & $250.68 \mathrm{~b}$ & $245.28 \mathrm{~b}$ \\
\hline $\mathrm{B}_{1}$ & $176.14 \mathrm{a}$ & $186.26 \mathrm{~b}$ & $181.2 \mathrm{ab}$ & $209.59 \mathrm{ab}$ & $220.03 b$ & $214.77 \mathrm{a}$ & $257.38 \mathrm{a}$ & $268.35 b$ & $262.87 \mathrm{a}$ \\
\hline $\mathrm{B}_{2}$ & $185.67 \mathrm{a}$ & $197.61 \mathrm{a}$ & $191.64 \mathrm{a}$ & $219.71 \mathrm{a}$ & $229.99 a$ & $224.85 \mathrm{a}$ & $266.17 \mathrm{a}$ & $278.72 \mathrm{a}$ & $272.44 a$ \\
\hline S.Em. $( \pm)$ & 4.36 & 6.23 & 4.80 & 6.22 & 6.17 & 6.12 & 5.54 & 6.24 & 5.72 \\
\hline L.S.D(P $\leq \mathbf{0 . 0 5})$ & 12.53 & 17.92 & 13.79 & 17.87 & 17.73 & 17.60 & 15.92 & 17.95 & 16.58 \\
\hline $\mathrm{C}_{0}$ & $163.70 \mathrm{~b}$ & $172.36 \mathrm{~b}$ & $168.03 \mathrm{~b}$ & $200.36 b$ & $210.15 b$ & $205.25 b$ & $239.84 b$ & $251.55 b$ & $245.69 \mathrm{~b}$ \\
\hline $\mathrm{C}_{1}$ & $186.18 \mathrm{a}$ & $199.32 \mathrm{a}$ & $192.75 \mathrm{a}$ & $219.90 \mathrm{a}$ & $229.46 a$ & $224.68 \mathrm{a}$ & $269.11 \mathrm{a}$ & $280.28 \mathrm{a}$ & $274.70 \mathrm{a}$ \\
\hline S.Em. ( \pm$)$ & 3.56 & 5.09 & 3.91 & 5.08 & 5.04 & 5.00 & 4.52 & 5.10 & 4.71 \\
\hline L.S.D(P $\leq$ 0.05) & 10.23 & 14.63 & 11.26 & 14.59 & 14.48 & 14.37 & 13.00 & 14.65 & 13.54 \\
\hline
\end{tabular}

\begin{tabular}{|c|c|c|c|c|c|c|c|c|c|c|}
\hline \multirow{2}{*}{\multicolumn{2}{|c|}{$\begin{array}{l}\text { Treatments/ } \\
\text { Combination }\end{array}$}} & \multicolumn{3}{|c|}{12 MAP } & \multicolumn{3}{|c|}{15 MAP } & \multicolumn{3}{|c|}{18 MAP } \\
\hline & & \multirow{2}{*}{$\begin{array}{r}\mathbf{2 0 1 5} \\
124.68 \mathrm{~h}\end{array}$} & \multirow{2}{*}{$\begin{array}{r}\mathbf{2 0 1 6} \\
136.19 \mathrm{~g} \\
\end{array}$} & \multirow{2}{*}{$\begin{array}{c}\text { Mean } \\
130.44 \mathrm{~h}\end{array}$} & \multirow{2}{*}{$\begin{array}{r}\mathbf{2 0 1 5} \\
154.44 \mathrm{f}\end{array}$} & \multirow{2}{*}{$\begin{array}{r}\mathbf{2 0 1 6} \\
164.57 \mathrm{f}\end{array}$} & \multirow{2}{*}{$\begin{array}{l}\text { Mean } \\
159.51 \mathrm{f}\end{array}$} & \multirow{2}{*}{$\begin{array}{r}\mathbf{2 0 1 5} \\
187.19 \mathrm{~h}\end{array}$} & \multirow{2}{*}{$\begin{array}{r}\mathbf{2 0 1 6} \\
192.21 \mathrm{f}\end{array}$} & \multirow{2}{*}{$\begin{array}{c}\text { Mean } \\
189.70 \mathrm{j}\end{array}$} \\
\hline $\mathrm{T}_{1}$ & $\mathrm{~A}_{0} \mathrm{~B}_{0} \mathrm{C}_{0}$ & & & & & & & & & \\
\hline $\mathrm{T}_{2}$ & $\mathrm{~A}_{0} \mathrm{~B}_{0} \mathrm{C}_{1}$ & $135.23 \mathrm{gh}$ & 163.23defg & $149.23 \mathrm{fgh}$ & 177.39def & 191.59def & 184.49def & 228.61efg & 234.51def & 231.56ghi \\
\hline $\mathrm{T}_{3}$ & $\mathrm{~A}_{0} \mathrm{~B}_{1} \mathrm{C}_{0}$ & $130.35 \mathrm{gh}$ & $145.81 \mathrm{fg}$ & $138.08 \mathrm{gh}$ & $164.29 \mathrm{f}$ & $174.11 \mathrm{f}$ & $169.20 \mathrm{f}$ & $205.73 \mathrm{gh}$ & $210.25 \mathrm{ef}$ & $207.99 \mathrm{j}$ \\
\hline $\mathrm{T}_{4}$ & $\mathrm{~A}_{0} \mathrm{~B}_{1} \mathrm{C}_{1}$ & $134.87 \mathrm{gh}$ & 163.37defg & $149.12 \mathrm{fgh}$ & $184.38 \mathrm{cdef}$ & 193.81cdef & 189.10cdef & 227.28efg & 234.96def & 231.12ghi \\
\hline $\mathrm{T}_{5}$ & $\mathrm{~A}_{0} \mathrm{~B}_{2} \mathrm{C}_{0}$ & $133.96 \mathrm{gh}$ & $154.37 \mathrm{efg}$ & 144.17fgh & $172.34 \mathrm{ef}$ & $186.18 \mathrm{ef}$ & $179.26 \mathrm{ef}$ & $216.71 \mathrm{fgh}$ & $223.4 \mathrm{ef}$ & $220.06 \mathrm{ghi}$ \\
\hline $\mathrm{T}_{6}$ & $\mathrm{~A}_{0} \mathrm{~B}_{2} \mathrm{C}_{1}$ & $140.67 \mathrm{gh}$ & $188.37 \mathrm{bcdefg}$ & $164.52 \mathrm{efg}$ & $188.26 \mathrm{cdef}$ & $206.15 \mathrm{cdef}$ & $197.21 \mathrm{cdef}$ & $232.47 \mathrm{efg}$ & $252.72 \mathrm{cde}$ & 242.60fghi \\
\hline $\mathrm{T}_{7}$ & $\mathrm{~A}_{1} \mathrm{~B}_{0} \mathrm{C}_{0}$ & 175.66def & 176.96cdefg & $176.31 \mathrm{de}$ & $216.17 \mathrm{bcd}$ & 223.63bcde & 219.90bcde & 247.00def & $268.31 \mathrm{bcd}$ & 257.66defg \\
\hline $\mathrm{T}_{8}$ & $\mathrm{~A}_{1} \mathrm{~B}_{0} \mathrm{C}_{1}$ & $191.82 \mathrm{bcde}$ & 196.03abcde & $193.93 \mathrm{bcde}$ & $224.35 \mathrm{abc}$ & $222.54 \mathrm{bcde}$ & $223.45 \mathrm{bcd}$ & $258.49 \mathrm{cde}$ & $271.23 \mathrm{bcd}$ & $264.86 \mathrm{cdefg}$ \\
\hline $\mathrm{T}_{9}$ & $\mathrm{~A}_{1} \mathrm{~B}_{1} \mathrm{C}_{0}$ & $192.53 \mathrm{bcde}$ & 201.31abcd & $196.92 \mathrm{bcde}$ & $221.95 b c$ & $236.22 \mathrm{abc}$ & $229.09 \mathrm{abc}$ & $272.05 \mathrm{bcd}$ & $287.98 \mathrm{abc}$ & 280.02 bcdef \\
\hline $\mathrm{T}_{10}$ & $\mathrm{~A}_{1} \mathrm{~B}_{1} \mathrm{C}_{1}$ & $221.83 \mathrm{ab}$ & $227.08 \mathrm{ab}$ & $224.46 \mathrm{ab}$ & $250.82 \mathrm{ab}$ & $255.24 \mathrm{ab}$ & $253.03 \mathrm{ab}$ & $301.17 \mathrm{ab}$ & $310.47 \mathrm{ab}$ & $305.82 \mathrm{ab}$ \\
\hline $\mathrm{T}_{11}$ & $\mathrm{~A}_{1} \mathrm{~B}_{2} \mathrm{C}_{0}$ & 197.95bcde & 202.34abcd & $200.15 \mathrm{bcd}$ & $226.14 \mathrm{abc}$ & $237.03 \mathrm{abc}$ & 231.59abc & $278.88 \mathrm{bcd}$ & $290.5 \mathrm{abc}$ & 284.69bcde \\
\hline $\mathrm{T}_{12}$ & $\mathrm{~A}_{1} \mathrm{~B}_{2} \mathrm{C}_{1}$ & $233.99 \mathrm{a}$ & $235.34 \mathrm{a}$ & $234.67 \mathrm{a}$ & $266.65 \mathrm{a}$ & $268.96 \mathrm{a}$ & $267.81 \mathrm{a}$ & $325.31 \mathrm{a}$ & $329.77 \mathrm{a}$ & $327.54 \mathrm{a}$ \\
\hline $\mathrm{T}_{13}$ & $\mathrm{~A}_{2} \mathrm{~B}_{0} \mathrm{C}_{0}$ & $157.68 \mathrm{fg}$ & 171.3defg & $164.49 \mathrm{efg}$ & 214.51bcde & 219.66bcde & 217.09bcde & 243.78defg & $249.57 \mathrm{cde}$ & 246.68efghi \\
\hline $\mathrm{T}_{14}$ & $\mathrm{~A}_{2} \mathrm{~B}_{0} \mathrm{C}_{1}$ & $193.02 \mathrm{bcde}$ & 198.19abcde & $195.61 \mathrm{bcd}$ & $220.11 \mathrm{bcd}$ & 234.39abcd & $227.25 \mathrm{abcd}$ & $274.21 \mathrm{bcd}$ & $288.23 a b c$ & 281.22 bcdef \\
\hline $\mathrm{T}_{15}$ & $\mathrm{~A}_{2} \mathrm{~B}_{1} \mathrm{C}_{0}$ & $171.47 \mathrm{ef}$ & 173.99cdefg & 172.73def & $214.70 \mathrm{bcde}$ & 225.14bcde & 219.92bcde & $255.75 \mathrm{cde}$ & $273.02 \mathrm{bcd}$ & 264.39cdefg \\
\hline $\mathrm{T}_{16}$ & $\mathrm{~A}_{2} \mathrm{~B}_{1} \mathrm{C}_{1}$ & 205.77abcd & 205.98abcd & 205.88abcd & $220.90 \mathrm{bcd}$ & $235.66 \mathrm{abc}$ & $228.28 \mathrm{abc}$ & $282.30 \mathrm{bcd}$ & $293.40 \mathrm{abc}$ & $287.85 \mathrm{abcd}$ \\
\hline $\mathrm{T}_{17}$ & $\mathrm{~A}_{2} \mathrm{~B}_{2} \mathrm{C}_{0}$ & $188.98 \mathrm{cde}$ & 188.94bcdef & $188.96 \mathrm{cde}$ & $218.68 \mathrm{bcd}$ & $224.77 \mathrm{bcde}$ & 221.73bcde & 251.48def & $268.69 \mathrm{bcd}$ & 260.09cdefgh \\
\hline $\mathrm{T}_{18}$ & $\mathrm{~A}_{2} \mathrm{~B}_{2} \mathrm{C}_{1}$ & $218.44 \mathrm{abc}$ & $216.31 \mathrm{abc}$ & $217.38 \mathrm{abc}$ & $246.19 \mathrm{ab}$ & $256.83 \mathrm{ab}$ & $251.51 \mathrm{ab}$ & $292.16 a b c$ & $307.25 \mathrm{ab}$ & 299.71abc \\
\hline \multicolumn{2}{|c|}{ S.Em. $( \pm)$} & 10.68 & 15.27 & 11.75 & 15.23 & 15.11 & 15.00 & 13.57 & 15.29 & 14.13 \\
\hline \multicolumn{2}{|c|}{ L.S.D. $(\mathrm{P} \leq 0.05)$} & 30.68 & 43.89 & 33.77 & 43.77 & 43.42 & 43.11 & 39.00 & 43.96 & 40.62 \\
\hline
\end{tabular}
MAP-Month after planting

**Means with the same letter are not significantly different 
It is clear from the tables 5, 6 and 7 that the D-leaf length, breadth and area has increased continuously from 3 months after planting to eighteen months after planting in both 2015, 2016 and significantly varied among the most of the treatments for both the years and for pooled mean values which suggest there is a role of integrated nutrient management for growth behaviour of pineapple. It is also noticed that organic manure as main factor has non-significant role for D-leaf length, breadth and leaf area for some observation time. The rate of increase of length was higher from 9 to 12 and 12 to 15 months after planting. At 18 months after planting the D-leaf length (pooled) was highest $(63.35 \mathrm{~cm})$ with $\mathrm{T}_{12}$ which was statistically at par with $\mathrm{T}_{10}$ and $\mathrm{T}_{18}$. Almost similar observation was recorded (pooled) for $\mathrm{D}$ leaf breadth which was recorded maximum $(5.17 \mathrm{~cm})$ with $\mathrm{T}_{12}$. It is also observed from the pooled means of table 7 that the highest D-leaf area $\left(327.54 \mathrm{~cm}^{2}\right)$ was recorded with $\mathrm{T}_{12}$ which was statistically at par with $\mathrm{T}_{10}\left(305.82 \mathrm{~cm}^{2}\right), \mathrm{T}_{18}$ $\left(299.71 \mathrm{~cm}^{2}\right)$, and $\mathrm{T}_{16} \quad\left(287.85 \mathrm{~cm}^{2}\right)$. Several scientist had reported regarding the nutritive management which confirms the result of present experiment. Omotoso and Akinrinde (2013) and (Bhugaloo, 1998). Reported the effect of $\mathrm{N}$ fertilizer application on growth and behavior in pineapple. Singh et al., (2010), to study the response of integrated nutrient management on growth, yield and quality of papaya cv. Surya. Organic manure, urea and their combination have also important role in growth behavior like D-leaf length, number of leaves, root length and leaf area (Omotoso and Akinrinde, 2012).

The different parameters for growth behaviour study of pineapple cv. Mauritius under the integrated nutrient management showed significant variation for 2015, 2016 and pooled means among most of the treatments. It is also found from the present experiment that the treatment combinations having chemical fertilizer, organic manure and bio-fertilizers shows better performance than the other treatment combinations. The plant height, canopy spread, number of leaves, D-leaf length, D-leaf breadth, and leaf area was recorded highest in $\mathrm{T}_{12}$ $\left(\mathrm{A}_{1} \mathrm{~B}_{2} \mathrm{C}_{1}\right)$.

\section{References}

Anonymous, 2017. Accessed from on http://prsvkm.kau.in/ sites/ default/ files/ documents/pineapple_sector_in_kerala_stat us_opportunities_challenges_and_stakehold ers. pdf on 12.6.2016

Bhugaloo, R. A., 1998. Effect of different levels of nitrogen on yield and quality of pineapple variety Queen Victoris. Food and Agricultural Research Council, Reduit, Mauritius.

Jacob, C., and Soman M. 2006. In: Pineapples. Working Paper Series. Institute for Financial Management and Research Centre for Development Finance.

Omotoso, S.O., and Akinrinde E. A. 2013. Effect of nitrogen fertilizer on some growth, yield and fruit quality parameters in pineapple (Ananas comosus L. Merr.) plant at AdoEkiti Southwestern, Nigeria. International Research Journal of Agricultural Science and Soil Science, 3(1): 11-16

Omotoso, S.O., and Akinrinde, E. A. 2012. Effects of nutrient sources on the early growth of pineapple plantlets (Ananas comosus (L) Merr) in the nursery. Journal of Fruit and Ornamental Plant Research, 20(2): $35-40$

Reddy, B.M.C., and Prakash, G. S. 1982. Standardization of optimum depth of trench of planting Kew Pineapple. In: Annual Report. Indian Institute of Horticultural Research, p. 19.

Singh, K., Barche, K. S., and Singh, D. B. 2010. Integrated nutrient management in papaya (Carica papaya) cv. Surya. Acta Horticulturae, 851:377-380

\section{How to cite this article:}

Nilesh Bhowmick, Partha Sarathi Munsi, Swapan Kumar Ghosh, Prahlad Deb and Arunava Ghosh. 2017. Growth Behavior of Pineapple cv. Mauritius under Integrated Nutrient Management in Northern part of West Bengal, India. Int.J.Curr.Microbiol.App.Sci. 6(9): 2471-2488.

doi: https://doi.org/10.20546/ijcmas.2017.609.305 